Project No. 09-809

\title{
Simulations of the Thermodynamic and Diffusion Properties of Actinide Oxide Fuel Materials
}

Fuel Cycle R\&D

Dr. Udo Becker

University of Michigan

Sue Lesica, Federal POC Ken McClellan, Technical POC

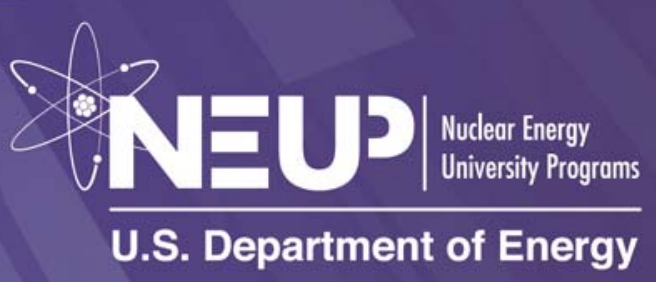




\section{NEUP end of grant report}

\section{Short summary of achievements}

NEUP grant DE-AC07-05ID14517 concluded on 08/31/12. The objectives of this grant were to (1) determine the solid solution thermodynamics of actinide dioxides and (2) evaluate actinide diffusion in $\mathrm{UO}_{2}$. During the three-year grant period, we published six peer-reviewed articles, two peer-reviewed conference proceedings, and disseminated eleven presentations. The papers are attached at the end of this report. In both major objectives and in all sub-objectives of these, we have succeeded to do the necessary method development and derived the thermodynamic mixing properties of a number of fuel-relevant solid solutions and the associated phase diagrams for objective 1 . In addition, we developed the basis for actinide diffusion involving different vacancy mechanism and for interstitial He diffusion in fuels and potential waste forms.

\subsection{Solid Solutions}

The major advances in the method development for deriving the mixing thermodynamics of solid solutions were done by modeling different oxide solutions, not only with different compositions but also using different structure types (up to three in the same solid solution series).

A fairly standardized approach was used to first develop cation-cation interaction parameters from quantum-mechanical energies of small configuration arrangements with subsequent Monte Carlo calculations of large systems. Further thermodynamic integration allows for the study of temperature effects on the free energy and entropy of mixing of the solid solutions. In the framework of this objective, we analyzed the results beyond what has been done before. We were able to evaluate the apparent discrepancy in the temperature of exsolution onset from the Gibbs free energy of mixing diagrams and the evidence from the projection of the cation ordering. By measuring the height of the Gibbs free energy peak with respect to the tangent of the curve, we were able to determine the exsolution driving force, which was further compared to the kinetic hindrances to exsolution. The primary hindrance to exsolution is the surface energy built up between exsolved lamellae. While the final deliverable was a temperature-composition phase diagram (as described in the proposal), we went beyond by analyzing the effects of cation ordering based on different cation-cation interaction parameter fit models (i.e., fitting with and without a configuration independent energy term). The different fit options allow the Monte Carlo simulations to more closely model an exsolving system. Thus, by comparing the two different approaches, the two extremes - disorder and exsolution - could be simulated.

\section{Implications}

$\mathrm{Th}_{x} U_{1-x} \mathrm{O}_{2}$ solid solution: this is the first atomic-scale investigation of the energetics and cation ordering in the $\mathrm{Th}_{\mathrm{x}} \mathrm{U}_{1-\mathrm{x}} \mathrm{O}_{2}$ system has not been conducted. However, according to the computational analysis exsolution temperatures are so low that exsolution in natural uranothorianite and synthetic ThxU1_xO2 is not expected. This has potential implications for fuel properties of the thorium fuel cycle as it has become evident that for typical reactor conditions, thorium and uranium dioxide solid solutions would not exsolve.

$(\mathrm{Zr}, \mathrm{Th}, \mathrm{Ce}) \mathrm{O}_{2}$ solid solutions: Mixed-Th-U fuels are under active investigation [1] and have been tested in power reactors (e.g., Shippingport and Fort St. Vrain in the United States and 
KAPS in India [2]). For such mixed Th-U fuels, $\mathrm{Zr}$ and Ce may be present as part of the initial fuel matrix (e.g., inert matrix fuel) or part of the final fuel matrix as fission products. As determined experimentally, the Th-Ce binary forms a complete solid solution, and no cation ordering or nanoscale exsolution was observed. Below room temperature at $x^{1 / 40.5}$, nanoscale exsolution with lamellae 99 to $\partial 102 \mathrm{P}$ are observed for the $\mathrm{Ce}_{\mathrm{x}} \mathrm{Zr}_{1-\mathrm{x}} \mathrm{O}_{2}$ binary. At the same conditions, a complex cation-ordering scheme is observed for the $\mathrm{Th}-\mathrm{Zr}$ binary. Exsolution is expected for the solid solutions containing $\mathrm{Zr}$ based on the large miscibility gap observed in the Gibbs free energy of mixing, as well as the instability of isometric and tetragonal $\mathrm{ZrO}_{2}$ at room temperature. However, $\mathrm{ZrO}_{2}$ does have limited solubility for both $\mathrm{CeO}_{2}$ and $\mathrm{ThO}_{2}$. The discrepancy between measured and calculated estimates of miscibility may be reduced by further calculations in which the $\mathrm{ZrO}_{2}$ end-member is considered to be monoclinic ( $\left.\mathrm{P} 2_{1} / \mathrm{c}\right)$, which is the most stable form at room temperature. Further analysis of the complete range of $\mathrm{Ce}_{\mathrm{x}} \mathrm{Zr}_{1-\mathrm{x}} \mathrm{O}_{2}$ and $\mathrm{Th}_{\mathrm{x}} \mathrm{Zr}_{1-\mathrm{x}} \mathrm{O}_{2}$ solid solutions within a monoclinic framework is necessary in order to understand the impact of the structural stability on the solid-solution properties of these binaries.

$U_{x} H_{1-x} \mathrm{O}_{2}$ solid solution: Neutron absorbers are used in fuel rods in order to depress the power level of freshly loaded nuclear fuel $\mathrm{UO}_{2}$ and to permit higher loading of fuel to achieve longer core life. Neutron poison materials can be coated surrounding fuel pellets, doped into the cladding materials or mixed with $\mathrm{UO}_{2}$. Incorporation of burnable neutron poison materials into the fuel can level the power distribution such that power is generated across the core region rather than produced only close to the small area where control rods have been removed. Our calculations show that close to the $\mathrm{UO}_{2}$ end member, which is relevant to the nuclear fuel, uranium-rich solid solutions exsolve as the fuel cools, and there is a tendency to form the monoclinic hafnium-rich phase in the matrix of isometric uranium-rich solid solution. Close to the compositional range of the $\mathrm{UO}_{2}$ end member, uranium-rich solid solutions exsolve as the temperature decrease into the miscibility gap which may have implications on the overall performance of the fuel. The calculated phase diagram indicates a tendency to form the monoclinic hafnium-rich phase in the matrix of isometric uranium-rich solid-solution as the fuel cools. Cubic to monoclinic phase transitions of the $\mathrm{UO}_{2}-\mathrm{HfO}_{2}$ binary also involves about 7-10\% increase in the cell volume.

\subsection{Diffusion}

Diffusion is related to the study of thermodynamics in that once a homogeneous mixture is not stable any more (miscibility gap in solid solution, fission product production, noble gas production ..), a kinetic process can kick in that attempts to separate phases, e.g., in the fuel, the used fuel, or the waste form. The kinetics of diffusion is mainly governed by the attempt frequency of a species to jump from one energetic minimum to the next and by the temperature dependent (Boltzmann) probability for a successful hop.

Depending on the matrix, we looked at three very different diffusion mechanisms:

1. Diffusion of interstitial oxygen atoms in hyperstoichiometric uranium dioxide.

2. Diffusion of actinides in uranium dioxide by a coupled mechanism that includes the simultaneous formation of different vacancy types.

3. Diffusion of noble gases in potential waste forms such as apatite.

Each of these mechanisms required a significant amount of model development, as described below, and an even greater amount of computational resources. However, we believe that we 
made significant progress in each of the three fields.

\section{Implications}

Noble gas diffusion in apatite and zircon: He diffusion in ideal zircon is greater than in ideal apatite and anisotropic in both. However, the degree of anisotropy is much more pronounced in zircon. The computational approach allows a comparison of the behavior of the ideal structures (i.e., defect-free) as compared to natural samples that may contain impurities or some level of radiation damage. Under most conditions, apatite will retain less radiation damage than zircon.

Diffusion of actinide ions in actinide oxides: Stabilities of actinide oxide solid solutions will affect their properties, e.g., chemical and mechanical stabilities, relevant to reprocessing or geologic disposal of the used nuclear fuel. Therefore, it is important to know whether phase separation or phase aggregation of the different oxides of these solid solutions will form and to what extent the ordered phases will form in these solid solutions. If a solid solution is not thermodynamically favorable, the oxide may or may not form separate phases, which is largely controlled by the kinetics of the phase separation or exsolution process. In this process, actinide diffusion in uranium dioxide plays an essential role in determining the kinetics whether phase separation actually occurs in case the thermodynamics is favorable for such a separation. In addition, diffusion of actinides in $\mathrm{UO}_{2}$ fuel also plays a role in determining the kinetics of the microstructure evolution such as structural deformation and void formation of the fuel at operation conditions in nuclear reactors. Fission gas diffusion and associated gas bubble formation in $\mathrm{UO}_{2}$ have been shown to be largely coupled or controlled by diffusion of $\mathrm{U}$ atoms in the structure. The calculated activation energies and their components of different actinides in $\mathrm{UO}_{2}$ have been calculated and the migration energies for Th, $\mathrm{U}, \mathrm{Np}$, and $\mathrm{Pu}$ are 5.4, 5.2, 4.9, and $4.0 \mathrm{eV}$, respectively. For Th diffusion in $\mathrm{UO}_{2}$, the calculated activation energy, $7.7 \mathrm{eV}$, is higher than the experimental value of $\_6.5 \mathrm{eV}$ of Th in $\mathrm{ThO}_{2}$. For $\mathrm{U}$ diffusion in $\mathrm{UO}_{2}$, the calculated value of $7.3 \mathrm{eV}$ is near the high end of the experimental values (i.e., 2.6-7.8 eV) using nonirradiated samples of single crystal uranium dioxide. 


\section{Results in more detail}

\subsection{Thermodynamic properties of actinide-oxide solid solutions}

We have focussed our calculations of thermodynamic properties of actinide-oxide fuels on two phenomena:

- $\quad$ Systematically determine the thermodynamic properties of actinide oxide solid solutions. Specifically, calculate the temperature-dependent excess enthalpy of mixing, excess free energy of mixing, and excess configurational entropy.

- $\quad$ From the excess free energies, phase diagrams can be derived for any composition and temperature; this allows the identification of miscibility gaps and possible compositional ordering for each solid-solution binary (ternary, quaternary).

\section{Thermodynamics of oxide solid solutions}

The major advances in computational methodology in terms of the analysis of solid solution calculations were in the field of the excess thermodynamic properties of different oxide solutions. We used a well-researched method to fit cation-cation interaction parameters with quantum-mechanical energies such that our 24 cation system could be scaled up to 2048 cations in Monte Carlo simulations. Further thermodynamic integration allows for the study of temperature effects on the free energy and entropy of mixing of the solid solutions. In this body of work, we analyzed the results beyond what has been done prior. We were able to evaluate the apparent discrepancy in the temperature of exsolution onset from the Gibbs free energy of mixing diagrams and the evidence from the projection of the cation ordering. By measuring the height of the Gibbs free energy peak with respect to the tangent of the curve, we were able to determine the exsolution driving force, which was further compared to the hindrances to exsolution. The primary hindrance to exsolution is the surface energy built up between exsolved lamellae. While the final deliverable was a temperature-composition phase diagram, we further analyzed effects of cation ordering based on different cation-cation interaction parameter fit models (i.e., fitting with and without a Margules parameter). The different fit options allow the Monte Carlo simulations to more closely model an exsolved system. Thus, by comparing the fits, we were able to simulate scenarios at two extremes - disorder and exsolution.

1.4. Thermodynamic properties of $\mathrm{Th}_{\mathrm{x}} \mathrm{U}_{1} \mathrm{xO}_{2}(0<\mathrm{x}<1)$ based on quantummechanical calculations and Monte-Carlo simulations

$\mathrm{Th}_{\mathrm{X}} \mathrm{U}_{\mathrm{I}_{-} \mathrm{O}} \mathrm{O}_{2+\mathrm{y}}$ binary compositions occur in nature, uranothorianite, and as a mixed oxide nuclear fuel. As a nuclear fuel, important properties, such as the melting point, thermal conductivity, and the thermal expansion coefficient change as a function of composition. Additionally, for direct disposal of $\mathrm{Th}_{x} \mathrm{U}_{1 \_} \mathrm{O}_{2}$, the chemical durability changes as a function of composition, with the dissolution rate decreasing with increasing thoria content. $\mathrm{UO}_{2}$ and $\mathrm{ThO}_{2}$ have the same isometric structure, and the ionic radii of 8-fold coordinated $\mathrm{U}_{4^{+}}$and $\mathrm{Th}_{4_{+}}$are similar $(1.14 \mathrm{~nm}$ and $1.19 \mathrm{~nm}$, respectively). Thus, this binary is expected to form a complete solid solution. However, atomicscale measurements or simulations of cation ordering and the associated thermodynamic properties of the $\mathrm{Th}_{\times} \mathrm{U}_{1_{-} \times} \mathrm{O}_{2}$ system have yet to be determined. A combination of density-functional 
theory, Monte-Carlo methods, and thermodynamic integration are used to calculate thermodynamic properties of the $T h_{x} \mathrm{U}_{1_{-} \times} \mathrm{O}_{2}$ binary $\left(\Delta \mathrm{H}_{\text {mix }}, \Delta \mathrm{G}_{\text {mix }}, \Delta \mathrm{S}_{\text {mix }}\right.$, phase diagram). The Gibbs free energy of mixing ( ${ }^{D} G_{\text {mix }}$ ) shows a miscibility gap at equilibration temperatures below $1000 \mathrm{~K}$ (e.g., $\mathrm{E}_{\text {exsoln }}=0.13 \mathrm{~kJ} /(\mathrm{mol}$ cations) at $750 \mathrm{~K}$ ). Such a miscibility gap may indicate possible exsolution (i.e., phase separation upon cooling). A unique approach to evaluate the likelihood and kinetics of forming interfaces between U-rich and Th-rich has been chosen that compares the energy gain of forming separate phases with estimated energy losses of forming necessary interfaces. The result of such an approach is that the thermodynamic gain of phase separation does not overcome the increase in interface energy between exsolution lamellae for thin exsolution lamellae (10 $\AA$ ). Lamella formation becomes energetically favorable with a reduction of the interface area and, thus, an increase in lamella thickness to $>45 \AA$. However, this increase in lamellae thickness may be diffusion limited. Monte-Carlo simulations converge to an exsolved structure [lamellae \| (22-1)] only for very low equilibration temperatures (below room temperature). In addition to the weak tendency to exsolve, there is an ordered arrangement of Th and $U$ in the solid solution [alternating $U$ and Th layers $\|\left\{\begin{array}{lll}1 & 0 & 0\end{array}\right]$ ] that is energetically favored for the homogeneously mixed 50\% Th configurations. Still, this tendency to order is so weak that ordering is seldom reached due to kinetic hindrances. The configurational entropy of mixing $\left(\Delta S_{\text {mix }}\right)$ is approximately equal to the point entropy at all temperatures, indicating that the system is not ordered.

\subsection{Atomistic calculations of the thermodynamic properties of mixing for tetravalent metal dioxide solid solutions: $(\mathrm{Zr}$, Th, Ce)O2}

The thermodynamic mixing properties for isometric $\mathrm{Th}_{\mathrm{x}} \mathrm{Ce}_{1 \_\mathrm{x}} \mathrm{O}_{2}, \mathrm{Ce}_{\mathrm{x}} \mathrm{Zr}_{1_{-} \mathrm{x}} \mathrm{O}_{2}$, and $\mathrm{Th}_{\mathrm{x}} \mathrm{Zr}_{1_{\_} \mathrm{x}} \mathrm{O}_{2}$ were determined using quantum-mechanical calculations and subsequent Monte-Carlo simulations. Although the $\mathrm{Th}_{\mathrm{x}} \mathrm{Ce}_{1_{-} \mathrm{x}} \mathrm{O}_{2}$ binary indicates exsolution below $600 \mathrm{~K}$, the energy gain due to exsolution is small ( $\mathrm{E}_{\text {exsoln }}=1.5 \mathrm{~kJ} /(\mathrm{mol}$ cations) at $200 \mathrm{~K})$. The energy gain for exsolution is significant for the binaries containing $\mathrm{Zr}$; at $1000 \mathrm{~K}, \mathrm{E}_{\text {exsoln }}=6 \mathrm{~kJ} /(\mathrm{mol}$ cations) for the $\mathrm{Ce}_{\mathrm{x}} \mathrm{Zr}_{1}{ }_{1} \mathrm{O} \mathrm{O}_{2}$ binary, and $\mathrm{E}_{\text {exsoln }}=17 \mathrm{~kJ} /\left(\mathrm{mol}\right.$ cations) for the $\mathrm{Th}_{\mathrm{x}} \mathrm{Zr}_{1 \_\mathrm{x}} \mathrm{O}_{2}$ binary. The binaries containing $\mathrm{Zr}$ have limited miscibility and cation ordering (at $200 \mathrm{~K}$ for $\mathrm{x}=0.5$ ). At $1673 \mathrm{~K}$, only 4.0 and $0.25 \mathrm{~mol} \% \mathrm{ZrO}_{2}$ can be incorporated into $\mathrm{CeO}_{2}$ and $\mathrm{ThO}_{2}$, respectively. Solidsolution calculations for the tetragonal $\mathrm{Th}_{\mathrm{x}} \mathrm{Zr}_{1 \_\mathrm{x}} \mathrm{O}_{2}$ binary show decreased mixing enthalpy due to the increased end-member stability of tetragonal $\mathrm{ZrO}_{2}$. Inclusion of the monoclinic $\mathrm{ZrO}_{2}$ is predicted to further reduce the mixing enthalpy for binaries containing $\mathrm{Zr}$.

\subsection{Density functional theory and Monte-Carlo simulations of the thermodynamic mixing properties of the $\mathrm{UO}_{2}-\mathrm{HfO}_{2}$ solid solution}

${ }^{177} \mathrm{Hf}$ is used as burnable neutron poison in nuclear fuel $\mathrm{UO}_{2}$. Quantum-mechanical calculations were applied to cubic, tetragonal and monoclinic $\mathrm{UO}_{2}$ and $\mathrm{HfO}_{2}$ supercells using density functional theory by two sets of parameters. Cation exchange parameters were then fit using the excess energy of mixing of different compositions, and the enthalpy of mixing was calculated using Monte Carlo simulations. Subsequent thermodynamic integration was performed to derive the Gibbs free energy of mixing. The results show that the $\mathrm{UO}_{2}-\mathrm{HfO}_{2}$ system calculated in a relative large cell forms extensive solid solution across the entire compositional range. However, in another set of calculations which leads to a smaller cell, wide range of unmixing region is found at low temperature. By comparison with stable phase region of the experimental determined $\mathrm{UO}_{2}-\mathrm{ZrO}_{2}$ phase diagram, the phase diagram of $\mathrm{UO}_{2}-\mathrm{HfO}_{2}$ with 
considerations of phase transition was obtained. At temperature below 1400K, umixing or cubicmonoclinic coexisted phases regions could occur. The expected region of the miscibility gap containing cubic-tetragonal phases is close to the end member of $\mathrm{UO}_{2}$ side. Above 2600K, complete solid solution with cubic structure is likely to be found.

\section{Diffusion}

The objectives in the diffusion section of this project as stated in the proposal were ill be performed to study the mechanism and kinetics of, e.g., phase separation and the formation of gas inclusions. Thus, the proposed diffusion calculations in actinide-oxide fuels were focussed on two phenomena:

- In the first part of the proposal, we have determined the thermodynamic properties of actinide oxide solid solutions, including burnable poisons. For some of the binaries/ternaries, there will be cation ordering, at least at the nano-scale; for others, exsolution may occur. For both processes, diffusion is necessary to transport the cation from its original position to the position in an exsolved phase. If diffusion is hindered, the more or less homogeneously disordered and, thus, thermodynamically less stable phase is the one that needs to be evaluated for the stability of a certain composition at a given temperature (rather than the most stable ordered or exsolved phase). Thus, diffusion mechanisms and constants will be derived as a function of temperature. As a result, the kinetics of phase separation has to be determined. This diffusion process is mainly a cation-cation swap process; in some cases, it may be mediated by the occurrence of defects in the structure.

- In addition to diffusion that leads to exsolution and ordering of the oxide matrix, the diffusion of fission product gases (I, Kr, Xe) needs to be evaluated. Fission product gases can weaken the oxide structure, act as neutron-capturing elements, and may outgas as a function of temperature. The diffusion mechanism of these gases is mainly interstitial.

\subsection{Attempt frequency of interstitial oxygen diffusion in hyperstoichiometric $\mathrm{UO}_{2+x}$}

Diffusion of interstitial oxygen atoms in hyperstoichiometric uranium dioxide plays important roles in a range of chemical and physical properties, such as thermal conductivity and corrosion of uranium dioxide. These properties are related to the performance of uranium dioxide nuclear fuel in nuclear reactors and safe disposal of used nuclear fuel.

In order to determine the diffusion coefficient of oxygen interstitial using first-principles calculations, the jump frequency for the oxygen migration were carried out for the diffusing atom by calculating the related partial Hessian matrix. The attempt frequency is calculated based on the equation:

$$
v=\frac{\prod_{i=1}^{3 N} v_{i}}{\prod_{i=1}^{3 N-1} v_{i}^{*}}
$$


Where, $v i=$ normal mode vibrational frequencies for the atom at the stable ground state and $\mathrm{vi}^{*}=$ normal mode vibrational frequencies for the atom at the activated state. Two frequency calculations were performed: one at the stable state, an oxygen interstitial was added in the octahedral vacant site; and one at the transition state, one oxygen was added in the middle of the two neighboring octahedral vacant sites. For the stable state calculation, the system was optimized before the frequency calculation. For the transition state calculation, the atom at the transition state was optimized in the plane perpendicular to the reaction/diffusion path before the frequency calculation. For the stable state, there are three frequencies: 391.8, 392.1, and 392.4 $\mathrm{cm}-1$, and for the transition state, there are two frequencies: 458.7 and $512.8 \mathrm{~cm}-1$. Thus, the attempt frequency is $256.2 \mathrm{~cm}-1$, or $\sim 0.8 \times 1013 \mathrm{~s}-1$. Another way to estimate the attempt frequency is to assume that the potential function of the diffusing atom is represented by a sinusoidal function and the frequency is then determined by the barrier height and jump distance. The results show that the calculated values based on the latter method is higher than the direct frequency calculation, suggesting that the sinusoidal potential overestimates the attempt frequency.

The results suggest that the direct frequency calculation used in this study is a better way to calculate the attempt frequency calculation in diffusion calculations, and should be used to the future attempt frequency calculations of atom diffusion.

\subsection{Activation energetics of actinide impurity diffusion in $\mathrm{UO}_{2}$}

Diffusion of actinides in uranium dioxide plays an important role in determining thermodynamic and mechanic properties of the material.

In this study, activation energies of $\mathrm{Th}, \mathrm{U}, \mathrm{Np}$, and $\mathrm{Pu}$ diffusion in uranium dioxide were systematically studied using first-principles calculations. The generalized gradient approximation and projector-augmented wave methods with on-site Coulomb repulsive interaction were applied within Density Functional Theory and Plane Wave framework. We have examined two diffusion paths, one along the lattice $<110>$ direction and the other along the lattice $<100>$ direction, in the face-centered cubic UO2 structure. The results show that the $<110>$ path has lower migration energy than the $<100>$ path. Under the assumption of a vacancy-assisted jump diffusion mechanism, we have calculated:

(i) Vacancy and binding energies, and

(ii) NEB calculations of migration barriers.

Our calculations show that the major contribution to the activation energy is the migration energy, followed by the vacancy formation energy and vacancy binding energy, where the last has the lowest contribution. However, differences in the activation energies among different actinides stem from both the migration and vacancy binding energies, both of which decrease with atomic number.

Our results show that there is an approximately linear relation between activation energy and atomic number and an asymptotic relation between activation energy and ionic radius of the actinides. The present study suggests that the migration of the actinides through the uranium dioxide lattice is closely correlated to the number of $5 \mathrm{f}$ electrons and the size of the diffusion atoms. We also aware that the discrepancies between the absolute values of the calculated and experimentally observed activation energies still remain, which calls for future investigation of 
actinide diffusion in the material.

\section{Attempt frequency of actinide impurity diffusion in $\mathrm{UO}_{2}$}

This is the second part of a study of actinide impurity diffusion in uranium dioxide. In this work, we focused on the attempt frequency calculation of the actinide atoms (Th, U, Np, and $\mathrm{Pu}$ ) in UO2.

The jump frequency for the oxygen migration were carried out for the diffusing atom by calculating the related partial Hessian matrix. The attempt frequency is calculated based on the equation:

$$
v=\frac{\prod_{i=1}^{3 N} v_{i}}{\prod_{i=1}^{3 N-1} v_{i}^{*}}
$$

Where, $v_{i}=$ normal mode vibrational frequencies for the atom at the stable ground state and $v_{i}^{*}=$ normal mode vibrational frequencies for the atom at the activated state. Two frequency calculations were performed: one at the stable state; and one at the transition state. Both of the states were carefully calculated using the Nudged Elastic Band (NEB) method using VASP.

The results are now being processed. The goal of this study is to demonstrate the capability of using first-principles methods to directly calculated self-diffusion coefficient of actinide diffusion in uranium dioxide, to resolve inconsistencies observed diffusion coefficients in the literature, and to reconcile the proposed cationic diffusion mechanisms using theoretical approaches.

\subsection{He diffusion in zircon and apatite as an example of uranium-induced interstitial diffusion}

Diffusion of He in zircon and apatite, as generated from the $\alpha$-decay of uranium in natural systems, is of fundamental importance in the interpretation of He-loss measurements used in thermochronology. In addition, it serves a s a model system for interstitial diffusion in solid materials. The diffusion of $\mathrm{He}$ in zircon is strongly anisotropic, while experimental measurements find He diffusion in apatite to be nearly isotropic. We present the first calculations for He diffusion in Ca-5(PO4)(3)F fluroapatite and re-calculate He diffusivity in zircon, ZrSiO4, in order to make a consistent comparison with the results of the apatite calculations and clarify discrepancies in the literature.

He diffusion in ideal zircon is greater than in ideal apatite and anisotropic in both. However, the degree of anisotropy is much more pronounced in zircon. The computational approach allows a comparison of the behavior of the ideal structures (i.e., defect-free) as compared to natural samples that may contain impurities or some level of radiation damage. The calculated diffusivities for the ideal structure are in closer agreement with experimentally determined values for natural apatite than for zircon. The calculations predict that the perfect zircon structure will have high diffusivities due to large uninterrupted "channels" along [001]. However, in natural samples, these channels may be interrupted due to the presence of impurities, e. g., radiogenic $\mathrm{Pb}$, or nanoscale radiation-damage cascades, $5 \mathrm{~nm}$ in diameter, created by the alpha-decay of incorporated $U$ and Th, thus effectively lowering the diffusivity. The damage microstructure 
depends on the fluence and thermal history of the sample, and variations in thermal history can lead to variations in the He-loss and the interpreted age and thermal history. Closure temperatures in the ideal structure are extremely low, -35 degrees $C$ for apatite and -150 degrees $\mathrm{C}$ for zircon, suggesting the degree of radiation damage plays an important role in attaining closure to He loss.

\section{Publications (peer-reviewed publications are attached)}

\subsection{Peer-reviewed publications}

\section{Solid solutions}

Shuller-Nickles, L.C., Ewing, R.C., and Becker, U. "Atomistic calculations of the thermodynamic properties of mixing for tetravalent metal dioxide solid solutions: (Zr,Ce,Th)O $\mathrm{O}_{2}$," Journal of Solid State Chemistry, 197, 550-559 (2012).

Shuller, L.C., Ewing, R.C., and Becker, U. "Thermodynamic properties of ThxU1-xO2 $(0<\mathrm{x}<$ 1) based on quantum-mechanical calculations and Monte-Carlo simulations," Journal of Nuclear Materials, 412, 13-21 (2011).

Ke Yuan K., Becker U., Ewing R.C. (submitted) Density functional theory and Monte-Carlo simulations of the thermodynamic mixing properties of the $\mathrm{UO}_{2}-\mathrm{HfO}_{2}$ solid solution, Journal of Nuclear Materials.

\section{Diffusion}

Wang, J., Becker, U., and Ewing, R.C. (2010) Electronic structure and the energetics of actinide diffusion in UO2. Flutonium Futures - The Science 2010, p354-355.

Wang, J. and Becker, U. (2012) Activation energetics of actinide diffusion in $\mathrm{UO}_{2}$ from firstprinciples calculations. (Accepted, Journal of Nuclear Materials, http://dx.doi.org/10.1016/j.jnucmat.2012.10.011).

Bengtson A., Ewing R.C., Becker U. (2012) He diffusion and closure temperatures in apatite and zircon: A density functional theory investigation. Geochimica et Cosmochimica Acta, 86, 228-238.

\subsection{Peer-reviewed Proceedings}

Shuller, L.C., Pavenayotin, N., Ewing, R.C., and Becker, U. "Thermodynamic properties of actinide dioxide solid solutions,” Materials Research Society Conference Proceedings, Materials Research Society, Boston, MA (November, 2008).

Shuller, L.C., Pavenayotin, N., Skomurski, F.N., Ewing, R.C., and Becker, U. "Thermodynamic properties of actinide-zirconium dioxide solid-solutions relevant for advanced nuclear fuels," Waste Management 2008 Conference Proceedings, Phoenix, AZ (March 2008). 


\subsection{Presentations and conference abstracts}

Shuller-Nickles, L., "Quantum-mechanical calculations and Monte Carlo simulations of the thermodynamic mixing properties for tetravalent AO2 (A = Zr, Ce, Th, U)," invited talk for Georgia Institute of Technology, Atlanta, GA (October 2011).

Shuller-Nickles, L., "Quantum-mechanical calculations and Monte Carlo simulations of the thermodynamic mixing properties for tetravalent AO2 (A = Zr, Ce, Th, U)," invited talk for University of South Carolina, Columbia, SC (October 2011).

Shuller, L.C., "Calculating the solid-state chemistry of nuclear materials: From nuclear fuel development to nuclear waste management," invited talk for National Institute of Standards and Technology, Gaithersburg, MD (February 2010).

Shuller, L.C., Ewing, R.C., and Becker, U., "Computational investigation of the thermodynamic mixing properties of actinide dioxide solid solutions," Materials Science and Technology, Pittsburgh, PA (October 2009).

Jianwei Wang, Rodney C. Ewing, and Udo Becker. Activation Energetics of Actinide Diffusion in $\mathrm{UO}_{2}$ from First-principles Calculations. Symposium Y: Actinides-Basic Science, Applications, and Technology. 2012 MRS Spring Meeting and Exhibit. April 9-13, 2012. San Francisco, California.

Jianwei Wang, Rodney C. Ewing, and Udo Becker. Effect of pressure on electronic structure and phase stability of hyperstoichiometric uranium dioxide. Materials Modeling and Simulation for Nuclear Fuels (MMSNF-2011) Aix en Provence, France September 26 to 28, 2011.

Bengtson A. K.; Ewing R. C.; Becker U. (2010) Ab initio calculations of He diffusion in apatite Conference on Goldschmidt 2010 - Earth, Energy, and the Environment Location: Knoxville, TN Date: JUN 13-18, 2010. Geochimica et Cosmochimica Acta, 74/12. Supplement: 1 Pages: A77-A77.

J.W. Wang, R.C. Ewing, and U. Becker. Amorphization and Disordering of Materials by Swift Heavy Ion: Atomic-Scale Heterogeneity Revealed by Combining the Thermal Spike Model and Molecular Dynamics Simulations. 2011 MRS Spring Meeting and Exhibit. April 25 - 29, 2011. San Francisco, California.

J.W. Wang, R.C. Ewing, and U. Becker. Effect of pressure on electronic structure and phase stability of hyperstoichiometric uranium dioxide. 241st ACS National Meeting \& Exposition, March 27-31, 2011, Anaheim, CA.

J.W. Wang, R.C. Ewing, and U. Becker. Electronic Structure and the Energetics of Actinide Diffusion in $\mathrm{UO}_{2}$. Plutonium Futures-Science-2010. Keystone, CO Keystone Resort \& Conference Center, September 19-23, 2010.

J. Wang and U Becker. First Principles Calculations of Diffusion Properties of Actinide Oxide Fuel Materials. Z: Actinides V - Basic Science, Applications, and Technology SESSION Z5: Chemistry of Nuclear Fuels. The 2010 Materials Research Society Spring. San Francisco, California, April 4-9, 2010. 


\title{
Thermodynamic properties of $\mathrm{Th}_{x} \mathrm{U}_{1-x} \mathrm{O}_{2}(0<x<1)$ based on quantum-mechanical calculations and Monte-Carlo simulations
}

\author{
Lindsay C. Shuller ${ }^{\mathrm{a}, \mathrm{b}, *}$, Rodney C. Ewing ${ }^{\mathrm{a}, \mathrm{b}, \mathrm{c}}$, Udo Becker $^{\mathrm{b}}$ \\ ${ }^{a}$ Materials Science Er Engineering, University of Michigan, Ann Arbor, MI 48109, USA \\ ${ }^{\mathrm{b}}$ Geological Sciences, University of Michigan, Ann Arbor, MI 48109, USA \\ ${ }^{c}$ Nuclear Engineering Er Radiological Sciences, Ann Arbor, MI 48109, USA
}

\section{A R T I C L E I N F O}

\section{Article history:}

Received 30 July 2010

Accepted 6 January 2011

Available online $\mathrm{xxxx}$

\begin{abstract}
A B S T R A C T
$\mathrm{Th}_{x} \mathrm{U}_{1-x} \mathrm{O}_{2+y}$ binary compositions occur in nature, uranothorianite, and as a mixed oxide nuclear fuel. As a nuclear fuel, important properties, such as the melting point, thermal conductivity, and the thermal expansion coefficient change as a function of composition. Additionally, for direct disposal of $\mathrm{Th}_{x} \mathrm{U}_{1-x} \mathrm{O}_{2}$, the chemical durability changes as a function of composition, with the dissolution rate decreasing with increasing thoria content. $\mathrm{UO}_{2}$ and $\mathrm{ThO}_{2}$ have the same isometric structure, and the ionic radii of 8-fold coordinated $\mathrm{U}^{4+}$ and $\mathrm{Th}^{4+}$ are similar (1.14 nm and $1.19 \mathrm{~nm}$, respectively). Thus, this binary is expected to form a complete solid solution. However, atomic-scale measurements or simulations of cation ordering and the associated thermodynamic properties of the $\mathrm{Th}_{x} \mathrm{U}_{1-x} \mathrm{O}_{2}$ system have yet to be determined. A combination of density-functional theory, Monte-Carlo methods, and thermodynamic integration are used to calculate thermodynamic properties of the $\mathrm{Th}_{x} \mathrm{U}_{1-x} \mathrm{O}_{2}$ binary $\left(\Delta H_{m i x}, \Delta G_{m i x}, \Delta S_{\text {mix }}\right.$, phase diagram). The Gibbs free energy of mixing $\left(\Delta G_{\text {mix }}\right)$ shows a miscibility gap at equilibration temperatures below $1000 \mathrm{~K}$ (e.g., $E_{\text {exsoln }}=0.13 \mathrm{~kJ} /(\mathrm{mol}$ cations $)$ at $\left.750 \mathrm{~K}\right)$. Such a miscibility gap may indicate possible exsolution (i.e., phase separation upon cooling). A unique approach to evaluate the likelihood and kinetics of forming interfaces between U-rich and Th-rich has been chosen that compares the energy gain of forming separate phases with estimated energy losses of forming necessary interfaces. The result of such an approach is that the thermodynamic gain of phase separation does not overcome the increase in interface energy between exsolution lamellae for thin exsolution lamellae $(10 \AA)$. Lamella formation becomes energetically favorable with a reduction of the interface area and, thus, an increase in lamella thickness to $>45 \AA$ A. However, this increase in lamellae thickness may be diffusion limited. Monte-Carlo simulations converge to an exsolved structure [lamellae || (21) 1 )] only for very low equilibration temperatures (below room temperature). In addition to the weak tendency to exsolve, there is an ordered arrangement of Th and $U$ in the solid solution [alternating $U$ and Th layers $\|\left\{\begin{array}{lll}1 & 0 & 0\end{array}\right\}$ ] that is energetically favored for the homogeneously mixed $50 \%$ Th configurations. Still, this tendency to order is so weak that ordering is seldom reached due to kinetic hindrances. The configurational entropy of mixing $\left(\Delta S_{\text {mix }}\right)$ is approximately equal to the point entropy at all temperatures, indicating that the system is not ordered.
\end{abstract}

(c) 2011 Elsevier B.V. All rights reserved.

\section{Introduction}

Thorium and uranium are the two heaviest naturally occurring elements, and both are utilized in nuclear fuels. While $0.7 \%$ of naturally occurring uranium is the fissile ${ }^{235} \mathrm{U}$ isotope, thorium only occurs as the fertile ${ }^{232} \mathrm{Th}$ isotope. However, ${ }^{232} \mathrm{Th}$ can be converted to ${ }^{233} \mathrm{U}$, which can be bred in both thermal and fast neutron reactors, because the value of $\eta$ (i.e., the mean number of fission neutrons produced per thermal neutron) is greater than 2.0 over a wide range of the thermal neutron spectrum [1]. The ${ }^{232} \mathrm{Th}$ to

* Corresponding author at: Geological Sciences, University of Michigan, Ann Arbor, MI 48109, USA.

E-mail address: Ishuller@umich.edu (L.C. Shuller).
${ }^{233} \mathrm{U}$ conversion occurs via the neutron absorption and subsequent $\beta$-decays in Reaction (1). Either fissile ${ }^{235} \mathrm{U}$ or ${ }^{239} \mathrm{Pu}$ is needed in Thfuels to initiate the fission required to convert fertile ${ }^{232} \mathrm{Th}$ to fissile ${ }^{233} \mathrm{U}$.

${ }^{232} \mathrm{Th} \underset{7.37 \text { barns }}{\stackrel{n, \gamma}{\rightarrow}}{ }^{233} \mathrm{Th} \underset{\tau_{1 / 2}=22}{\stackrel{\beta^{-}}{\rightarrow}}{ }_{\text {mins }}^{233} \mathrm{~Pa} \underset{\tau_{1 / 2}=27 \text { days }}{\stackrel{\beta^{-}}{\rightarrow}}{ }^{233} \mathrm{U}$

There has been renewed interest in Th-based fuels because of advantages related to proliferation resistance [1-3] and enhanced in-reactor performance [4-8]. Additionally, Th-based fuels have a higher chemical durability, as thorium only exists in its 4+ oxidation state, whereas uranium exists primarily in the $4+$ and $6+$ oxidation states. Due to the presence of two unpaired electrons for $\mathrm{U}^{4+}, \mathrm{UO}_{2}$ is easily oxidized in the presence of water and oxygen 
to a wide variety of $\mathrm{U}(\mathrm{VI})$-compounds [9]. The aqueous dissolution rate of $\mathrm{UO}_{2}$ is significantly decreased when doped with Th [10-14]. Thus, the addition of $\mathrm{ThO}_{2}$ improves the chemical durability of oxide fuels in a geologic repository.

Naturally occurring isometric thorianite, $\mathrm{ThO}_{2}$, is isostructural with uraninite, ideally $\mathrm{UO}_{2+x}$. Unit cell parameters change almost linearly according to Vegard's law with changing composition, which has lead most to conclude that this binary forms a complete solid solution $[15,16]$. However, mixed Th-U oxides, uranothorianite, may be exsolved at the nanoscale $[17,18]$. In addition, physico-chemical properties, such as sintering capability, depend on cation homogeneity, which can be controlled during synthesis $[19,20]$. Solid-state exsolution is the phase separation promoted by the thermodynamic properties of the solid solution. The same type of nano-scale exsolution and/or cation ordering of mixedTh-U nuclear fuels may affect important properties, such as thermal conductivity, the thermal expansion coefficient, and the melting point of such a solid solution. Thus, in addition to knowledge of the thermophysical and thermodynamic properties of Th-based fuels, an understanding of cation ordering of mixed oxide fuels is essential to fully understanding the in-reactor behavior of the fuel. Quantum-mechanical calculations and Monte-Carlo simulations of the complete $\mathrm{Th}_{x} \mathrm{U}_{1-x} \mathrm{O}_{2}$ solid-solution binary have been used to calculate the enthalpy of mixing $\left(\Delta H_{\text {mix }}\right)$. An idealized approach was taken to evaluate stoichiometric dioxides as a first approximation to the more complex hyperstoichiometric $\mathrm{Th}_{x} \mathrm{U}_{1-x} \mathrm{O}_{2+y}$. Subsequent thermodynamic integration was applied to derive the Gibbs free energy $\left(\Delta G_{m i x}\right)$ and configurational entropy $\left(\Delta S_{\text {mix }}\right)$ of mixing. The Gibbs free energy of mixing $\left(\Delta G_{\text {mix }}\right)$ is used to generate a binary temperature-composition phase diagram for $\operatorname{Th}_{x} \mathrm{U}_{1-x} \mathrm{O}_{2}$, and atomic-scale cation ordering is evaluated.

\section{Methodology}

Three consecutive computational methods were used to determine the solid-solution thermodynamic properties of $\mathrm{Th}_{x} \mathrm{U}_{1-x} \mathrm{O}_{2}$ : (1) quantum-mechanical geometry optimizations, (2) Monte-Carlo simulations, and (3) thermodynamic integration. The quantummechanical calculations were conducted in order to determine the optimized geometry of the end-members (i.e., $\mathrm{ThO}_{2}, \mathrm{UO}_{2}$ ), as well as intermediate compositions (e.g., $\mathrm{Th}_{x} \mathrm{U}_{1-x} \mathrm{O}_{2}$, where $0<x<1)$. The excess enthalpy of mixing was calculated from the quantum-mechanical results. The final energies from the quantum-mechanical calculations are then used to calculate cationcation interaction parameters for all possible pairs of exchangeable cations. For instance, in the $\mathrm{Th}_{x} \mathrm{U}_{1-x} \mathrm{O}_{2}$ series, interaction parameters are calculated for Th-U, Th-Th, and U-U for first-, second-, third-, and fifth-nearest-neighbor interactions. The interaction parameters are then used in the Monte-Carlo simulation to calculate temperature-dependent thermodynamic properties of the solid-solution series. Using a Monte-Carlo approach allows one to evaluate millions of configurations for different compositions in much larger supercells than is possible using quantum-mechanical calculations.

\subsection{Quantum-mechanical calculations}

The program CASTEP (CAmbridge Serial Total Energy Package; $[21,22])$ was used for the ground state total energy calculations. CASTEP is a density-functional theory-based code that uses plane waves as the basis function and pseudo-potentials to approximate the behavior of the core electrons. Ultra-soft pseudo-potentials were used to approximate the role of the core and inner valence electrons [23]. Outer valence electrons ( $U 5 f^{3} 6 s^{2} 6 p^{6} 6 d^{1} 7 s^{2}$, Th $6 s^{2} 6 p^{6} 6 d^{2} 7 s^{2}$, and $\left.02 s^{2} 2 p^{4}\right)$ are treated explicitly in the
Hamiltonian of the Schrödinger equation. The spin-polarized generalized gradient approximation (GGA) with the PW-91 [24] functional was used to approximate the electron exchange and correlation energies. GGA has shown greater structural agreement for $\mathrm{UO}_{2}$ geometry optimizations than local density approximation (LDA) [25]. The kinetic energy cut-off for the planewaves was $500 \mathrm{eV}$ and the $k$-point spacing was $0.1 \AA^{-1}$, which results in 9 $k$-points for the $2 \times 1 \times 1$ unit cell without symmetry constraints (i.e., P1). The total energy convergence tolerance was $1 \times 10^{-5}$ eV/atom. Geometry optimizations were performed without symmetry constraints, and the starting unit cell parameters for the intermediate structures were weighted averages of the endmember unit cell parameters. A spin-polarized approach was used for unit cells containing $U$ to take into account the behavior of the two unpaired 5 f electrons in $\mathrm{U}^{++}$.

Both binary oxides have the cubic fluorite structure, and each unit cell contains four formula units $(Z=4)$. The cation occupies every other cube formed by the oxygen sublattice (Fig. 1 ), resulting in four possible exchangeable cation sites for a $1 \times 1 \times 1$ unit cell and eight exchangeable cation sites for a $2 \times 1 \times 1$ unit cell. The four exchangeable cations of a unit cell are equidistant from each other, forming a tetrahedral coordination polyhedron around the oxygen.

\subsection{Generating interaction parameters}

The total energy as calculated by CASTEP is the formation energy from the zero valent gaseous species. The enthalpy of mixing from the quantum-mechanical calculations is determined as the difference between the total energy with some concentration $x$ of Th in $\mathrm{UO}_{2}$ and a linear combination of the total energies of the end-members (Eq. (2)). The quantum-mechanical calculations are ground-state $(0 \mathrm{~K})$ calculations; therefore, the enthalpy of mixing is considered to be an energy of mixing $\left(\Delta E_{\text {mix }}\right)$. The energy of mixing of Th into $\mathrm{UO}_{2}$ can also be described by a Hamiltonian that takes into account the interactions between the cations, i.e., the Th-Th, U-U, and Th-U interactions (Eq. (3)).

$$
\begin{aligned}
& \Delta E_{\text {mix }}^{\mathrm{CASTEP}}=E\left(\mathrm{Th}_{x} \mathrm{U}_{1-x} \mathrm{O}_{2}\right)-\left[x E\left(\mathrm{ThO}_{2}\right)+(1-x) E\left(\mathrm{UO}_{2}\right)\right] \\
& \Delta E_{\text {mix }}^{f i t}=E_{0}+\sum_{i}\left(n_{\mathrm{Th}-\mathrm{U}}^{i} E_{\mathrm{Th}-\mathrm{U}}^{i}+n_{\mathrm{Th}-\mathrm{Th}}^{i} E_{\mathrm{Th}-\mathrm{Th}}^{i}+n_{\mathrm{U}-\mathrm{U}}^{i} E_{\mathrm{U}-\mathrm{U}}^{i}\right)
\end{aligned}
$$

In Eq. (3), $i$ is the type of interaction as identified by the distance of the cation interaction (i.e., first-, second-, third-nearest-neighbor interaction), $n^{i}$ is the number of interactions of a type $i$ in a given cell configuration (e.g., $n_{\mathrm{Th}-\mathrm{U}}^{1}$ would be the number of first nearest-neighbor Th-U interactions), and $E^{i}$ is the energy contribution associated with a given cation-cation pair. The asymmetry of the system and the overall shape of a randomly ordered sub-regular solid solution are approximated by a Margules function (Eq. (4)). Later calculations are concerned with excess properties, so the interaction energies from Eq. (3) $\left(E^{i}\right)$ are combined into an exchange parameter $J^{i}$, which describes the energy associated with the Th$U$ cation exchange (Eq. (5)). The interaction parameters $J_{\mathrm{Th}-\mathrm{U}}^{i}$ and the Margules parameters $W_{1}$ and $W_{2}$ are fit to the quantummechanically determined energy of mixing according to Eq. (6). The interaction parameters for the solid-solution series and Margules parameters are listed in Table 1.

$$
\begin{aligned}
& E_{0}=x(1-x)\left[W_{1} x+W_{2}(1-x)\right] \\
& J_{\mathrm{Th}-\mathrm{U}}^{i}=E_{\mathrm{Th}-\mathrm{U}}^{i}-1 / 2\left(E_{\mathrm{Th}-\mathrm{Th}}^{i}+E_{\mathrm{U}-\mathrm{U}}^{i}\right) \\
& E_{\text {excess }}=E_{0}+\sum n_{\mathrm{Th}-\mathrm{U}}^{i} J_{\mathrm{Th}-\mathrm{U}}^{i}
\end{aligned}
$$


(a)

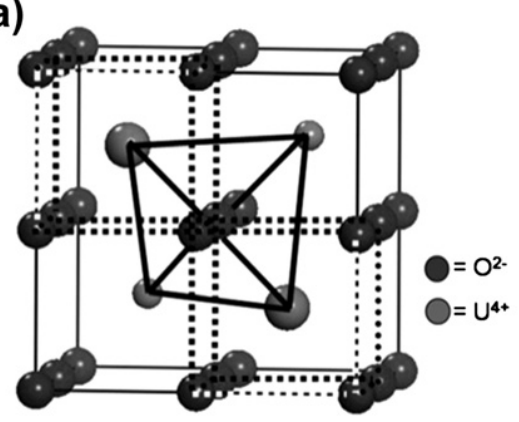

(b)

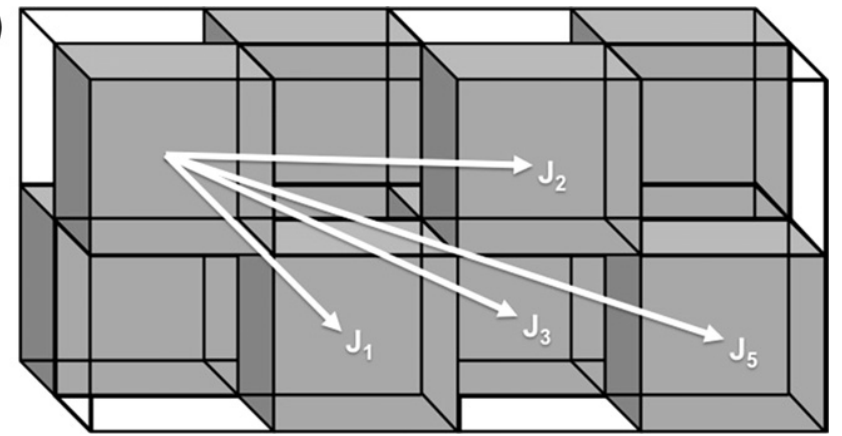

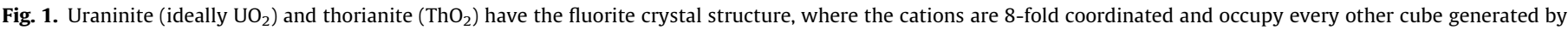

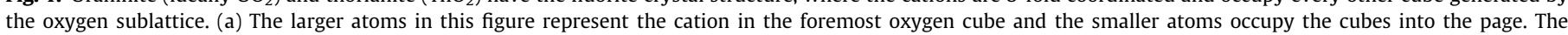

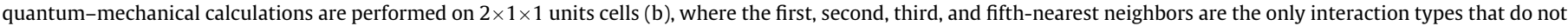
go beyond the unit cell. $J_{1}$ corresponds with a distance $r=3.86 \AA$, $J_{2}$ with $r=5.48 \AA$, $J_{3}$ with $r=6.69 \AA$, and $J_{5}$ with $r=8.65 \AA$.

Table 1

Margules and interaction parameters fit for $1 \times 1 \times 1$ unit cells and $2 \times 1 \times 1$ unit cells.

\begin{tabular}{|c|c|c|c|}
\hline Parameter & $\begin{array}{l}\text { Cation-cation } \\
\text { distance }(\AA)\end{array}$ & $1 \times 1 \times 1$ & $2 \times 1 \times 1$ \\
\hline $\begin{array}{l}W_{1}(\mathrm{~kJ} / \mathrm{mol} \text { exchangeable } \\
\text { cations })\end{array}$ & & 25.1 & 15.2 \\
\hline $\begin{array}{l}W_{2}(\mathrm{~kJ} / \mathrm{mol} \text { exchangeable } \\
\text { cations) }\end{array}$ & & 7.4 & 2.8 \\
\hline $\begin{array}{l}J_{1}(\mathrm{~J} / \mathrm{mol} \mathrm{Th}-\mathrm{U} \\
\quad \text { interactions })\end{array}$ & 3.86 & -3.0 & 24.0 \\
\hline $\begin{array}{l}J_{2}(\mathrm{~J} / \mathrm{mol} \mathrm{Th}-\mathrm{U} \\
\quad \text { interactions })\end{array}$ & 5.48 & $N / A$ & 24.0 \\
\hline $\begin{array}{l}J_{3}(\mathrm{~J} / \mathrm{mol} \mathrm{Th}-\mathrm{U} \\
\quad \text { interactions })\end{array}$ & 6.69 & $N / A$ & -2.0 \\
\hline $\begin{array}{l}J_{4}(\mathrm{~J} / \mathrm{mol} \mathrm{Th}-\mathrm{U} \\
\quad \text { interactions })\end{array}$ & 7.72 & $N / A$ & $\begin{array}{l}\text { Linearly } \\
\text { dependent } \rightarrow N / A\end{array}$ \\
\hline $\begin{array}{l}J_{5}(\mathrm{~J} / \mathrm{mol} \mathrm{Th}-\mathrm{U} \\
\quad \text { interactions })\end{array}$ & 8.65 & $N / A$ & -18.0 \\
\hline Correlation coefficient & & 0.926 & 0.960 \\
\hline
\end{tabular}

The quality of fit for the interaction parameters is evaluated by comparing the fit using different numbers of configurations and compositions across the $\mathrm{Th}_{x} \mathrm{U}_{1-x} \mathrm{O}_{2}$ binary. Two fit scenarios are compared in Table 1 . For a $1 \times 1 \times 1$ unit cell, the only type of cation-cation interaction, as identified by the distance between two cations, is the first nearest-neighbor interaction because all cations are at an equal distance $r$ from each other $(r=3.86 \AA)$. Due to the computational expense of the quantum-mechanical calculations, the expansion of the unit cell was limited to $2 \times 1 \times 1$. This unit cell expansion improved the fit for the exchange parameters, and increasing the number of configurations adds more energetic information on longer-range interactions. The types of cation-cation interactions increased from only first nearest-neighbor interactions to the inclusion of fifth nearestneighbor interactions. $J_{1-3}$ represent the first-, second-, and third-nearest neighbor interactions $\left(r_{1}=3.86 \AA, r_{2}=5.48 \AA\right.$, $\left.r_{3}=6.69 \AA\right)$, and $J_{5}$ represents the fifth-nearest neighbor interactions $\left(r_{5}=8.65 \AA\right)$. The forth nearest-neighbor interaction does not improve the fit because this parameter is linearly dependent on the first nearest-neighbor interaction. The absolute value of the first and fifth nearest-neighbor interaction parameters are about equal but opposite in sign. That is, if the system tends to have more nearest-neighbor U-Th interactions, homocationic $\mathrm{U}-\mathrm{U}$ and Th-Th fifth nearest-neighbor interactions would be favored and vice versa. The $2 \times 1 \times 1$ fit was used for the Monte-Carlo simulations to include the most information about the energetics of the Th-U system.

\subsection{Monte-Carlo simulations}

The exchange parameters, $J_{i}$, are used in the Monte-Carlo simulations to calculate the lattice energy for millions of different cation configurations. The excess enthalpy of mixing is calculated for the solid-solution series using the methodology previously described [26-28]. An energy $E$ is associated with each starting configuration, and the energy change associated with swapping cation positions is $\Delta E$. If $\Delta E$ is negative, meaning that the new configuration is more energetically favored than the previous configuration, the new configuration is accepted. If $\Delta E$ is positive, then the new configuration is accepted with a probability following a Boltzmann distribution. In practice, the swap is accepted if $\exp (-\Delta E / k T)$ is greater than a random number between 0 and 1 . An $8 \times 8 \times 8$ supercell of the conventional unit cell was constructed for the Monte-Carlo simulations, resulting in 2048 exchangeable cation sites. The entropy of mixing and Gibbs free energy of mixing are then calculated using the Bogoliubov integration scheme [28-30].

\section{Structure analysis of quantum-mechanical calculations}

The unit cell parameter and $\langle\mathrm{Me}-\mathrm{O}\rangle(\mathrm{Me}=\mathrm{U}$, Th $)$ bond lengths are compared to extended X-ray adsorption fine structure (EXAFS) and X-ray diffraction (XRD) measurements in order to validate the quantum-mechanical calculations. The relationship between the $\mathrm{Th}_{x} \mathrm{U}_{1-x} \mathrm{O}_{2}$ unit cell parameter and concentration of $\mathrm{Th}$, where $0<x<1$, follows Vegard's law (Fig. 2a); that is the deviation of the unit cell parameters from a line connecting uraninite and thorianite is less than the scatter of the experimental and the calculated values for different configurations. The calculated unit cell parameter for the end-members (Table 2 ) are within $\pm 0.3 \%$ of the experimental values, where the $\mathrm{UO}_{2}$ end-member is calculated to be $0.3 \%$ larger, and the $\mathrm{ThO}_{2}$ end-member is calculated to be $0.3 \%$ smaller than the experimental unit cell measurements [8,31-38]. The shorter $\langle$ Th- $\mathrm{O}\rangle$ distances are expected according to the findings that DFT-GGA calculations typically result in smaller unit cell parameters and shorter bond lengths. The longer $\langle\mathrm{U}-\mathrm{O}\rangle$ distances may be attributed to the commonly reported errors of DFT calculations on highly correlated $5 f$ electrons ( $\mathrm{U}^{4+}$ contains two). However, the reported lattice parameters for $\mathrm{UO}_{2}$ range from 5.468 to $5.471 \AA$ [ [31-38] (see Fig. 2a).

The $\langle\mathrm{Me}-\mathrm{O}\rangle$ bond lengths for the first-shell distances and $\langle\mathrm{Me}-$ $\mathrm{Me}\rangle$ bond lengths for the second-shell distances are compared to experimental EXAFS results, which show that the first-shell $\langle\mathrm{Me}-$ O) distances vary slightly across the solid solution range; however, second-shell $\langle\mathrm{Me}-\mathrm{Me}\rangle$ distances vary significantly with composition [31]. Similar results are observed for the calculated $\langle\mathrm{Me}-\mathrm{O}\rangle$ and $\langle\mathrm{Me}-\mathrm{Me}\rangle$ distances (Table 3). After correcting the bond lengths 

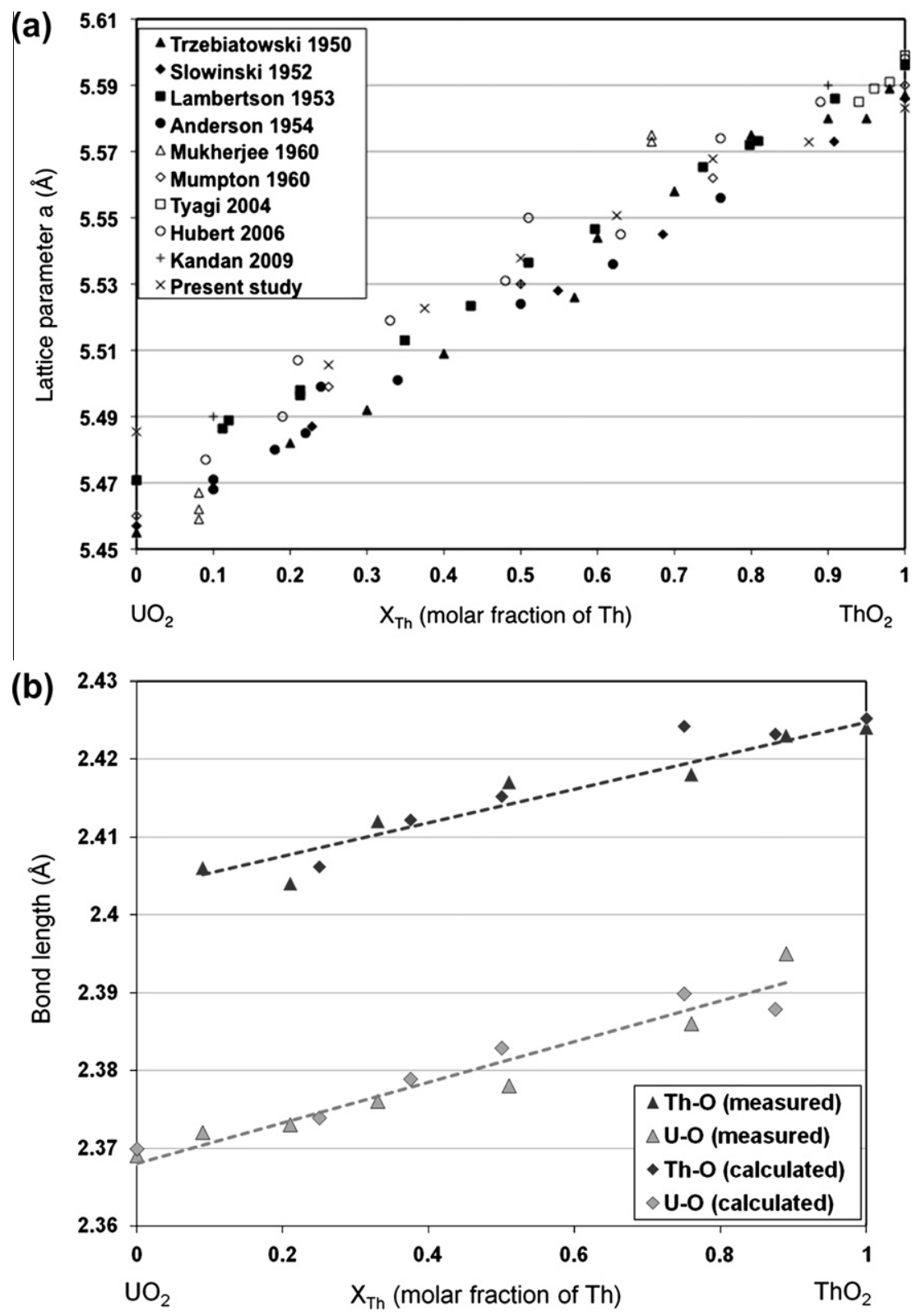

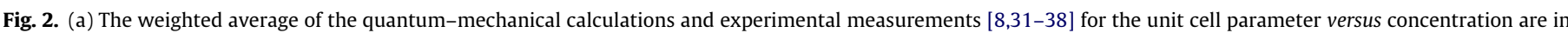

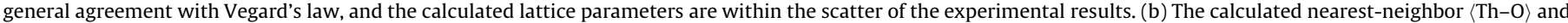
$\langle\mathrm{U}-\mathrm{O}\rangle$ bond lengths are corrected for the $0.3 \%$ mismatch in the unit cell parameter and are compared with bond lengths determined by EXAFS measurements [31].

Table 2

Comparison of calculated unit cell parameter with measured values [31].

\begin{tabular}{llll}
\hline Structure & Calculated $(\AA)$ & Measured $(\AA)$ & Difference $(\%)$ \\
\hline $\mathrm{ThO}_{2}$ & 5.583 & 5.598 & -0.3 \\
$\mathrm{UO}_{2}$ & 5.487 & 5.471 & 0.3 \\
\hline
\end{tabular}

based on the $0.3 \%$ mismatch in the unit cell parameter, the bond length as a function of Th concentration has no specific trend in excess bond length based on a linear combination of the endmembers (Fig. 2b).

\section{Thermodynamic properties of mixing}

The enthalpy of mixing is positive at all temperatures, indicating that this is not an ideal solid-solution, which is a first indication that some unmixing may occur (Fig. 3a). The absolute values of the maxima of the enthalpy of mixing, however, are relatively small, only about $3 \mathrm{~kJ} /(\mathrm{mol}$ exchangeable cations) at $1000 \mathrm{~K}$. For comparison, the maximum enthalpy of mixing at $1000 \mathrm{~K}$ for the $\mathrm{ThSiO}_{4}-$ $\mathrm{USiO}_{4}$ solid solution is about $11 \mathrm{~kJ} /(\mathrm{mol}$ exchangeable cations $)$ [28]. The enthalpy of mixing curves are asymmetric with a maximum around $x=0.62$ Th mole fraction; thus, the greatest amount of unmixing is expected to occur in this composition range. Lower enthalpy structures (i.e., an ordered structure and an exsolved structure) are marked in Fig. 3a and discussed below.

In order to quantify the degree of unmixing, the Gibbs free energy of mixing has to be evaluated. At temperatures below $1000 \mathrm{~K}$, the Gibbs free energy of mixing curves for the $\mathrm{Th}_{x} \mathrm{U}_{1-x} \mathrm{O}_{2}$ binary have a minimum at low and high Th concentrations, defining a miscibility gap (Fig. 3b). The contact points, where the tangent line touches the Gibbs free energy of mixing curve, define the minimum and maximum Th concentration between which exsolution 

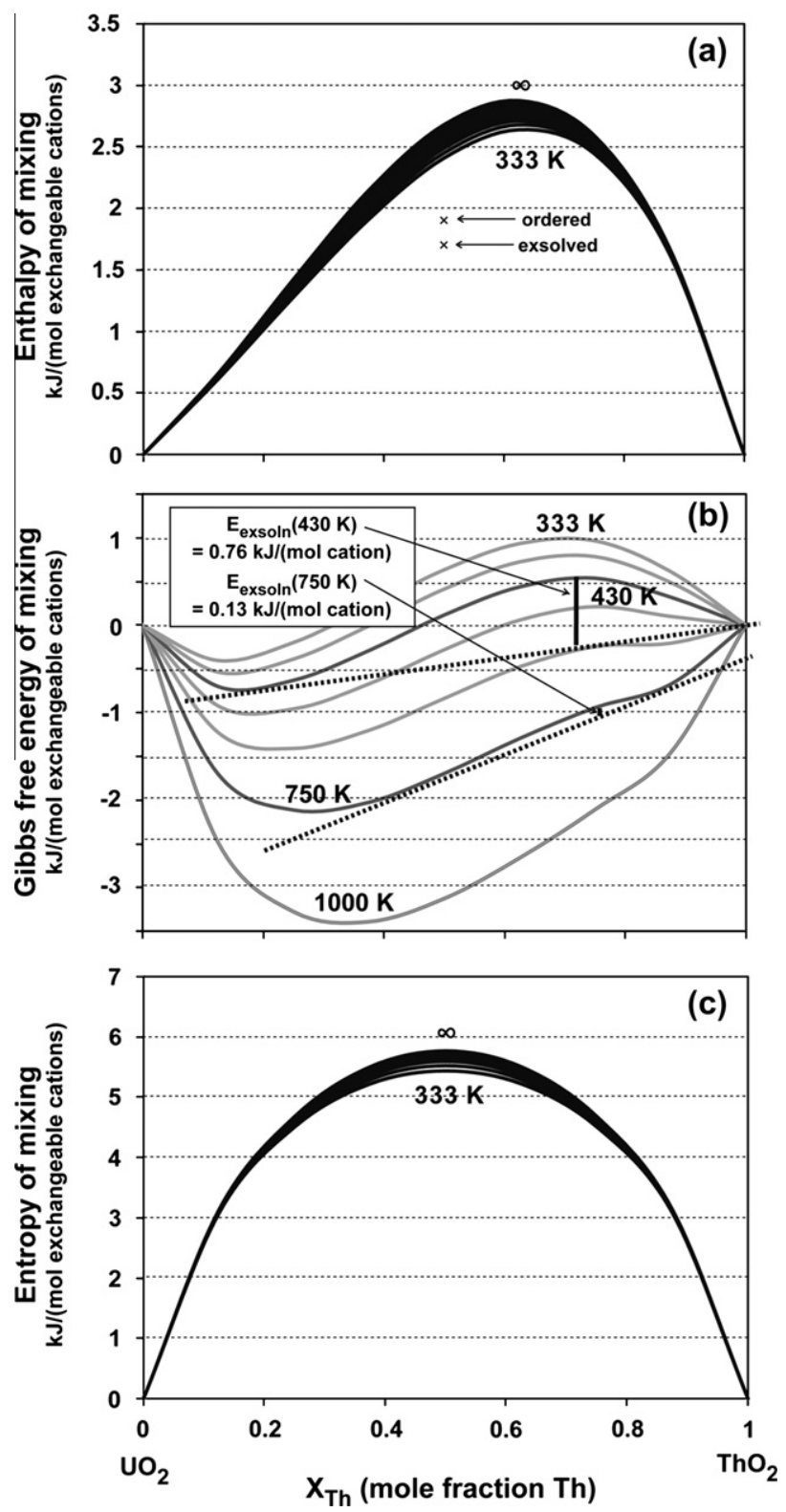

Fig. 3. Thermodynamic mixing properties with respect to the mole fraction of Th and temperature from the Monte-Carlo simulation. (a) Enthalpy of mixing with the enthalpy for the ordered and exsolved structures marked; (b) Gibbs free energy of mixing showing a miscibility gap below $750 \mathrm{~K}$, where the driving force $\left(E_{\text {exsoln }}\right)$ is labeled at $750 \mathrm{~K}$ and $430 \mathrm{~K}$; (c) Configurational entropy is nearly equal to the point entropy (configurational entropy without ordering) at all temperatures.

Table 3

Comparison of calculated $\langle\mathrm{Me}-\mathrm{O}\rangle(\mathrm{Me}=\mathrm{Th}, \mathrm{U})$ bond lengths with measured values [31]. The calculated values are averaged over all $\langle\mathrm{Me}-\mathrm{Me}\rangle$ and $\langle\mathrm{Me}-\mathrm{O}\rangle$ bonds within each configuration.

\begin{tabular}{llllllll}
\hline \multirow{2}{*}{ Solid } & \multicolumn{3}{l}{ Calculated distance $(\AA)$} & & \multicolumn{3}{l}{ Measured distance $(\AA)$} \\
\cline { 2 - 3 } & $\mathrm{Th}-\mathrm{O}$ & $\mathrm{U}-\mathrm{O}$ & $\langle\mathrm{Me}-\mathrm{Me}\rangle$ & & $\mathrm{Th}-\mathrm{O}$ & $\mathrm{U}-\mathrm{O}$ & $\langle\mathrm{Me}-\mathrm{Me}\rangle$ \\
\hline $\mathrm{ThO}_{2}$ & 2.42 & & 3.95 & & 2.42 & & 3.96 \\
$\mathrm{Th}_{0.5} \mathrm{U}_{0.5} \mathrm{O}_{2}$ & 2.41 & 2.39 & 3.91 & & 2.42 & 2.38 & 3.92 \\
$\mathrm{UO}_{2}$ & & 2.38 & 3.87 & & & 2.37 & 3.87 \\
\hline
\end{tabular}

can potentially occur. Thus, the solvus can be derived based on the intersection of the tangents to each temperature-dependent Gibbs free energy curve, and the resulting temperature-composition phase diagram for the $\mathrm{Th}_{x} \mathrm{U}_{1-x} \mathrm{O}_{2}$ binary is shown in Fig. 4. The phase diagram in Fig. 4 is, however, based on the assumption that all energy contributions are considered in the Monte-Carlo calculations and the subsequent thermodynamic integration. The validity of this assumption and additional energy contributions are discussed below. As shown in Fig. 3c, the configurational entropy at all temperatures is almost equal to the point entropy, which is the configurational entropy without ordering, indicating that the system is not converging to a specifically ordered state.

The lack of cation ordering, as indicated from the configurational entropy curves, is observed from the Monte-Carlo results at all calculated temperatures (Fig. 5a). However, based on the Gibbs free energy of mixing, exsolution is expected to occur below $1000 \mathrm{~K}$. Fig. 5b shows a completely mixed ordering scheme of alternating Th/U layers || to $\left(\begin{array}{lll}1 & 0 & 0\end{array}\right)$ that is homogeneous down to the scale of the original conventional unit cell. Symmetrically equivalent to this ordered structure are alternating Th/U layers II to $\left(\begin{array}{lll}0 & 0 & 1\end{array}\right)$ and $\left(\begin{array}{lll}0 & 1 & 0\end{array}\right)$. The $\left(\begin{array}{lll}1 & 0 & 0\end{array}\right)$ lamellar configuration has the lowest energy of the possible configurations based on an 8 cation unit cell with $50 \%$ Th concentration, where the energy is calculated using the interaction parameters for the configuration without optimization. Thus, this configuration is not a direct result of the interaction parameters. For example, the positive $J_{1}$ parameter indicates homocationic interactions are favored, and thus, does not support alternating cation layers in any direction. The interaction parameters $\left(J_{1}\right.$ through $\left.J_{5}\right)$ directly support the observed nanoexsolved structure with lamellae parallel to (21) $)$ (Fig. 5c), which is slightly more energetically favorable, according to the MonteCarlo simulations. The driving force, or respective energy to go from the mixed to the exsolved structure, is defined as the exsolution energy $\left(E_{\text {exsoln }}\right)$ labeled in Fig. $3 \mathrm{~b}$. A further complication arises from the fact that the exsolved layers, which are indexed to be $(21 \overline{1})$ in Fig. 5c, may be a linear combination of exsolution in the $\left\{\begin{array}{lll}1 & 1 & 1\end{array}\right\},\left\{\begin{array}{lll}1 & 0 & 0\end{array}\right\}$, and their symmetry-equivalent directions. That means that the exsolution pattern can change direction, similar to the changing direction of the oscillatory zoning observed in the experimental BSE image of grossular-andradite garnet by Pollok et al. [39]. Extremely low equilibration temperatures (<200 K) would be necessary to stabilize a lamellar structure, which would result in a substantially lower enthalpy of mixing for 0.5 mole fraction of Th $[1.69 \mathrm{~kJ} /(\mathrm{mol}$ exchangeable cations $)]$. If the system were to order, the enthalpy of mixing would be $1.87 \mathrm{~kJ} /$ (mol exchangeable cations) at 0.5 mole fraction of Th. However, energetic competition between ordering in different directions and exsolution in different directions out-weighs the driving forces for ordering and/or exsolution.

The driving force for the exsolution, $E_{\text {exsoln }}$, is determined as the difference between the maximum of the Gibbs free energy of mixing curve and the tangent line that connects the minima of the same curve. The tendency towards exsolution is exemplified in Fig. 6, which shows Gibbs free energy of mixing curves for three simulated binaries at a given temperature, $T$. The Gibbs free energy of mixing curves have different maxima at 0.5 mole fraction of Th; however, the phase diagram for each of the simulated binaries would be identical, solely based on the concentrations corresponding to the intersection of a tangent line through the minima. The system with the largest $E_{\text {exsoln }}$ has the greatest tendency for exsolution. The $E_{\text {exsoln }}$ for the $\mathrm{Th}_{x} \mathrm{U}_{1-x} \mathrm{O}_{2}$ binary at temperatures below $1000 \mathrm{~K}$ is small - from $0.13 \mathrm{~kJ} /$ (mol cations) at $750 \mathrm{~K}$ up to $1.12 \mathrm{~kJ} /(\mathrm{mol}$ cations $)$ at $333 \mathrm{~K}$ - indicating that the tendency towards exsolution is low. The Monte-Carlo simulations inherently "flatten" the Gibbs free energy curves because the cations are able to swap positions with cations some distance away. Basically, the simulations allow for unphysical long-distance cation exchanges in order to reach thermodynamic equilibrium quickly, which essentially avoids kinetically-hindered configurations (e.g., consisting of very thin exsolution lamellae with a number of interfaces 


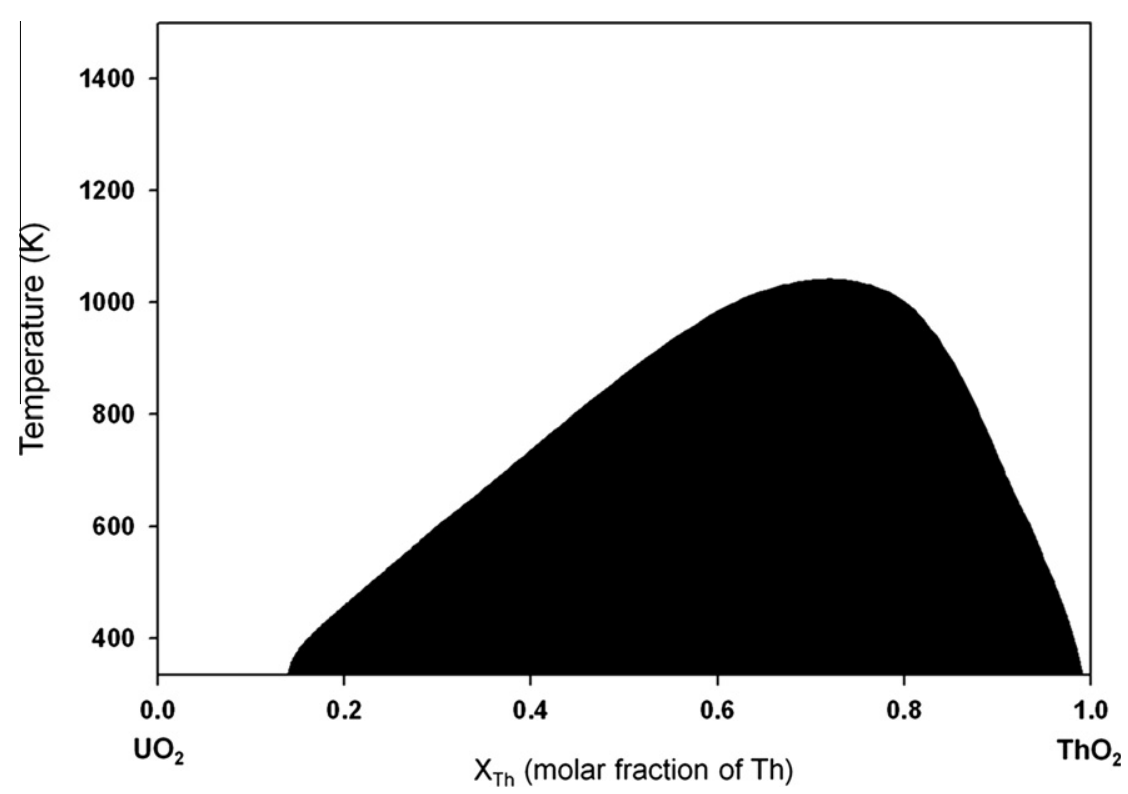

Fig. 4. Estimated temperature-composition phase diagram indicating the miscibility gap in the $\operatorname{Th}_{x} \mathrm{U}_{1-x} \mathrm{O}_{2}$ binary .

between U-rich and Th-rich phases) that would be caused by slow solid-state cation diffusion rates; however, diffusion is not explicitly considered during the Monte-Carlo simulation. Exsolution is further hindered by factors such as the buildup of a lattice mismatch at the interface. Low temperatures are necessary for exsolution (by producing high $E_{\text {exsoln }}$ values) to overcome the interfacial energy $\left(E_{I F}\right)$ of the exsolution lamellae; however, the same low temperatures would slow down the diffusion that is needed to actually generate low-energy exsolution features, such as lamellae.

Exsolution occurs when the $E_{\text {exsoln }}$ is greater than the $E_{I F}$, which is composed of a chemical and strain component. The chemical contributions (i.e., the local U-Th interactions across the interface) to the interface energy are contained in the interaction parameters, $J_{i}$. However, the strain that accumulates as a result of the lattice mismatch is not included because only small unit cells can be used in the quantum-mechanical calculations that are the basis for the derivation of the J's. A large quantum-mechanical unit cell would be required in order to calculate the strain between a U-rich and Th-rich phase, which would be computationally expensive. Thus, results from a study on the solid solution and phase separation of the isometric garnet compositions, andradite $\left[\mathrm{Ca}_{3} \mathrm{Fe}_{2}\left(\mathrm{SiO}_{4}\right)_{3}\right]$ and grossular $\left[\mathrm{Ca}_{3} \mathrm{Al}_{2}\left(\mathrm{SiO}_{4}\right)_{3}\right]$, are used as a rough estimate for the interface energy [40]. The garnet solid solution may be a suitable analogy because the grossular-andradite system has similar lattice mismatch $(1.8 \%)$ as the uraninite-thorianite system, and interfaces are chemically similar, i.e., between high-spin $\left(\mathrm{U}^{4+}\right.$ or $\mathrm{Fe}^{3+}$ ) and low-spin $\left(\mathrm{Th}^{4+}\right.$ or $\mathrm{Al}^{3+}$ ) phases. The $E_{I F}$ for the grossularandradite exsolution lamellae was calculated to be $\sim 1 \mathrm{meV} / \AA^{2}$ or $96.5 \times 10^{-3} \mathrm{~kJ} /\left(\mathrm{mol} \AA^{2}\right)$ [40], and this value is used in Eq. (7) to calculate the $E_{I F}$ for the $\mathrm{ThO}_{2}-\mathrm{UO}_{2}$ lamellae.

$$
\begin{aligned}
E_{I F}= & A\left(\AA^{2} / \text { unit cell }\right) \cdot E_{I F}^{\prime}\left(\mathrm{kJ} /\left(\mathrm{mol} \AA^{2}\right)\right) \\
& \cdot 1 / N_{c}(\text { unit cell } / \# \text { cations }) \\
= & 37778.72 \AA^{2} / \text { cell } \cdot 96.5 \times 10^{-3} \mathrm{~kJ} /\left(\mathrm{mol} \AA^{2}\right) \\
& \cdot 1 / 8198(\text { cell } / \# \text { cations }) \\
\approx & 0.4 \mathrm{~kJ} /(\text { mol cations })
\end{aligned}
$$

where $A$ is the area of the interface, $E_{I F}^{\prime}$ is the interface energy per unit area taken from the grossular-andradite example, and $N_{c}$ is the number of cations in the unit cell. The factor $1 / N_{c}$ is used to normalize the interface energy to the number of cations involved in the formation of the interfaces rather than an interface area, which makes it easier to compare $E_{I F}$ with $E_{\text {exsoln }}$. The total interface area was obtained by cleaving the $8 \times 8 \times 8$ supercell along the (21) $)$ starting at the first interface, resulting in a rectangular slab with 8 interchanging layers of $U$ and $T h$. The total interface area is $37,778 \AA^{2}$ /cell, and the total number of cations in the slab is 8198 cations/cell. The $E_{I F}$ for the exsolved $\mathrm{Th}_{0.5} \mathrm{U}_{0.5} \mathrm{O}_{2}$ structure is $0.4 \mathrm{~kJ} /(\mathrm{mol}$ exchangeable cations) - substantially larger than the $E_{\text {exsoln }}$ at $750 \mathrm{~K}$, which indicates that the driving force for exsolution cannot overcome the interface energy. The above estimate of the driving force to form lamellae versus the build-up of interface energy becomes more favorable for the former if the lamellae are thicker (i.e., less interface area per unit volume). In the above example, $E_{I F}$ and $E_{\text {exsoln }}$ become equal if the average thickness of the lamellae increases from $\sim 10 \AA$ (i.e., the lamellae are about 3 cation rows thick and spaced about $3.5 \AA$ apart) to $\sim 45 \AA$. However, slow diffusion requires much longer equilibration times. In addition, wider/thicker lamellae would have to be formed without going through the less energetically favored thinner ones, which is unlikely. These additional hindrances are even greater at lower temperatures. For example, the $E_{\text {exsoln }}$ at $333 \mathrm{~K}\left[E_{\text {exsoln }}=1.12 \mathrm{~kJ} /(\mathrm{mol}\right.$ exchangeable cations $)]$ is greater than the $E_{I F}[0.4 \mathrm{~kJ} /(\mathrm{mol}$ exchangeable cations)], yet exsolution is not observed in the Monte-Carlo simulation until below room temperature. The onset of exsolution is reached well before discernable exsolution lamellae are observed because the driving force for exsolution is small.

\section{Composition of natural and synthetic $\operatorname{Th}_{x} U_{1-x} O_{2}$}

One approach to validating the incorporation limit of $\mathrm{ThO}_{2}$ in $\mathrm{UO}_{2}$, as calculated in this study, is to compare the calculated results with analytical data on the compositions of natural and synthetic samples. The difficulty with such an approach is that most analytical methods do not distinguish between nano-scale exsolution features and truly homogeneous solid solutions. In addition, especially for synthetic samples, equilibrium is often not reached due to insufficient time for equilibration, unless samples are equilibrated at very high temperature for a long period of time, as in [19]. However, such a high temperature equilibration approach would not help in determining incorporation limits for low-temperature 
(a)

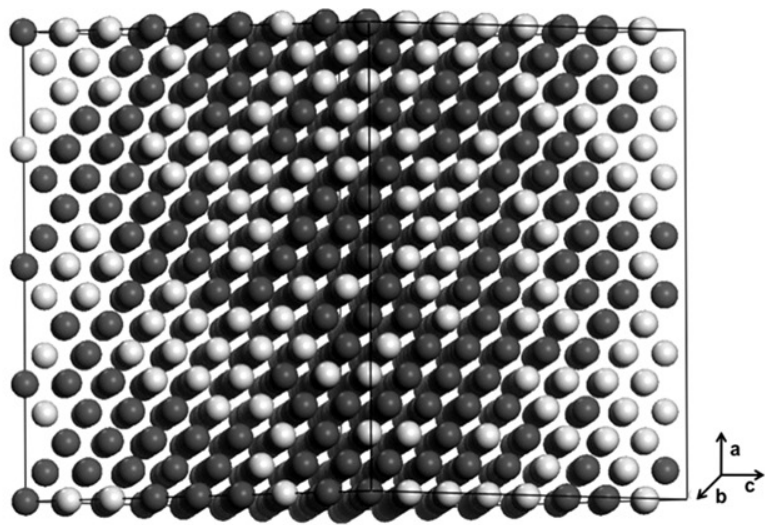

(b)

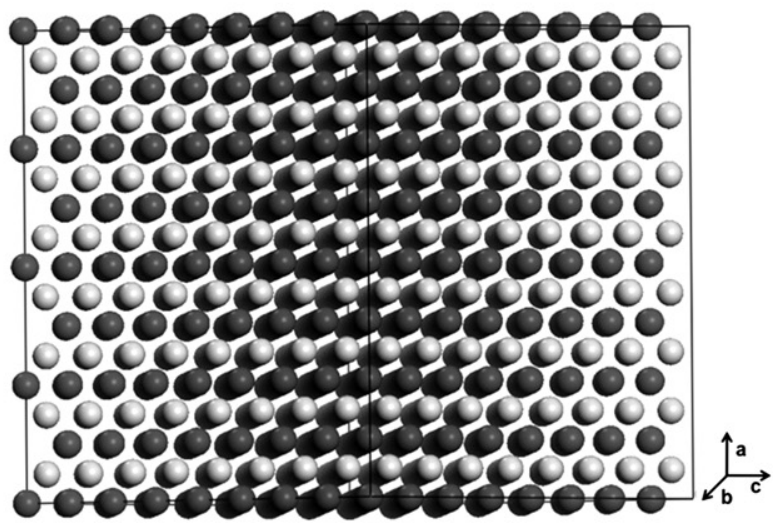

(c)

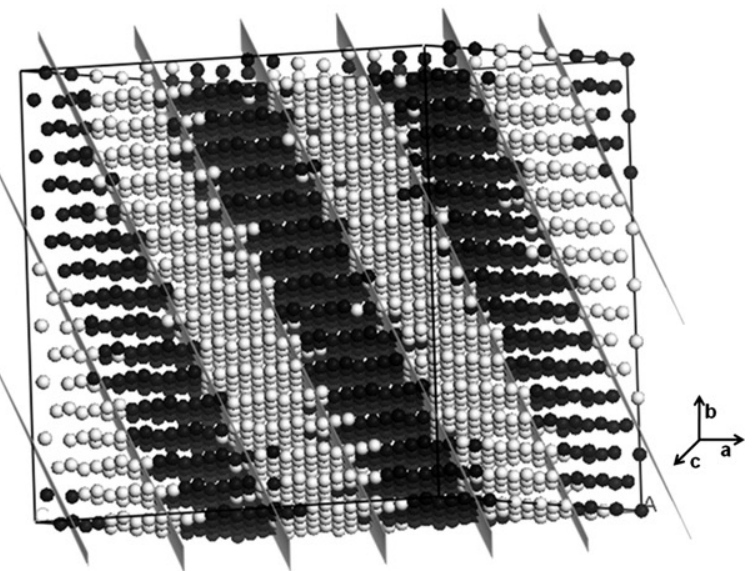

Fig. 5. Schematic of supercells indicating the (a) random arrangement of cations at temperatures above $200 \mathrm{~K}$, (b) alternating $U$ and Th layers $\|\left\{\begin{array}{lll}1 & 0 & 0\end{array}\right\}$, which is the most favorable unexsolved configuration for a 50:50 composition, and (c) exsolved lamellae structure $\|(21 \overline{1})$ at temperatures below $200 \mathrm{~K}$.

incorporation. Finally, whether the thermodynamically-possible incorporation limit is actually reached in natural samples may be limited by the composition of the fluid from which the solid solution formed.

Natural samples of uraninite and thorianite containing various amounts of Th and $U$, respectively, have been documented [16,41-44]. Uraninite samples from the natural fission reactor at Oklo-Okélobondo uranium deposit in southeast Gabon contain minimal amounts of $\mathrm{ThO}_{2}(<0.1 \mathrm{wt} . \%$ oxide) $[43,44]$. The OkloOkélobondo uranium deposits formed during the accumulation of oxidized U-rich saline fluids, which later came into contact with reducing oil-rich fluids, causing the reduction of $\mathrm{U}^{6+}$ to $\mathrm{U}^{4+}$. Thorium, which only occurs in the $4+$ oxidation state, would not have been mobilized in the oxidized U-rich saline fluid. Thus, Th is present as a fissiogenic daughter product of ${ }^{240} \mathrm{Pu}$ and ${ }^{236} \mathrm{U}$ generated by neutron-capture reactions [43]. Similarly, only minimal concentrations of $\mathrm{ThO}_{2}$ are found in sedimentary and vein-type uraninite deposits due the oxidation of uranium to $\mathrm{U}(\mathrm{VI})$ in solution prior to precipitation.

Of the three types of natural uraninite deposits (sedimentary, vein-type, or igneous), typically only the igneous deposits contain fractions of $\mathrm{ThO}_{2}$ greater than $1 \mathrm{~mol} \%[41,45,46]$. The composition of uraninite and thorianite from igneous rocks of the Grenville province of the Canadian Shield contain U:Th ratios ranging from 9.2 to 0.43 . The compositions of the uraninites were evaluated based on oxide weight\% of Th and slightly-oxidized U (7-55 wt.\% $\mathrm{ThO}_{2}$ and 25-73 wt.\% $\mathrm{U}_{3} \mathrm{O}_{8}$ ) [16]. Although the calculations are completed for the idealized stoichiometric solid solution, measurements from natural (non-stoichiometric) samples provide a general estimate for the compositional range. Frondel [42] noted that uraninites primarily contain $<20 \mathrm{wt} . \% \mathrm{ThO}_{2}$, and those containing equal amounts of $U$ and $T h$ are rare. Based on the compositions of natural samples, there is no clear evidence that the $\mathrm{Th}_{x} \mathrm{U}_{1-\chi} \mathrm{O}_{2}$ binary is a continuous solid solution.

Chemical analysis and XRD data for synthetic $\mathrm{Th}_{x} \mathrm{U}_{1-x} \mathrm{O}_{2}$ support the hypothesis of complete solid solution, based on the agreement of the unit cell parameters with Vegard's law, which is an indirect measure that indicates the linearity of the energetics of the binary [8,34-37]. The unit cell parameters of natural and synthetic samples are typically measured using bulk analysis techniques, such as XRD; thus, potential local expansion or contraction beyond Vegard's law would be difficult to detect. In addition, Vegard's law is only a rough indication of the thermodynamics across a solid-solution series. The synthesis (or subsequent calcination) temperatures for typical specimens are above $1273 \mathrm{~K}$ and can be as high as $1973 \mathrm{~K}$ (e.g., [19]), which is well above the critical temperature based on the calculations in this study. Thus, if the synthetic samples were cooled rapidly, a homogeneous composition would be quenched. The Monte-Carlo simulations indicate that too low of a temperature is necessary for exsolution to occur; thus, possible heterogeneities are more likely due to the kinetics of growth processes. For example, the oscillatory zoning in garnets is driven by the kinetics of growth processes, not the non-ideality of the solid solution because the miscibility gap forms at too low of a temperature [39]. The synthesis route for $\mathrm{Th}_{x} \mathrm{U}_{1-x} \mathrm{O}_{2}$ solid solutions greatly influences the degree of homogeneity, where wet chemistry methods result in higher homogeneity than dry chemistry methods $[19,20]$.

\section{Comparison with calorimetric measurements}

Calorimetric measurements can be used to determine the energetic state of intermediate compositions for direct comparison to the calculated thermochemical parameters of the solid solution. The calculated enthalpies and Gibbs free energies are reported as enthalpies of mixing and Gibbs free energies of mixing. Thermodynamic mixing properties are calculated in reference to a linear combination of the end-members and can be directly compared to measured thermodynamic mixing properties. Experimentally, mixing properties are determined from measuring enthalpies of formation or Gibbs free energy of formation of end-members and intermediate members. The excess properties of the intermediate compositions are then evaluated with respect to a molar fraction of the end-members. Calorimetric measurements can measure enthalpies of formation and, in turn, enthalpies of mixing (e.g., $[47,48])$; however, such measurements for intermediate compositions across the $\mathrm{ThO}_{2}-\mathrm{UO}_{2}$ solid solution are not yet available. 


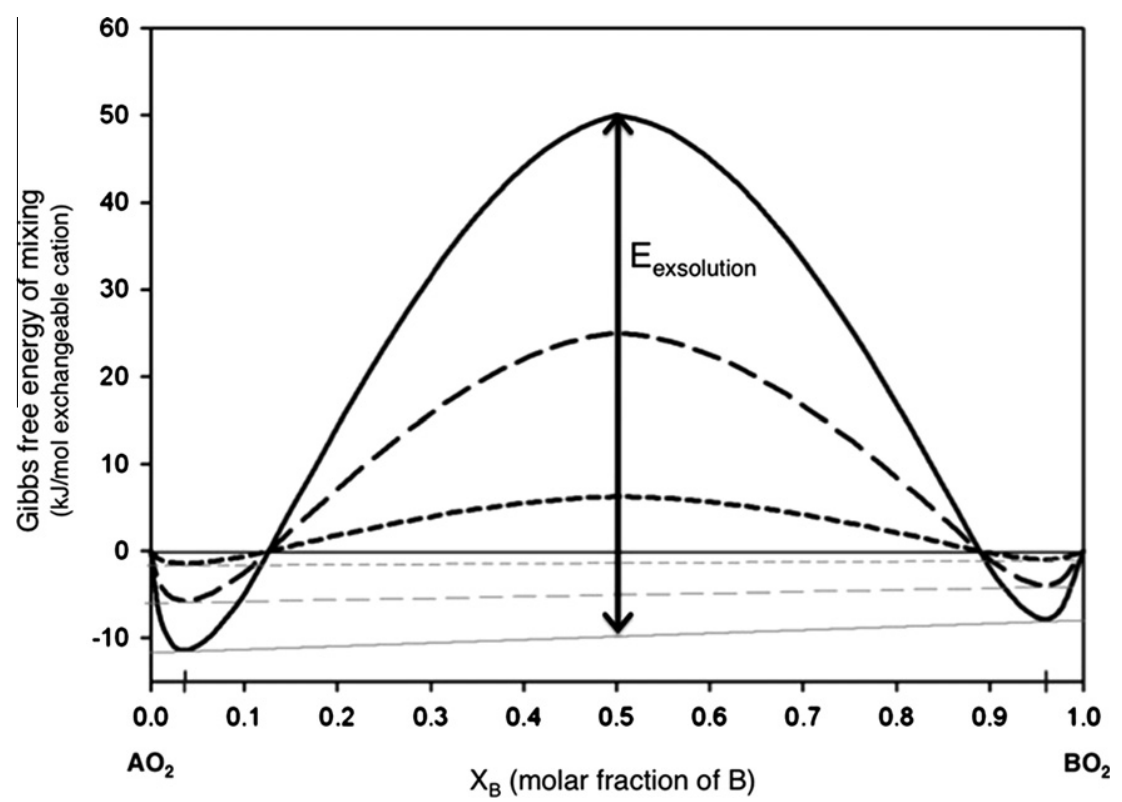

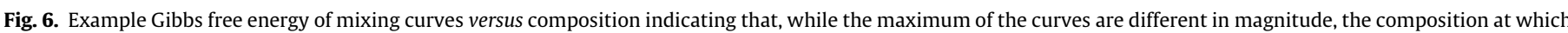
the tangential line to the minima intersects each curve is the same.

Alternatively, enthalpy increments and heat capacities are calorimetric measurements that are commonly available for intermediate compositions across the $\mathrm{ThO}_{2}-\mathrm{UO}_{2}$ solid solution $[38,49-$ 53]; however, enthalpy increments and heat capacities cannot be compared with the results of the calculations in this study. Dash et al. [53] compute Gibbs free energies of mixing from old vapor pressure data [54], which have since been disregarded [51]. The computed Gibbs free energy of mixing from Dash et al. [53] is less than $-15 \mathrm{~kJ} / \mathrm{mol}$ at $x=0.5$ and $T=1500 \mathrm{~K}$ for $\mathrm{Th}_{x} \mathrm{U}_{1-x} \mathrm{O}_{2}$. When compared with the maximum configurational entropy (i.e., $T \Delta S=-\mathrm{RT}(x \ln x+(1-x) \ln (1-x)$, using the point entropy where no ordering is considered), their $\Delta H$ data is roughly $-6.4 \mathrm{~kJ} / \mathrm{mol}$. However, due to the nearly ideal behavior as is evidenced by the unit cell parameters [31-38] and vapor pressures [51,54], the enthalpy of mixing should be low. Unfortunately, systematic measurements of $\Delta G_{\text {mix }}$ for the full solid solution from $\mathrm{ThO}_{2}$ to $\mathrm{UO}_{2}$ are currently not available; therefore, only the comparison with unit cell parameter measurements (Section 3) and the comparison with natural and synthetic Th concentrations (Section 5 ) have been used to validate the computational results.

\section{Implications for $\mathrm{Th}_{x} \mathrm{U}_{1-x} \mathrm{O}_{2}$ solid solutions}

The cation ordering determined from the Monte-Carlo simulations and the phase diagram generated from the calculated Gibbs free energy of mixing indicate that the $\mathrm{Th}_{x} \mathrm{U}_{1-x} \mathrm{O}_{2}$ system has a small tendency to exsolve below $1000 \mathrm{~K}\left(727^{\circ} \mathrm{C}\right)$; however, exsolution lamellae are observed only for calculations at temperatures below $200 \mathrm{~K}$. The only experimental evidence that may indicate exsolution in the $\mathrm{Th}_{x} \mathrm{U}_{1-x} \mathrm{O}_{2}$ binary is the sharp increase in magnetic susceptibility at $55 \mathrm{~mol} \% \mathrm{ThO}_{2}$ in $\mathrm{UO}_{2}$ [37]. However, nanoscale exsolution cannot be detected from bulk analysis techniques (e.g., XRD). Natural and synthetic samples could be homogeneous, and appear completely miscible, due to quenching from the high synthesis temperatures $\left(>1000^{\circ} \mathrm{C}\right)$. In addition, kinetic hindrances due to slow cation diffusion rates are expected to inhibit the formation of an interface between a Th-rich and U-rich phase or lamella.
While computational results indicate nano-scale formation of interfaces (shown in Fig. 5c), analysis of nano-scale features in experiments is often difficult because one has to have nano-scale resolution, and the nano-scale lamellae resulting from the formation of interfaces are often difficult to detect. For example, transmission electron microscopy has been used to resolve the nano-scale exsolution lamella in the hematite-ilmenite solid solution, which provides details about unusual magnetization features [18]. However, few studies exist in which such high-resolution techniques are used to re-evaluate previously studied solid solutions. Ordering within the unit cell, as shown in Fig. 5b, but extending only to the range of a nano-domain is often even harder to detect experimentally. To a certain degree, one would be able to detect the internal domain structure (e.g., number of Th-U interactions in the first coordination shell) using EXAFS. However, the local structure information obtained using EXAFS is spatially averaged. To the authors' knowledge, an atomic-scale investigation of the energetics and cation ordering in the $\mathrm{Th}_{x} \mathrm{U}_{1-x} \mathrm{O}_{2}$ system has not been conducted. However, according to the computational analysis exsolution temperatures are so low that exsolution in natural uranothorianite and synthetic $\mathrm{Th}_{x} \mathrm{U}_{1-x} \mathrm{O}_{2}$ is not expected.

\section{Acknowledgements}

The authors acknowledge support from the US Department of Energy, Nuclear Energy University Program grant (DE-AC0705ID14517). LCS was supported by an Office of Civilian and Radioactive Waste Management Graduate Fellowship from the USDOE. We especially thank Ron Fleming for inspiring our interest in the Th-fuel cycle and Th-based nuclear fuels. We are particularly grateful to Alex Navrotsky for her critical review of this study and very helpful insights into the interpretation of the experimental data.

\section{References}

[1] IAEA, Thorium fuel cycle - Potential benefits and challenges, TECDOC-1450, International Atomic Energy Agency, 2005.

[2] J.S. Herring, P.E. MacDonald, K.D. Weaver, C. Kullberg, Nucl. Eng. Des. 203 (2001) 65.

[3] P.E. MacDonald, C.B. Lee, Nucl. Technol. 147 (2004) 1.

[4] I.S. Kurina, L.S. Gudkov, V.N. Rumyantsev, At. Energy 92 (2002) 461. 
[5] T.R.G. Kutty et al., J. Nucl. Mater. 373 (2008) 309.

[6] M. Murabaya, S. Namba, Y. Takahash, T. Mukaibo, J. Nucl. Sci. Technol. - Tokyo 6 (1969) 128.

[7] Y. Takahashi, M. Murabayashi, J. Nucl. Sci. Technol. 12 (1975) 133.

[8] A.K. Tyagi, M.D. Mathews, B.R. Ambekar, R. Ramachandran, Thermochim. Acta 421 (2004) 69.

[9] F.N. Skomurski, L.C. Shuller, R.C. Ewing, U. Becker, J. Nucl. Mater. 375 (2008) 290.

[10] P.A. Demkowicz et al., Nucl. Technol. 147 (2004) 157.

[11] S. Hubert, K. Barthelet, B. Fourest, G. Lagarde, N. Dacheux, N. Baglan, J. Nucl. Mater. 297 (2001) 206.

[12] G. Heisbourg, S. Hubert, N. Dacheux, J. Ritt, J. Nucl. Mater. 321 (2003) 141

[13] G. Heisbourg, S. Hubert, N. Dacheux, J. Purans, J. Nucl. Mater. 335 (2004) 5.

[14] S. Hubert, G. Heisbourg, N. Dacheux, P. Moisy, Inorg. Chem. 47 (2008) 2064.

[15] R.M. Hazen, R.C. Ewing, D.A. Sverjensky, Am. Miner. 94 (2009) 1293.

[16] S.C. Robinson, A.P. Sabina, Am. Miner. 40 (1955) 624.

[17] U. Becker, P. Risthaus, F. Brandt, D. Bosbach, Chem. Geol. 225 (2006) 244.

[18] S.A. McEnroe, R.J. Harrison, P. Robinson, F. Langenhorst, Geophys. J. Int. 151 (2002) 890.

[19] N. Hingant, N. Clavier, N. Dacheux, N. Barre, S. Hubert, S. Obbade, F. Taborda, F. Abraham, J. Nucl. Mater. 385 (2009) 400.

[20] N. Hingant, N. Clavier, N. Dacheux, S. Hubert, N. Barre, R. Podor, L. Aranda, Powder Tech., (2010), doi:10.1016/j.powtec.2010.08.042.

[21] M.C. Payne, M.P. Teter, D.C. Allan, T.A. Arias, J.D. Joannopoulos, Rev. Mod. Phys. 64 (1992) 1045.

[22] M.D. Segall et al., J. Phys. Condens. Matter 14 (2002) 2717.

[23] D. Vanderbilt, Phys. Rev. B 41 (1990) 7892.

[24] J.P. Perdew, Y. Wang, Phys. Rev. B 45 (1992) 13244.

[25] F.N. Skomurski, R.C. Ewing, A.L. Rohl, J.D. Gale, U. Becker, Am. Miner. 91 (2006) 1761.

[26] M. Reich, U. Becker, Chem. Geol. 225 (2006) 278.

[27] E.D.A. Ferriss, E.J. Essene, U. Becker, Eur. J. Mineral. 20 (2008) 745

[28] E.D.A. Ferriss, R.C. Ewing, U. Becker, Am. Miner. 95 (2010) 229.

[29] E.R. Myers, Phys. Chem. Miner. 25 (1998) 465.
[30] J.M. Yeomans, Statistical Mechanics of Phase Transitions, Oxford Science Publications, Clarendon Press, Oxford, 1992

[31] S. Hubert, J. Purans, G. Heisbourg, P. Moisy, N. Dacheux, Inorg. Chem. 45 (2006) 3887.

[32] J.S. Anderson, D.N. Edgington, L.E.J. Roberts, E. Wait, J. Chem. Soc. (1954) 3324.

[33] B. Mukherjee, Nature 186 (1960) 464.

[34] F.A. Mumpton, R. Roy, J. Am. Ceram. Soc. 43 (1960) 234.

[35] W.A. Lambertson, M.H. Mueller, F.H. Gunzel, J. Am. Ceram. Soc. 36 (1953) 397.

[36] E. Slowinski, N. Elliott, Acta Crystallogr. 5 (1952) 768.

[37] W. Trzebiatowski, P.W. Selwood, J. Am. Chem. Soc. 72 (1950) 4504.

[38] R. Kandan, R. Babu, P. Manikandan, R.V. Krishnan, K. Nagarajan, J. Nucl. Mater. 384 (2009) 231.

[39] K. Pollok, B. Jamtveit, A. Putnis, Contrib. Mineral. Petrol. 141 (2001) 358

[40] U. Becker, K. Pollok, Phys. Chem. Miner. 29 (2002) 52

[41] R.M. Berman, Am. Miner. 42 (1957) 705.

[42] C. Frondel, Systematic mineralogy of uranium and thorium, Geological Survey Bulletin 1064 (US GPO, Washington, [DC]) 1958, pp. viii, 400 p.

[43] K.A. Jensen, R.C. Ewing, Geol. Soc. Am. Bull. 113 (2001) 32.

[44] L.Z. Evins, K.A. Jensen, R.C. Ewing, Geochim. Cosmochim. Acta 69 (2005) 1589.

[45] R.J. Finch, R.C. Ewing, J. Nucl. Mater. 190 (1992) 133.

[46] J. Janeczek, R.C. Ewing, J. Nucl. Mater. 190 (1992) 157.

[47] T.A. Lee, C.R. Stanek, K.J. McClellan, J.N. Mitchell, A. Navrotsky, J. Mater. Res. 23 (2008) 1105

[48] M. Aizenshtein, T.Y. Shvareva, A. Navrotsky, J. Am. Ceram. Soc. 93 (2010) 4142.

[49] J.K. Fink, Int. J. Thermophys. 3 (1982) 165.

[50] D.F. Fischer, J.K. Fink, L. Leibowitz, J. Belle, J. Nucl. Mater. 118 (1983) 342.

[51] K. Bakker, E.H.P. Cordfunke, R.J.M. Konings, R.P.C. Schram, J. Nucl. Mater. 250 (1997) 1.

[52] R. Agarwal, R. Prasad, V. Venugopal, J. Nucl. Mater. 322 (2003) 98.

[53] S. Dash, S.C. Parida, Z. Singh, B.K. Sen, V. Venugopal, J. Nucl. Mater. 393 (2009) 267.

[54] C.A. Alexander, J.S. Ogden, G.W. Cunningham, Battelle Memorial Institute Report BMI-1789, 1967. 


\title{
Atomistic calculations of the thermodynamic properties of mixing for tetravalent metal dioxide solid solutions: $(\mathrm{Zr}, \mathrm{Th}, \mathrm{Ce}) \mathrm{O}_{2}$
}

\author{
L.C. Shuller-Nickles ${ }^{\mathrm{a}, *}$, R.C. Ewing ${ }^{\mathrm{b}}$, U. Becker ${ }^{\mathrm{b}}$ \\ a Environmental Engineering and Earth Science, Clemson University, 342 Computer Court, Anderson, SC 29625, USA \\ ${ }^{\mathrm{b}}$ Earth and Environmental Sciences, University of Michigan, 1100 N. University Avenue, Ann Arbor, MI 48109, USA
}

\section{A R T I C L E I N F O}

\section{Article history:}

Received 22 May 2012

Received in revised form

10 August 2012

Accepted 13 August 2012

Available online 31 August 2012

Keywords:

Thermodynamic mixing properties

Cation ordering

Zirconia

Ceria

Thoria

Density functional theory

\begin{abstract}
A B S T R A C T
The thermodynamic mixing properties for isometric $\mathrm{Th}_{x} \mathrm{Ce}_{1-x} \mathrm{O}_{2}, \mathrm{Ce}_{x} \mathrm{Zr}_{1-x} \mathrm{O}_{2}$, and $\mathrm{Th}_{x} \mathrm{Zr}_{1-x} \mathrm{O}_{2}$ were determined using quantum-mechanical calculations and subsequent Monte-Carlo simulations. Although the $\mathrm{Th}_{x} \mathrm{Ce}_{1-x} \mathrm{O}_{2}$ binary indicates exsolution below $600 \mathrm{~K}$, the energy gain due to exsolution is small ( $E_{\text {exsoln }}=1.5 \mathrm{~kJ} /(\mathrm{mol}$ cations) at $200 \mathrm{~K})$. The energy gain for exsolution is significant for the binaries containing $\mathrm{Zr}$; at $1000 \mathrm{~K}, E_{\text {exsoln }}=6 \mathrm{~kJ} /\left(\mathrm{mol}\right.$ cations) for the $\mathrm{Ce}_{x} \mathrm{Zr}_{1-x} \mathrm{O}_{2}$ binary, and $E_{\text {exsoln }}=17 \mathrm{~kJ} /(\mathrm{mol}$ cations $)$ for the $\mathrm{Th}_{x} \mathrm{Zr}_{1-x} \mathrm{O}_{2}$ binary. The binaries containing $\mathrm{Zr}$ have limited miscibility and cation ordering (at $200 \mathrm{~K}$ for $x=0.5$ ). At $1673 \mathrm{~K}$, only 4.0 and $0.25 \mathrm{~mol} \% \mathrm{ZrO}_{2}$ can be incorporated into $\mathrm{CeO}_{2}$ and $\mathrm{ThO}_{2}$, respectively. Solid-solution calculations for the tetragonal $\mathrm{Th}_{x} \mathrm{Zr}_{1-x} \mathrm{O}_{2}$ binary show decreased mixing enthalpy due to the increased end-member stability of tetragonal $\mathrm{ZrO}_{2}$. Inclusion of the monoclinic $\mathrm{ZrO}_{2}$ is predicted to further reduce the mixing enthalpy for binaries containing $\mathrm{Zr}$.
\end{abstract}

(c) 2012 Elsevier Inc. All rights reserved.

\section{Introduction}

Several $\mathrm{AX}_{2}$ oxides are known to have the isometric fluorite, $\mathrm{CaF}_{2}$, structure, including those relevant to current and advanced nuclear fuels (e.g., $\mathrm{UO}_{2}, \mathrm{ThO}_{2}$ ). Mixed-Th-U fuels are under active investigation [1] and have been tested in power reactors (e.g., Shippingport and Fort St. Vrain in the United States and KAPS in India [2]). For such mixed Th-U fuels, $\mathrm{Zr}$ and Ce may be present as part of the initial fuel matrix (e.g., inert matrix fuel) or part of the final fuel matrix as fission products.

Ce is often used experimentally as a surrogate for Pu because of their similarities in atomic radii and valence states. In addition, the $\mathrm{Ce}-\mathrm{Zr}$ dioxide binary has been extensively studied for use as an automobile exhaust catalyst, fuel-cell electrolyte, and oxygen gas sensor [3-5]. At high temperatures, $\mathrm{ZrO}_{2}$, which has been proposed as an inert matrix fuel [6], has the isometric fluorite structure and undergoes a phase transformation with decreasing temperature, becoming tetragonal and finally, monoclinic baddeleyite at room temperature. The isometric actinide dioxides are thought to form complete solid solutions with each other due to their similar crystal structures and cation ionic radii. The homogeneity of these solid solutions, as well as the thermodynamic properties of mixing, is needed in order to fully understand their behavior during fuel

\footnotetext{
* Corresponding author.

E-mail addresses: 1shulle@clemson.edu (L.C. Shuller-Nickles) rodewing@umich.edu (R.C. Ewing), ubecker@umich.edu (U. Becker).
}

fabrication, in reactor, and finally, as a nuclear waste form. An extensive atomistic evaluation of the $\mathrm{Th}_{x} \mathrm{U}_{1-x} \mathrm{O}_{2}$ binary was previously reported [7]. This investigation includes other dioxide solid solutions, $\mathrm{ZrO}_{2}-\mathrm{CeO}_{2}-\mathrm{ThO}_{2}$, that contain tetravalent cations with unoccupied $f$ orbitals. The enthalpy of mixing and critical temperature at which the miscibility gap closes were recently described for the $\mathrm{Th}_{x} \mathrm{Ce}_{1-x} \mathrm{O}_{2}$ binary based on calorimetric measurements and atomistic simulations [8]. The thermodynamic mixing properties of these dioxide solid solutions are calculated, including the enthalpy of mixing, Gibbs free energy of mixing, and configurational entropy of mixing. From these properties, one can identify any tendency toward the formation of exsolution lamellae or stable ordered phases. The mixing in these binaries may affect physical and chemical properties, such as thermal conductivity, which will influence the performance of nuclear fuels in reactors and behavior of used nuclear fuels after disposal.

\section{Methods}

Quantum-mechanical (QM) geometry optimizations were used to calculate the enthalpy of mixing for a variety of compositions and cation configurations. QM calculations were used to capture electronic information, which is important for the evaluation of cation ordering. The geometry optimizations were performed using the planewave pseudopotential code CASTEP [9], where planewaves were used as functions for the overall wave function and the pseudopotentials mimic the role of the core electrons and 
their interactions with the valence electrons. The outer valence electrons $\left(Z r 4 s^{2} 4 p^{6} 4 d^{2} 5 s^{2}\right.$, Ce $4 f^{1} 5 s^{2} 5 p^{6} 5 d^{1} 6 s^{2}$, Th $6 s^{2} 6 p^{6} 6 d^{2} 7 s^{2}$, and $02 s^{2} 2 p^{4}$ ) are treated explicitly in the Hamiltonian of the Schrödinger equation. The generalized gradient approximation [10] was used to approximate the electron exchange and correlation contributions to the total energy. The kinetic energy cut-off for the planewaves was chosen to be $500 \mathrm{eV}$ and the k-point spacing applied was $0.1 \AA^{-1}$, which results in 9 k-points for the $2 \times 1 \times 1$ supercell ( $F m 3 m$ converted to $P 1$ supercell) that was chosen for calculating the energy of a limited number of cation configurations within the binary $\mathrm{ZrO}_{2}-\mathrm{CeO}_{2}-\mathrm{ThO}_{2}$ solid solution. The total energy convergence tolerance was $1 \times 10^{-5} \mathrm{eV} / \mathrm{atom}$. Geometry optimizations were performed without symmetry constraints (P1), and, in order to improve convergence and save computational time, the starting unit cell parameters for the intermediate structures were weighted averages of the end-member parameters. A spinpolarized approach was unnecessary because the cations involved in the solid solution have no unpaired electrons. For the same reason, the Hubbard $U$ approach was not applied, as there were no unpaired $5 \mathrm{f}$ electrons that required screening for erroneous Coulombic interactions.

Cation-cation interaction parameters and Margules parameters were fit to the energies from the $\mathrm{QM}$ calculations and were subsequently used in Monte-Carlo (MC) simulations, in which the system is scaled up to 2048 exchangeable cations [11,12]. The Bogoliubov integration scheme was then used to determine the temperature-dependent Gibbs free energy and configurational entropy [13]. The methodology for the interaction parameter fitting and subsequent MC simulation and thermodynamic integration are detailed in Shuller et al. [7].

All dioxide end members in this study have the isometric fluorite structure, where the cations fill every other cube of the oxygen sublattice (coordination number $=8$ ). In addition to being isometric at high $T, \mathrm{ZrO}_{2}$ is typically monoclinic baddeleyite at room temperature; however, an isometric structure can be stabilized at ambient conditions (e.g., Y-stabilized $\mathrm{ZrO}_{2}$ ) [14]. The $2 \times 1 \times 1$ unit cell contains 8 exchangeable cation sites, and there are multiple configurations at different compositions, resulting in 26 unique (i.e., symmetrically not equivalent) configurations across the binary. For the $2 \times 1 \times 1$ unit cell, four interaction types $\left(J_{1}, J_{2}, J_{3}, J_{5}\right)$ were included in the fit (Table 1 ), where the first interaction type describes nearest-neighbor interactions, the second describes second nearest-neighbor interactions, and so forth. The fourth nearest-neighbor interaction is linearly dependent on the first nearest-neighbor interaction; therefore, $J_{4}$ is not included in the fit.

Calculations were also performed for the solid solutions within the tetragonal framework in order to identify a potentially different solid solution behavior based on crystal symmetry.
The lattice for the tetragonal structure $\left(P 4_{2} / n m c\right)$ was redefined to allow for better comparison with the isometric structure; therefore, the same configurations across the solid solution could be calculated. For the tetragonal system, five interaction types $\left(J_{1 a}, J_{1 b}, J_{2}, J_{3}\right.$, and $\left.J_{5}\right)$ were fit (Table 1 ). Because of the decrease in symmetry, $J_{1}$ from the cubic structure was replaced by $J_{1 a}$ and $J_{1 b}$, i.e., nearest neighbor interactions parallel and perpendicular to the $c$ direction, respectively.

The cation-cation interaction parameters can be fit using two primary methods:

(1) The first method fits a concentration-dependent and configuration-independent energy, $E_{0}$, to the average of excess energies for each concentration, which are subsequently fit to the difference from $E_{0}$ for each configuration using the $J$ formalism. In this case, the enthalpy of mixing from the quantum-mechanical calculations is fit using Eq. 1, where $E_{0}$ is approximated using an asymmetric Margules function with the Margules terms ( $W_{1}$ and $W_{2}$ in Eq. 2$)$ and $\Sigma n_{i} J_{i}$ describes the configuration-dependent energy using interaction parameter $J$.

(2) Alternatively, the interaction parameters can be fit without the Margules parameters.

Together, the procedures provide complementary information. The first method first subtracts the concentration dependent average $E_{0}(x)\left(E_{0}(x)\right.$ is added after the Monte Carlo runs are completed) from the total energy such that only the configuration and temperature dependent part of the excess energy is treated in the Monte-Carlo runs. The Monte Carlo procedure converges to the local cation distribution with the lowest energy for a given temperature without gaining more energy by partially or fully exsolving the system within the large supercell. In contrast, the second procedure, because $E_{0}(x)$ is built into the interaction parameter, $J$, gains further energy if the enthalpy of mixing values are positive for a given concentration, $x$, and lower for concentrations higher and lower than $x$. Thus, the first method forces the system to locally assume the concentration of the overall unit cell, while the second allows for exsolution. In other words, while method 1 provides thermodynamic information on the true solid solution, method 2 allows for the evaluation of the thermodynamics of a potentially locally exsolved system.

$\Delta H_{m i x}=\mathrm{E}_{0}+\Sigma n_{i} J_{i}$

$E_{0}=x(1-x)\left[W_{1} x+W_{2}(1-x)\right]$

For example, Fig. 1a shows the quantum-mechanical results for the isometric $\mathrm{Th}_{x} \mathrm{Zr}_{1-x} \mathrm{O}_{2}$ binary, where the magnitude and

Table 1

Cation-cation exchange fit parameters including Margules parameters $\left(W_{1}\right.$ and $\left.W_{2}\right)$, interaction parameters $\left(J_{i}\right)$, and the correlation coefficient for the fit. The $J$ parameters are defined as by the direction from the cation at $(0,0,0)$ to the cation at the distance/direction described by the interaction. Actual distances depend on the unit cell parameters for the given solid solution. Note a negative $J_{i}$ indicates heterocationic interactions are preferred, while a positive $J_{i}$ indicates homocationic interactions are preferred.

\begin{tabular}{|c|c|c|c|c|c|}
\hline Parameter & $J$ direction & $\mathrm{Th}_{x} \mathrm{Ce}_{1-x} \mathrm{O}_{2}$ & $\mathrm{Ce}_{x} \mathrm{Zr}_{1-x} \mathrm{O}_{2}$ & $\mathrm{Th}_{x} \mathrm{Zr}_{1-x} \mathrm{O}_{2}$ & $\mathrm{Th}_{x} \mathrm{Zr}_{1-x} \mathrm{O}_{2}$ (tetragonal) \\
\hline$W_{1}$ & & 10.1 & 46.7 & 99.3 & 71.1 \\
\hline$W_{2}$ & & 10.5 & 51.9 & 95.7 & 60.9 \\
\hline$J_{1}\left(J_{1 a}\right)$ & {$\left[\begin{array}{lll}1 / 2 & 1 / 2 & 0\end{array}\right]$} & -0.022 & -0.038 & -0.030 & -0.306 \\
\hline$\left(J_{1 b}\right)$ & $\left(\left[\begin{array}{lll}1 / 2 & 0 & 1 / 2\end{array}\right]\right)$ & & & & 0.262 \\
\hline$J_{2}$ & {$[100]$} & 0.009 & -0.027 & -0.085 & 0.129 \\
\hline$J_{3}$ & {$\left[\begin{array}{lll}1 / 2 & 1 & 1 / 2\end{array}\right]$} & 0.007 & 0.021 & 0.033 & -0.022 \\
\hline$J_{4}$ & [110] & \multicolumn{4}{|c|}{ Linearly dependent on $J_{1}$} \\
\hline & {$\left[\begin{array}{lll}1 / 2 & 1 / 2 & 0\end{array}\right]$} & 0.005 & -0.001 & -0.019 & -0.113 \\
\hline Corr coeff & & 0.995 & 0.996 & 0.997 & 0.944 \\
\hline
\end{tabular}




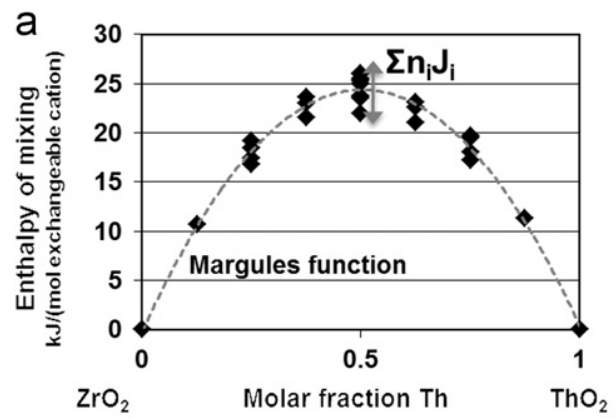

b
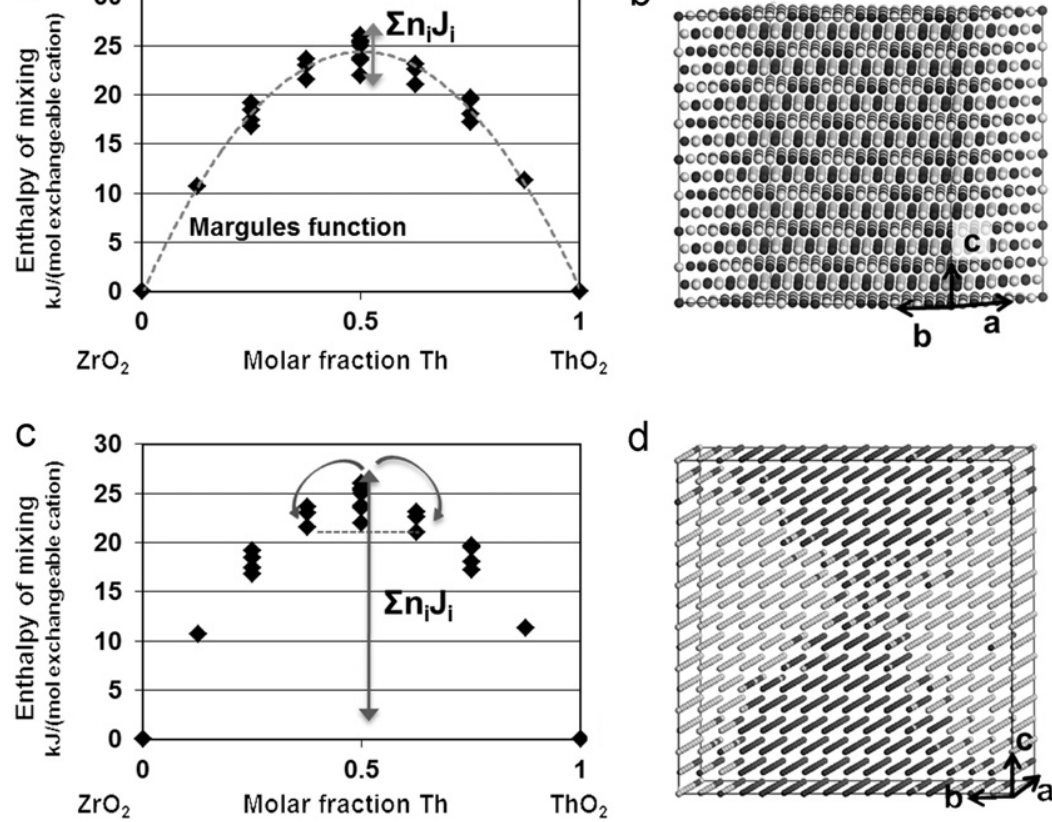

d

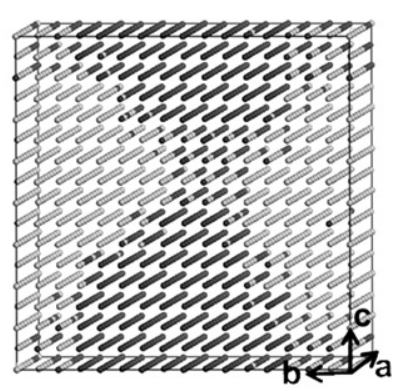

Fig. 1. Enthalpy of mixing from quantum-mechanical calculations fit with (a) the interaction parameters and the Margules parameters results in (b) cation ordering after Monte Carlo simulation. Enthalpy of mixing from quantum-mechanical calculations fit with (c) only interaction parameters results in (d) exsolution into nanodomains.

symmetry are defined by the Margules function, and the spread of the data about that line are defined by the interaction parameters. Such a fit allows the MC simulation to form ordered or exsolved systems; however, it does not force exsolution. Fig. 1b shows cation ordering of the isometric $\mathrm{Th}_{x} \mathrm{Zr}_{1-x} \mathrm{O}_{2}$ binary at $200 \mathrm{~K}$. Alternatively, Fig. 1c shows QM data fit without the Margules function. In this case, the interaction parameters also describe the magnitude of the enthalpy curve above ideality; however, the system exsolves to a lower energy state (i.e., exsolved system) because the interaction parameters describe a larger compositional range of the graph. Thus, the binary readily exsolves, as seen from the formation of nanodomains in the MC simulation (Fig. 1d).

\section{Results}

\subsection{Interaction parameter fit}

For the isometric solid solutions, the interaction parameters for the binaries containing $\mathrm{ZrO}_{2}$ are an order of magnitude larger than those for the $\mathrm{Th}_{x} \mathrm{Ce}_{1-x} \mathrm{O}_{2}$ binary (Table 1 ), indicating that swapping different cations in $\mathrm{ZrO}_{2}$-bearing binaries causes a more significant change in energy than in the $\mathrm{Th}_{x} \mathrm{Ce}_{1-x} \mathrm{O}_{2}$ binary. For example, the nearest-neighbors, second nearest neighbors, and fifth nearest neighbors for the isometric $\mathrm{Th}_{x} \mathrm{Zr}_{1-x} \mathrm{O}_{2}$ binary prefer heterocationic interactions (negative $J$ ), while the third nearest neighbors prefer homocationic interactions (positive $J$ ). The first and second interaction parameters for the tetragonal $\mathrm{Th}_{x} \mathrm{Zr}_{1-x} \mathrm{O}_{2}$ solid solution, which correspond to the first interaction parameter of the isometric solid solution, are an order of magnitude larger than the interaction parameters for the isometric binary. Thus, the tetragonal binary more strongly favors heterocationic interactions in the [110] direction, as compared with the isometric binary. Interestingly, the tetragonal binary exhibits strong homocationic interactions in the [101] or [011] directions, as indicated by the second interaction parameter. The fits for the different binaries are compared in Table 1 . The difference between the Margules parameters, $W_{1}$ and $W_{2}$, for any given solid solution is less than $11 \%$, indicating that the $\mathrm{Th}_{x} \mathrm{Ce}_{1-x} \mathrm{O}_{2}$, and $\mathrm{Th}_{x} \mathrm{Zr}_{1-x} \mathrm{O}_{2}$ binaries are nearly symmetric (i.e., if $W_{1}=W_{2}$ the binary is symmetric). The binaries containing $\mathrm{Zr}$ have higher $\mathrm{E}_{0}$ (larger Margules parameters) and larger interaction parameters (greater temperature dependence of the excess energy, depending on the cation arrangement) than the other solid solutions, indicating a greater tendency for unmixing or cation ordering. In addition, the interaction parameters for the binaries containing $\mathrm{Zr}$ that were fit without the Margules parameters are an order of magnitude greater than those fit with the Margules parameters, and all, except $\mathrm{J}_{9}$ for the tetragonal $\mathrm{Th}_{x} \mathrm{Zr}_{1-x} \mathrm{O}_{2}$, are positive, which indicates that homocationic interactions are favored (Supplemental Data 1).

\subsection{Thermodynamic properties of mixing}

The temperature-dependent thermodynamic properties of mixing, specifically enthalpy of mixing $\left(\Delta H_{\text {mix }}\right)$, Gibbs free energy of mixing $\left(\Delta G_{m i x}\right)$, and configurational entropy of mixing $\left(\Delta S_{\text {mix }}\right)$, were calculated using the Monte-Carlo simulation and thermodynamic integration method described above. The enthalpy of mixing is positive for all binaries; however, the magnitudes of the $\Delta H_{\text {mix }}$ and $\Delta G_{\text {mix }}$ curves, which are indicative of the ideality of the solid solution, vary significantly. For the isometric solid solutions, the maximum enthalpy of mixing, which occurs at a theoretical infinite temperature and $x=0.5$, is only $2.7 \mathrm{~kJ} /(\mathrm{mol}$ exchangeable cations) for $\mathrm{Th}_{x} \mathrm{Ce}_{1-x} \mathrm{O}_{2}$, while the maximum enthalpy of mixing is $11.8 \mathrm{~kJ} /\left(\mathrm{mol}\right.$ exchangeable cations) for $\mathrm{Ce}_{x} \mathrm{Zr}_{1-x} \mathrm{O}_{2}$ and $23 \mathrm{~kJ} /$ (mol exchangeable cations) for $\mathrm{Th}_{x} \mathrm{Zr}_{1-x} \mathrm{O}_{2}$ (Fig. 2). Interestingly, the enthalpy curves for the isometric solid solutions containing $\mathrm{Zr}$ appear to flatten at $x=0.5$ at $100 \mathrm{~K}$ for $\mathrm{Ce}_{x} \mathrm{Zr}_{1-x} \mathrm{O}_{2}$ and $300 \mathrm{~K}$ for $\mathrm{Th}_{x} \mathrm{Zr}_{1-x} \mathrm{O}_{2}$, and the enthalpy curves for the tetragonal $\mathrm{Th}_{x} \mathrm{Zr}_{1-x} \mathrm{O}_{2}$ solid solution have a minimum at $x=0.5$ for temperatures below $1500 \mathrm{~K}$ (Fig. 3).

The Gibbs free energy of mixing curves (Fig. 4), which clearly show miscibility gaps for all binaries, was used to generate the temperature-composition phase diagram for the isometric solid solutions (Fig. 5). A miscibility gap exists for all calculated temperatures (below $3000 \mathrm{~K}$ ) for the isometric and tetragonal $\mathrm{Th}_{x} \mathrm{Zr}_{1-x} \mathrm{O}_{2}$ binary, below $1500 \mathrm{~K}$ for the isometric $\mathrm{Ce}_{x} \mathrm{Zr}_{1-x} \mathrm{O}_{2}$ 


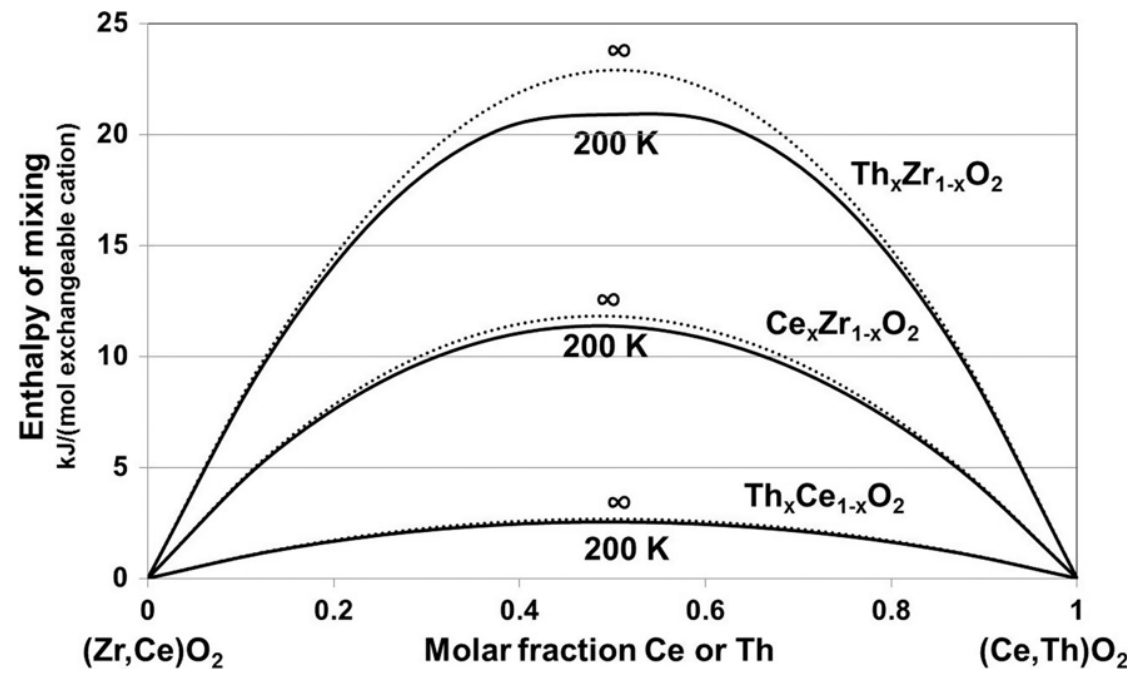

Fig. 2. Enthalpy of mixing for the $\mathrm{Th}_{x} \mathrm{Zr}_{1-x} \mathrm{O}_{2}, \mathrm{Ce}_{x} \mathrm{Zr}_{1-x} \mathrm{O}_{2}$, and $\mathrm{Th}_{x} \mathrm{Ce}_{1-x} \mathrm{O}_{2}$ binaries. $\mathrm{The} \mathrm{Th}_{x} \mathrm{Ce}_{1-x} \mathrm{O}_{2}$ binary is nearly ideal and minimal temperature dependence is observed for all three binaries.

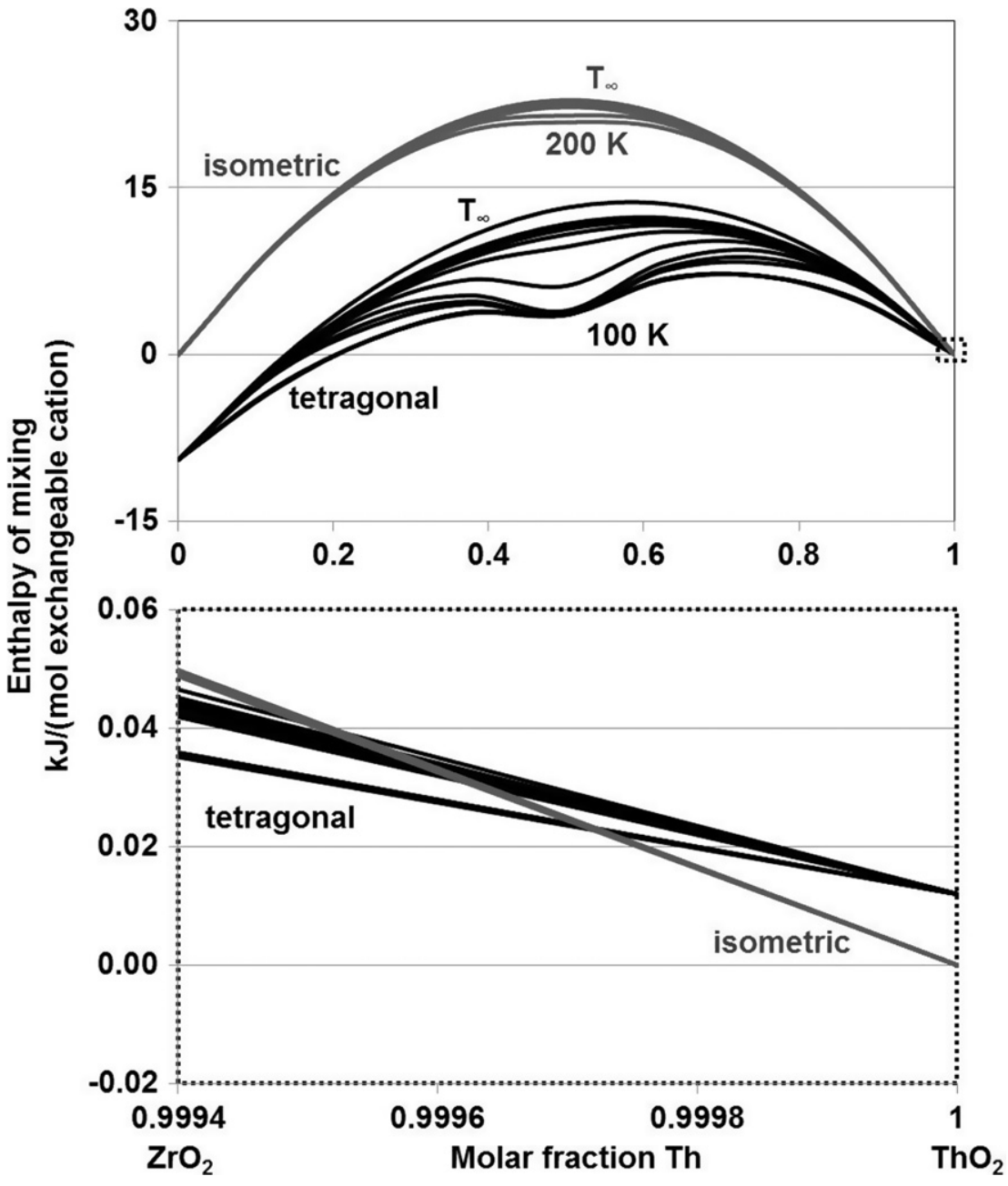

Fig. 3. Enthalpy of mixing for the $\mathrm{Th}_{x} \mathrm{Zr}_{1-x} \mathrm{O}_{2}$ binary with isometric and tetragonal symmetry. All configurations were calculated without symmetry constraints ( $\left.P 1\right)$; the isometric $\mathrm{ZrO}_{2}$ end member remains isometric and the tetragonal $\mathrm{ZrO}_{2}$ end member remains tetragonal; however, the intermediate members tend to vary from isometric to tetragonal across the solid solution. The tetragonal $\mathrm{ThO}_{2}$ end member becomes isometric when calculated with or without symmetry constraints.

binary, and below $600 \mathrm{~K}$ for the isometric $\mathrm{Th}_{x} \mathrm{Ce}_{1-x} \mathrm{O}_{2}$ binary. Thus, exsolution is expected based on the Gibbs free energy of mixing. The configurational entropy curves for the isometric solid solutions are nearly independent of temperature and equal to the point entropy, which is the configurational entropy without ordering (Fig. 6a,c,e). However, for the $\mathrm{Ce}_{x} \mathrm{Zr}_{1-x} \mathrm{O}_{2}$ and $\mathrm{Th}_{x} \mathrm{Zr}_{1-x} \mathrm{O}_{2}$ binaries, the configurational entropy drops significantly at low temperature at $x=0.5$. Similarly, the configurational entropy for 
a

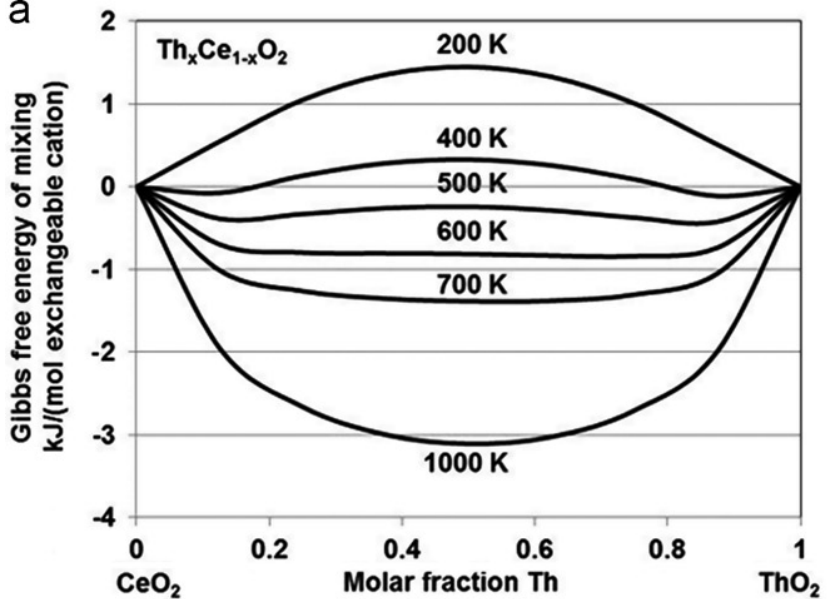

C

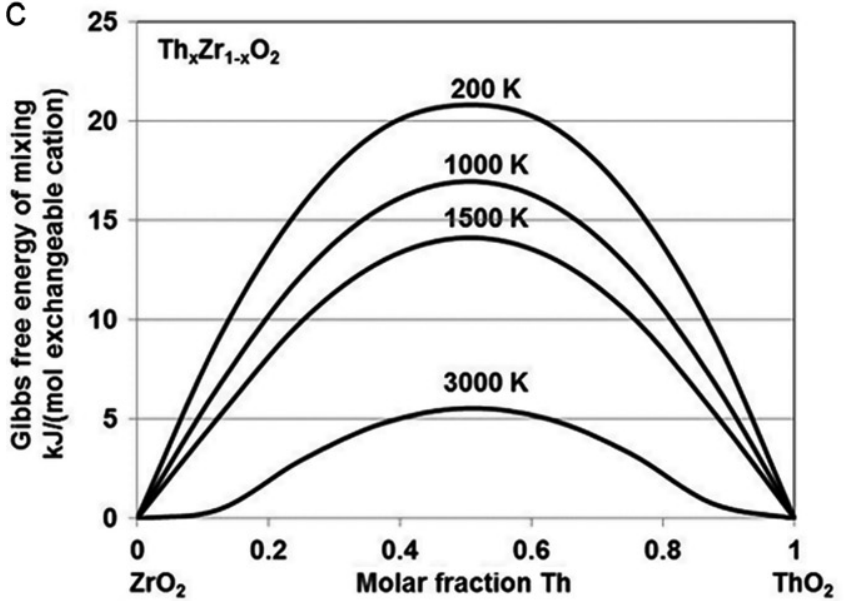

b
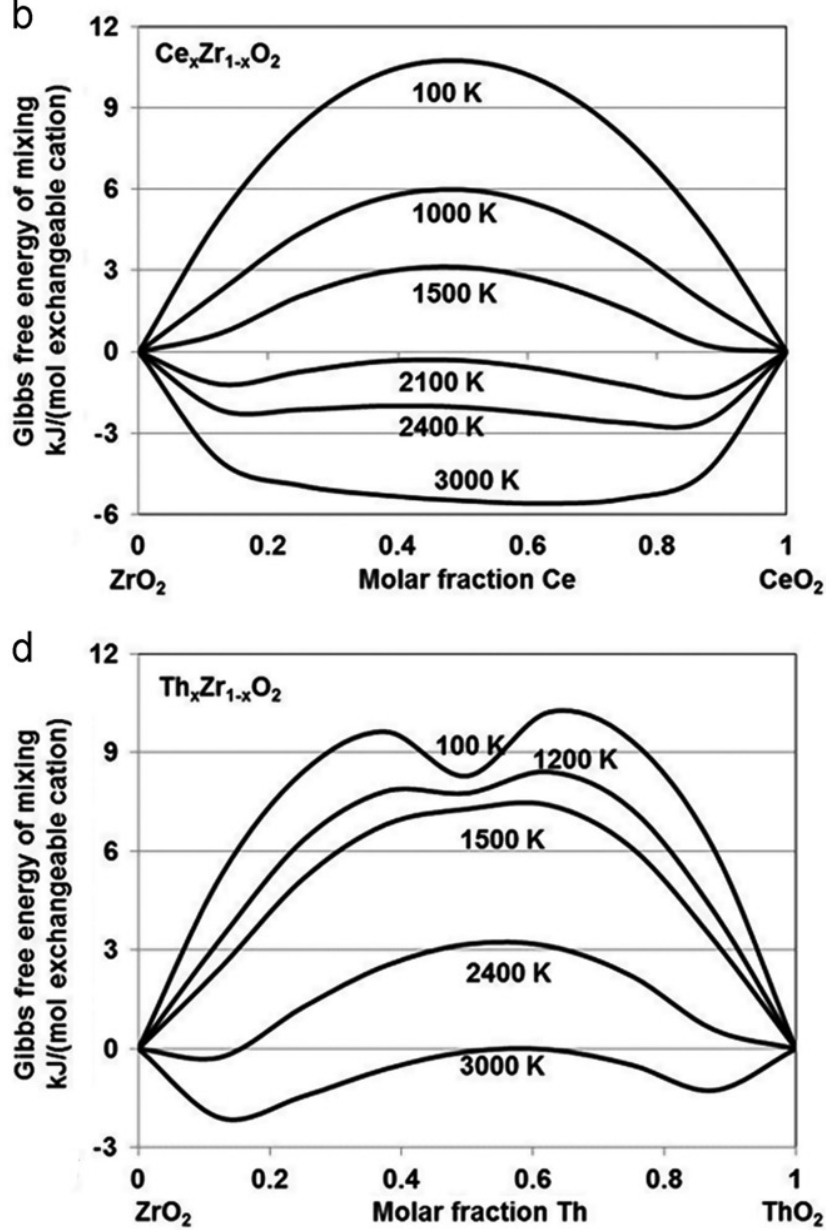

Fig. 4. Gibbs free energy of mixing for (a) isometric $\mathrm{Th}_{x} \mathrm{Ce}_{1-x} \mathrm{O}_{2}$, (b) isometric $\mathrm{Ce}_{x} \mathrm{Zr}_{1-x} \mathrm{O}_{2}$, (c) isometric $\mathrm{Th}_{x} \mathrm{Zr}_{1-x} \mathrm{O}_{2}$, and (d) tetragonal Th $\mathrm{Zr}_{1-x} \mathrm{O}_{2}$. A miscibility gap is expected below $600 \mathrm{~K}$ for isometric $\mathrm{Th}_{x} \mathrm{Ce}_{1-x} \mathrm{O}_{2}, 3000 \mathrm{~K}$ for isometric $\mathrm{Ce}_{x} \mathrm{Zr}_{1-x} \mathrm{O}_{2}$ and $\mathrm{Th}_{x} \mathrm{Zr}_{1-x} \mathrm{O}_{2}$, and at all temperatures evaluated for tetragonal Th $\mathrm{Zr}_{1-x} \mathrm{O}_{2}$.

the tetragonal $\mathrm{ThO}_{2}-\mathrm{ZrO}_{2}$ solid solution has a minimum at $x=0.5$ and decreases significantly below $700 \mathrm{~K}$ for all compositions (Fig. 7). Thus, some cation ordering or exsolution lamellae are expected to form as discussed below.

\section{Discussion}

\subsection{Exsolution}

Although immiscibility is evident for all binaries based on the Gibbs free energy of mixing, the driving force for exsolution for the $\mathrm{Th}_{x} \mathrm{Ce}_{1-x} \mathrm{O}_{2}$ binary may not be great enough to allow the system to actually exsolve. The driving force for exsolution, $E_{\text {exsoln }}$, is defined as the energy difference between the maximum of the free energy curve and the tangent connecting the minima of that curve [7]. In other words, $E_{\text {exsoln }}$ is the energy gained to exsolve the solid solution. The $E_{\text {exsoln }}$ in the context of this work is normalized to the number of cations in the solid solution. A certain amount of the energy is always necessary in order to overcome the interfacial energy between phases that arises from the buildup of strain. The value for $E_{\text {exsoln }}$ is small for the $\mathrm{Th}_{x} \mathrm{Ce}_{1-x} \mathrm{O}_{2}$ binary (only $1.5 \mathrm{~kJ} /(\mathrm{mol}$ cations) at $200 \mathrm{~K}$ ), which is similar to computational and experimental results recently reported for the $\mathrm{Th}_{x} \mathrm{Ce}_{1-x} \mathrm{O}_{2}$ binary [8]. In addition, the $\mathrm{Th}_{x} \mathrm{Ce}_{1-x} \mathrm{O}_{2}$ binary has a Gibbs free energy of mixing similar to that of the $\mathrm{Th}_{x} \mathrm{U}_{1-x} \mathrm{O}_{2}$ binary [7].
While exsolution lamellae were observed at low temperature $(200 \mathrm{~K})$ for the $\mathrm{Th}_{x} \mathrm{U}_{1-x} \mathrm{O}_{2}$ binary, no exsolution lamellae were observed for the $\mathrm{Th}_{x} \mathrm{Ce}_{1-x} \mathrm{O}_{2}$ binary. The lack of exsolution lamellae in the $\mathrm{Th}_{x} \mathrm{Ce}_{1-x} \mathrm{O}_{2}$ binary is attributed to several factors, including kinetic hindrances (i.e., slow cation diffusion and increasing interfacial energy), differences in cation-cation interaction parameters, and challenges in trying to find the appropriate exsolution pathway (see discussion below on cation ordering). The interfacial energy for the $\mathrm{Th}_{x} \mathrm{Ce}_{1-x} \mathrm{O}_{2}$ binary is expected to be slightly greater than that of the Th-U binary because of the difference in ionic radii, and thus, unit cell parameter, is larger $\left(0.19 \AA\right.$ for $\mathrm{Th}_{x} \mathrm{Ce}_{1-x} \mathrm{O}_{2} 0.17 \AA$ for $\left.\mathrm{Th}_{x} \mathrm{U}_{1-x} \mathrm{O}_{2}\right)$. While this difference between the lattice mismatch of the binaries is only $0.02 \AA$, the kinetic hindrances to exsolution, specifically slow cation diffusion, are enhanced at low temperatures, preventing the formation of exsolution lamellae. Such kinetic hindrances are less important in the Monte-Carlo simulations because cation swaps are allowed across kinetic barriers; therefore, they may not be the primary cause for the difference between the $\mathrm{Th}_{x} \mathrm{U}_{1-x} \mathrm{O}_{2}$ and $\mathrm{Th}_{x} \mathrm{Ce}_{1-x} \mathrm{O}_{2}$ simulation results with respect to their ordering tendency. Both binaries have small interaction parameters, yet the sign of the parameters differ for different interaction types for the $\mathrm{Th}_{x} \mathrm{U}_{1-x} \mathrm{O}_{2}$ and $\mathrm{Th}_{x} \mathrm{Ce}_{1-x} \mathrm{O}_{2}$ binaries. The first and fifth nearest neighbor interactions for the $\mathrm{Th}_{x} \mathrm{U}_{1-x} \mathrm{O}_{2}$ binary indicate homocationic interactions ( $\mathrm{U}-\mathrm{U}$ or $\mathrm{Th}-\mathrm{Th}$ ) are favored between nearest neighbors, while the $\mathrm{Th}_{x} \mathrm{Ce}_{1-x} \mathrm{O}_{2}$ binary indicates heterocationic interactions are favored at nearest neighbor distances. Thus, neighboring cations in the $\mathrm{Th}_{x} \mathrm{Ce}_{1-x} \mathrm{O}_{2}$ binary prefer to be different. 

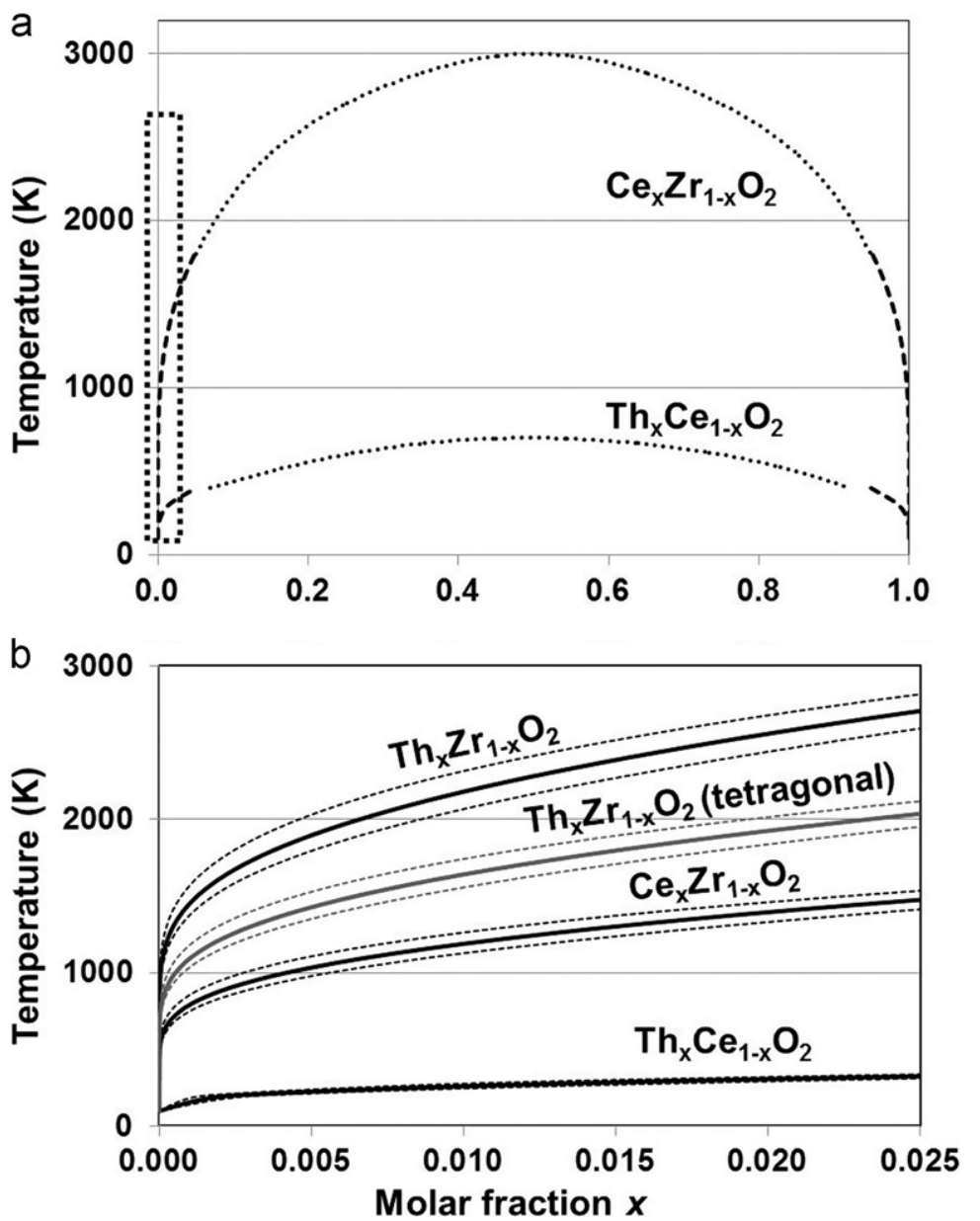

Fig. 5. (a) Full temperature-composition phase diagram showing miscibility gaps for the $\mathrm{Th}_{x} \mathrm{Ce}_{1-x} \mathrm{O}_{2}$ and $\mathrm{Ce}_{x} \mathrm{Zr}_{1-x} \mathrm{O}_{2}$ binaries. The full phase diagram for the $\mathrm{Th}_{x} \mathrm{Zr} \mathrm{r}_{1-x} \mathrm{O}_{2}$ binaries is not shown because solid solutions at molar fractions greater than 0.02 approach the melting temperature for the oxide. The dashed curves are estimated using the Margules approximation for the incorporation limit, while the dotted curves are estimated using the tangential method on the Gibbs free energy of mixing curves for $T=400-600 \mathrm{~K}$ for the $\mathrm{Th}_{x} \mathrm{Ce}_{1-x} \mathrm{O}_{2}$ binary and $T=1800-3000 \mathrm{~K}$ for the $\mathrm{Ce}_{x} \mathrm{Zr}_{1-x} \mathrm{O}_{2}$ binary. (b) Inset shows detail of the low composition range, where the solid solidus is an average of the linear and Margules approximations for the incorporation limit $\left(x_{i n c}\right)$, and the dotted lines representing the solidus are the Margules approximation (above the average) and the linear approximation (below the average).

In addition, the fifth nearest-neighbor interaction for the $\mathrm{Th}_{x} \mathrm{Ce}_{1-x} \mathrm{O}_{2}$ binary is three times less than the same interaction for the $\mathrm{Th}_{x} \mathrm{U}_{1-x} \mathrm{O}_{2}$ binary, indicating that the system has little preference at longer cation distances.

The driving forces for exsolution are much larger for binaries containing $\mathrm{Zr}$, where the driving force for exsolution for the $\mathrm{Ce}_{x} \mathrm{Zr}_{1-x} \mathrm{O}_{2}$ and $\mathrm{Th}_{x} \mathrm{Zr}_{1-x} \mathrm{O}_{2}$ binaries at $T=1000 \mathrm{~K}$ are $E_{\text {exsoln }}=6 \mathrm{~kJ} /$ (mol cations) and $E_{\text {exsoln }}=17 \mathrm{~kJ} /(\mathrm{mol}$ cations), respectively. Such large tendencies for exsolution are expected based on a simple comparison of ionic radii, where the ionic radius of octahedrally-coordinated $\mathrm{Zr}^{4+}$ is only $0.98 \AA$ as compared with the ionic radii of octahedrallycoordinated $\mathrm{Ce}^{4+}$ and $\mathrm{Th}^{4+}$ (1.11 $\AA$ and $1.19 \AA$, respectively). A local strain is imposed on the system when one cation is substituted by another cation that is significantly ( $>10 \%$ ) smaller or larger. To overcome this strain, the system may exsolve into lamellae that are rich in one cation and depleted of the other. Alternatively, the cations may order in such a way so as to reduce the total energy of the binary. The configurational entropy for both the $\mathrm{Ce}_{x} \mathrm{Zr}_{1-x} \mathrm{O}_{2}$ and $\mathrm{Th}_{x} \mathrm{Zr}_{1-x} \mathrm{O}_{2}$ binaries shows a drop in entropy at low $T$ (Fig. 6c,e), which is consistent with either ordering or exsolution.

\subsection{Cation ordering}

Exsolution in the $\mathrm{Th}_{x} \mathrm{Ce}_{1-x} \mathrm{O}_{2}$ binary does not strongly favor any of the three most common planes, that is $\{100\},\{110\}$, or
\{111\}. MC simulations starting with exsolution lamellae parallel to (100), (110), and (111) resulted in relatively homogeneous solid solutions with energies of $-0.161 \mathrm{~kJ} /(\mathrm{mol}$ cations $)$ for lamellae || to (100) and (110) and $-0.157 \mathrm{~kJ} /(\mathrm{mol}$ cations) for lamellae \|| to (111) at $100 \mathrm{~K}$. Thus, even at theoretical temperatures below freezing and initially exsolved cation arrangements, the potential lamellae of the $\mathrm{Th}_{x} \mathrm{Ce}_{1-x} \mathrm{O}_{2}$ binary tend to form a random distribution of cations, resulting in a homogeneous solid solution (Fig. 6b). The homogeneity of the $\mathrm{Th}_{x} \mathrm{Ce}_{1-x} \mathrm{O}_{2}$ solid solution is further confirmed by several experimental measurements $([15,16]$ and references therein) that show the linearity of the change in the unit cell parameters with composition, which is in accordance to Vegard's law.

Alternatively, the configurational entropy for the isometric $\mathrm{Th}_{x} \mathrm{Zr}_{1-x} \mathrm{O}_{2}$ and $\mathrm{Ce}_{x} \mathrm{Zr}_{1-x} \mathrm{O}_{2}$ binaries indicate ordering of the cations in an energetically favored arrangement. This is evident from the significant drop in configurational entropy at low $T$ when $x=0.5$ (Fig. 6c,e). A classic example of cation ordering is the calcite-magnesite $\left(\mathrm{CaCO}_{3}-\mathrm{MgCO}_{3}\right)$ solid solution, which exhibits a significant drop in entropy at $x=0.5$. This drop in entropy corresponds to ordering of the $\mathrm{Ca}$ and $\mathrm{Mg}$ ions into alternating $\mathrm{Ca}$ and $\mathrm{Mg}$ layers in the [001] direction, forming dolomite $\mathrm{CaMg}\left(\mathrm{CO}_{3}\right)_{2}$ [17]. Similarly, cation ordering is observed for isometric $\mathrm{Th}_{x} \mathrm{Zr}_{1-x} \mathrm{O}_{2}$ binary at $200 \mathrm{~K}$ at $x=0.5$, where rows of alternating cations lie in the (100) plane. Since the (100), (010), 


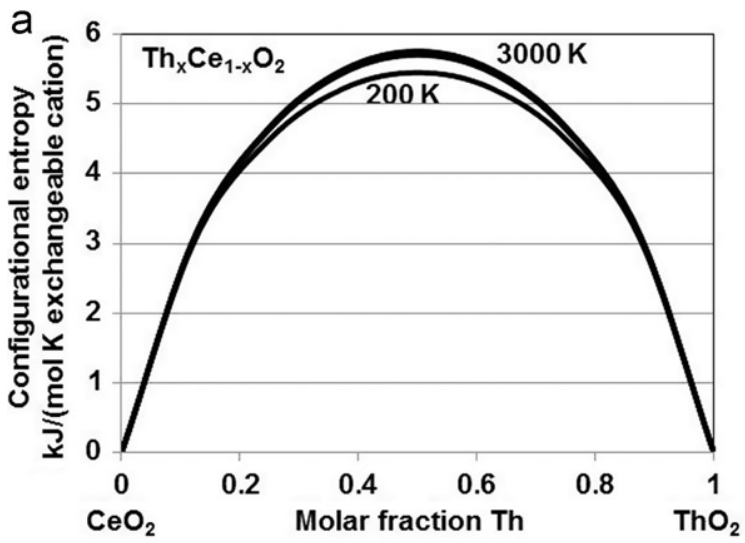

b

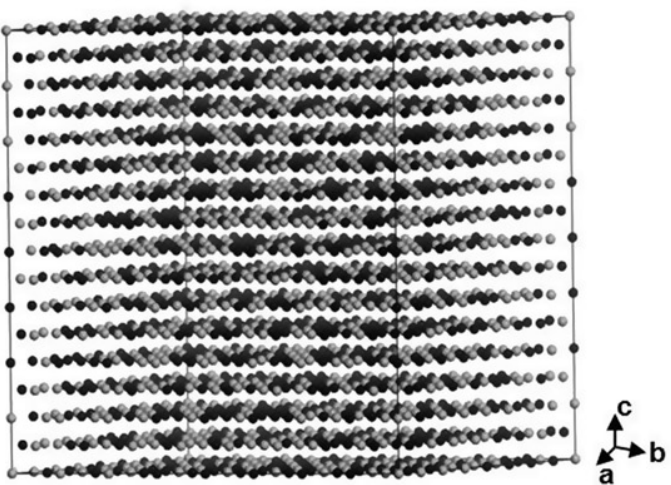

C

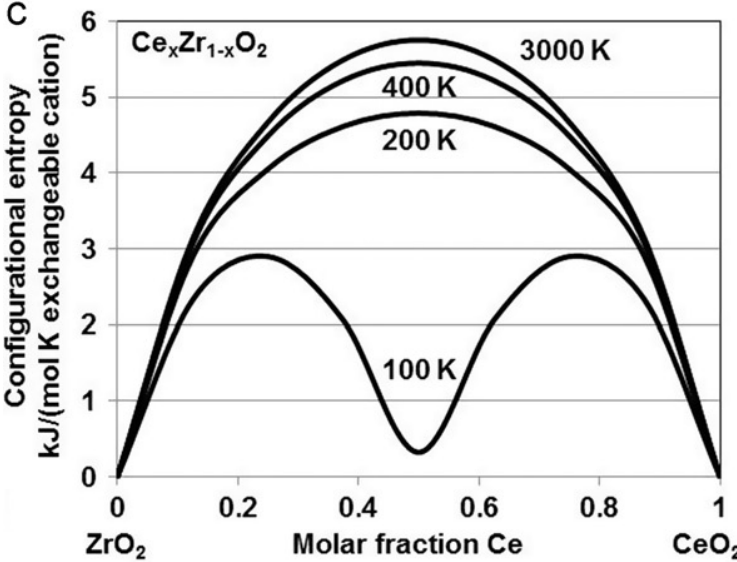

d
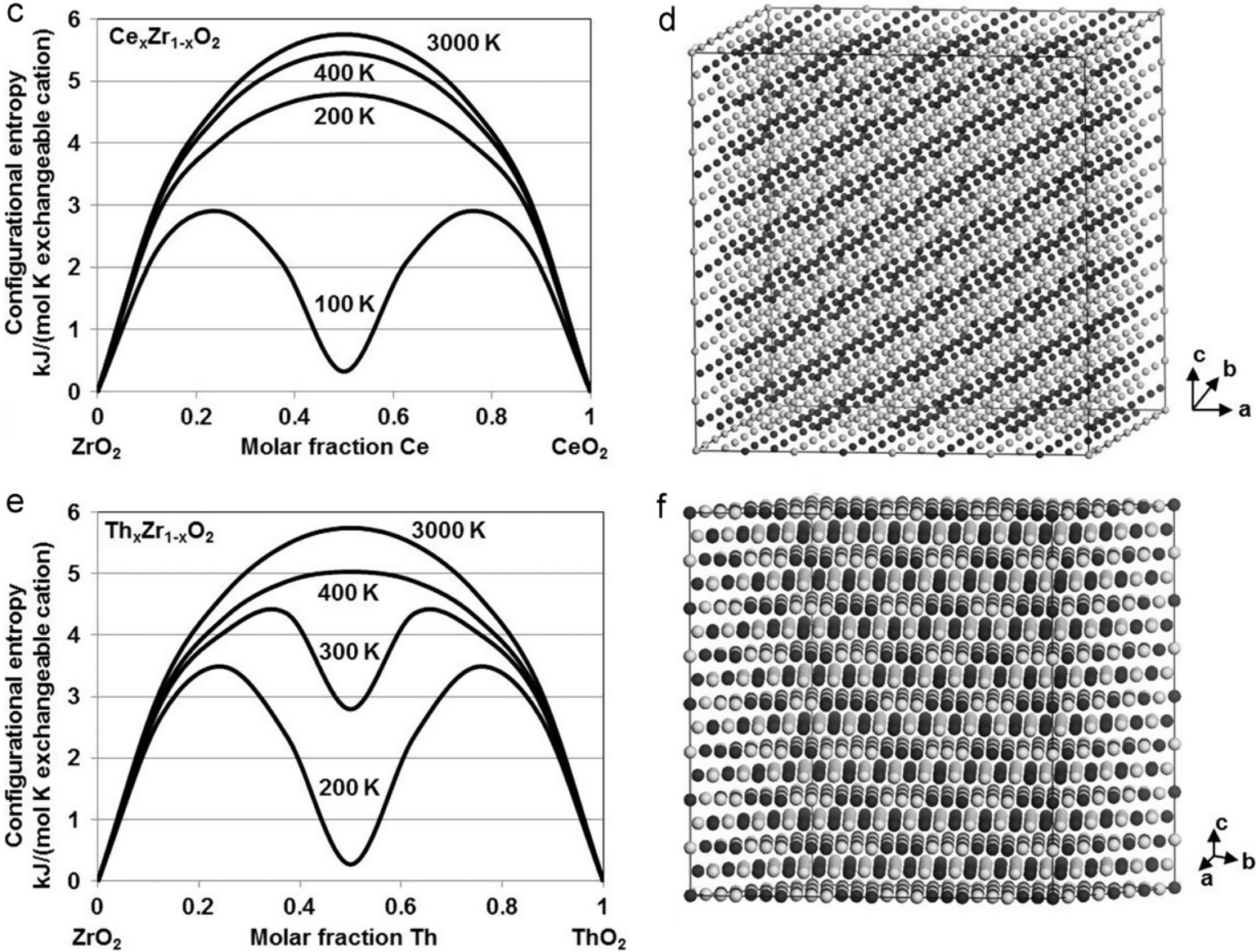

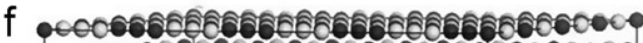
- 00000000000000000000000

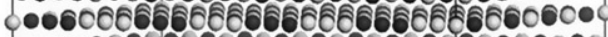
-

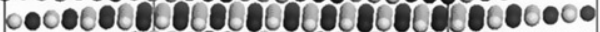
๑ง

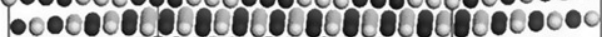
๑ o o 00000000000000000000000000

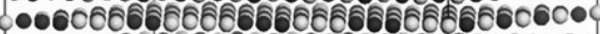
- 00000100000000000000000 - oง bo 0000000000000000000000 -00000600000060000000100000000 $\leftrightarrow$

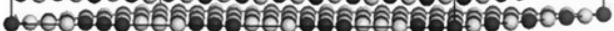

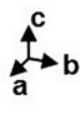

Fig. 6. Configurational entropy curves for (a) isometric $\mathrm{Th}_{x} \mathrm{Ce}_{1-x} \mathrm{O}_{2}$, (c) isometric $\mathrm{Ce}_{x} \mathrm{Zr}_{1-x} \mathrm{O}_{2}$, and (e) isometric $\mathrm{Th}_{x} \mathrm{Zr}_{1-x} \mathrm{O}_{2}$. No ordering is observed for the isometric $\mathrm{Th}_{x} \mathrm{Ce}_{1-x} \mathrm{O}_{2}$ binary (b) because the entropy at all temperatures is nearly equal to the point entropy (i.e., configurational entropy without ordering). However, a sharp minimum at $x=0.5$ at low $T$ is present for the $\mathrm{Zr}$-bearing binaries indicating ordering. (d) The isometric $\mathrm{Ce}_{x} \mathrm{Zr}_{1-x} \mathrm{O}_{2}$ binary displays layering at $100 \mathrm{~K}$ and $x=0.5$ with layers that are about two cation units thick $(\sim 4.5 \AA)$ and $\|$ to $(10 \overline{2})$. (f) The isometric $\operatorname{Th}_{x} \mathrm{Zr}_{1-x} \mathrm{O}_{2}$ binary displays ordering at $x=0.5$ and $T=200 \mathrm{~K}$ with alternating layers $\|$ to $(001)$ of cations with $(i)$ homocationic ordering along $\{110\}$ and heterocationic ordering perpendicular to $\{110\}$ (ii) heterocationic ordering along $\{110\}$ and heterocationic ordering about every three units perpendicular to $\{110\}$.

and (001) planes are equivalent, one would expect domains where ordering changes from one crystallographic direction to another one. In addition, the cation rows lying in subsequent (100) layers along the [001] zone axis are rotated by $30^{\circ}$ (Fig. 6f). However, experimental evidence of an ordered phase in the $\mathrm{Th}_{x} \mathrm{Zr}_{1-x} \mathrm{O}_{2}$ binary has yet to be identified at the nanoscale.

For the isometric $\mathrm{Ce}_{x} \mathrm{Zr}_{1-x} \mathrm{O}_{2}$ binary, nanoscale exsolution lamellae are observed at low $T$ (Fig. 6d), where the lamellae are about two cation layers thick and form $\|$ to $(10 \overline{2})$, which is a linear combination of (101) and $(00 \overline{1})$. Competitive exsolution of lamellae || to any linear combination of the $\{101\}$ and $\{001\}$ may be the cause of the experimentally observed $2.15-4.15 \mathrm{~nm}$ diameter nanocrystallites in $\mathrm{Ce}_{0.5} \mathrm{Zr}_{0.5} \mathrm{O}_{2}$ [18]. Interestingly, single nanocrystallite formation was observed in the present calculations for the $\mathrm{Th}_{x} \mathrm{Zr}_{1-x} \mathrm{O}_{2}$ solid solutions at $x=0.5$ for starting configurations with homocationic interactions parallel to about $\{010\}$ using the approach that allows exsolution (i.e., from interaction parameters fit without Margules parameters; Fig. 1d). The nanoscale exsolution in the $\mathrm{Ce}_{x} \mathrm{Zr}_{1-x} \mathrm{O}_{2}$ binary may alternatively be considered as a superstructure ordering, which is responsible for the decrease in the enthalpy of mixing at $x=0.5$ at $100 \mathrm{~K}$ (Fig. 2). However, further exsolution into thicker lamellae 


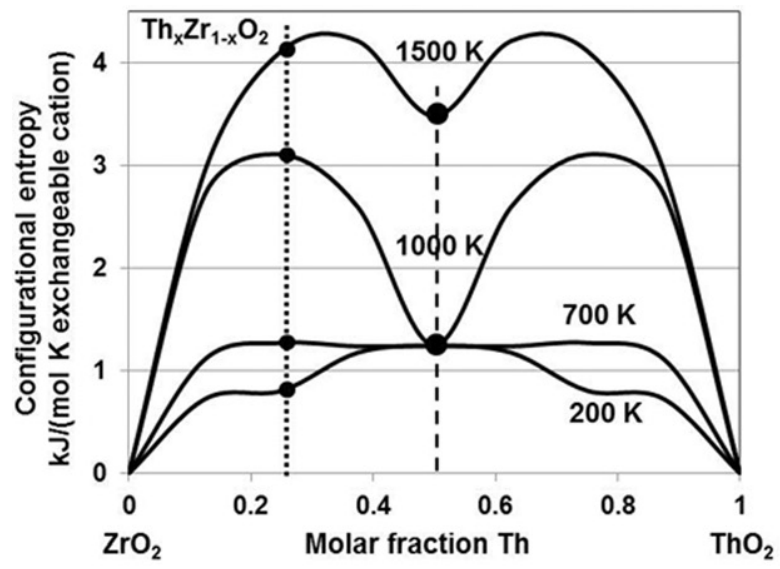

a
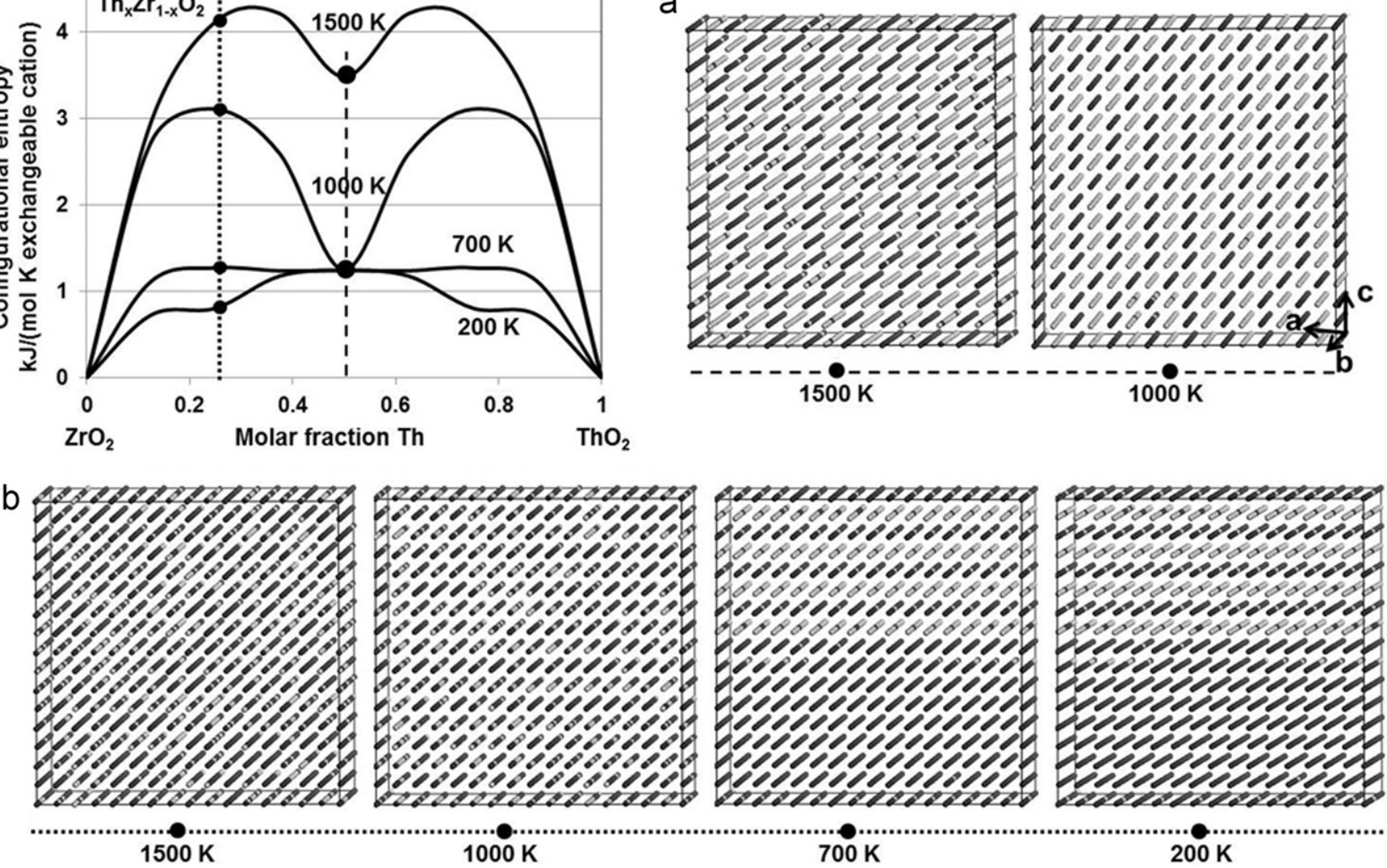

Fig. 7. The configurational entropy for the tetragonal $\mathrm{Th}_{x} \mathrm{Zr}_{1-x} \mathrm{O}_{2}$ binary plateaus below $700 \mathrm{~K}$, which indicates a mixture of ordering and a single phase region for high and low concentrations. The arrangement of cations in (a) and (b) correspond to temperature-concentration points along the tetragonal $\mathrm{Th}_{x} \mathrm{Zr}_{1-x} \mathrm{O}_{2}$ entropy curves. (a) Increased ordering at $x=0.5$ with decreasing temperature from $1500 \mathrm{~K}$ to $1000 \mathrm{~K}$. The cation ordering at temperatures below $1000 \mathrm{~K}$ does not change. (b) No ordering observed for $1000 \mathrm{~K}$ and $1500 \mathrm{~K}$ at $x=0.25$. The plateau at $700 \mathrm{~K}$ and $200 \mathrm{~K}$ results from the combination of a single-phase region and an ordered region at high and low concentrations. The ordered region is consistent with the ordering observed at $x=0.5$, where alternating cation layers are $\|$ to $<001>$.

may be kinetically hindered due to slower cation diffusion, especially at the low temperatures required for the nanoscale exsolution lamellae to form. Experiments have shown that $\mathrm{Ce}_{0.5} \mathrm{Zr}_{0.5} \mathrm{O}_{2}$ synthesized using different methods result in a range of disordered to ordered solid solutions. For example, oxidation of ordered $\mathrm{Ce}(\mathrm{III})_{2} \mathrm{Zr}_{2} \mathrm{O}_{7}$ (pyrochlore) results in disordered $\mathrm{Ce}_{0.5} \mathrm{Zr}_{0.5} \mathrm{O}_{2}$ (fluorite) [19]. Another study showed the formation of three different ordered $\mathrm{Ce}_{0.5} \mathrm{Zr}_{0.5} \mathrm{O}_{2}$ binaries formed using different synthesis precursors and heating cycles [20].

\subsection{End-member stability}

Experimentally, the solid-solution behavior of the $\mathrm{Zr}$-containing binaries is highly dependent on the synthesis method. For example, solid solutions synthesized in a series of three grinding, pelletizing, annealing (at $1200{ }^{\circ} \mathrm{C}, 1300{ }^{\circ} \mathrm{C}$, and $1400^{\circ} \mathrm{C}$ ), and cooling cycles show a miscibility gap between $\mathrm{Th}_{0.05} \mathrm{Zr}_{0.95} \mathrm{O}_{2}$ and $\mathrm{Th}_{0.95} \mathrm{Zr}_{0.05} \mathrm{O}_{2}$ [21]. The phase separations were identified by the appearance of monoclinic zirconia peaks in powder diffraction patterns. However, solid solutions synthesized from a melt (about $3000{ }^{\circ} \mathrm{C}$ ) and quenched show miscibility of $17.5 \mathrm{~mol} \% \mathrm{Th}$ in isometric- $\mathrm{ZrO}_{2}$ [22]. The melting points for $\mathrm{ThO}_{2}, \mathrm{ZrO}_{2}$, and $\mathrm{CeO}_{2}$ are approximately $3300^{\circ} \mathrm{C}$, $2750{ }^{\circ} \mathrm{C}$, and $2600{ }^{\circ} \mathrm{C}$, respectively. Therefore, at small concentrations of $x$, homogeneous cation arrangements in the $\mathrm{Th}_{x} \mathrm{Zr}_{1-x} \mathrm{O}_{2}$ and $\mathrm{Ce}_{x} \mathrm{Zr}_{1-x} \mathrm{O}_{2}$ binaries could be quenched from a melt. However, upon further heat treatment, phase separation occurs due to the transformation of $\mathrm{ZrO}_{2}$ from an isometric to a tetragonal structure. Although the cation coordination is the same in the isometric and tetragonal phases $(C N=8)$, the oxygen atoms are displaced, and the unit cell parameter in the $c$-direction is lengthened for the tetragonal phase. Tetragonal $\mathrm{Ce}_{x} \mathrm{Zr}_{1-x} \mathrm{O}_{2}$ binaries with a range of $c / a$ ratios (1.000-
1.018) have been identified in the compositional ranges 5-20 mol\% and $40-60 \mathrm{~mol} \% \mathrm{CeO}_{2}$ in $\mathrm{ZrO}_{2}$ [23]. In most cases, the tetragonal form is stable at high temperatures and becomes monoclinic at room temperature [24-26]. The phase transition to the monoclinic structure involves a change in the cation coordination from 8 to 7 . The small ionic radius of $\mathrm{Zr}$ requires the shortening of the $\langle\mathrm{Zr}-\mathrm{O}\rangle$ bond distance; therefore, the coordination number decreases due to Coulombic repulsion between the oxygen atoms. Monoclinic $\mathrm{ZrO}_{2}$ $\left(P 2_{1} / c\right)$ is stable at room temperature and occurs in nature as baddeleyite. However, $\mathrm{ThO}_{2}$ and $\mathrm{CeO}_{2}$ are stable at room temperature with the isometric fluorite symmetry $(F m \overline{3} m)$, and occur naturally as the minerals thorianite and cerianite, respectively. Quantum-mechanical calculations confirm the relative stabilities of the fluorite symmetry for $\mathrm{ThO}_{2}$ and $\mathrm{CeO}_{2}$, and baddeleyite symmetry for $\mathrm{ZrO}_{2}$ (Supplemental Data 2).

The structural instability of isometric and tetragonal $\mathrm{ZrO}_{2}$ (or the preference for $\mathrm{ZrO}_{2}$ to be monoclinic) contributes to the driving force for the miscibility gap across most of the isometric and tetragonal $\mathrm{Th}_{x} \mathrm{Zr}_{1-x} \mathrm{O}_{2}$ binary (Fig. $4 \mathrm{c}, \mathrm{d}$ ). Calculations indicate complete miscibility for the isometric $\mathrm{Ce}_{x} \mathrm{Zr}_{1-x} \mathrm{O}_{2}$ binary at high temperature (above $\sim 2000 \mathrm{~K}$ ); however, at high temperature (above $2000 \mathrm{~K}$ ) increased oxygen partial pressures would be necessary to keep $\mathrm{Ce}^{4+}$ oxidized. Tetragonal or isometric zirconia can be stabilized at room temperature with the addition of tetravalent, trivalent or divalent cations (e.g., $\mathrm{CeO}_{2}, \mathrm{Y}_{2} \mathrm{O}_{3}, \mathrm{CaO}$, $\mathrm{MgO})$. Yttria $\left(\mathrm{Y}_{2} \mathrm{O}_{3}\right)$ is the most common trivalent oxide used to stabilize zirconia, where $7-70 \mathrm{~mol} \% \mathrm{Y}_{2} \mathrm{O}_{3}$ stabilizes $\mathrm{ZrO}_{2}$ in the isometric fluorite structure [14], and the solid solution is referred to as yttria-stabilized zirconia (YSZ). While the $\mathrm{CeO}_{2}-\mathrm{ZrO}_{2}$ binary contains a large miscibility gap (Fig. 5 ), the $\mathrm{CeO}_{2}-\mathrm{YSZ}$ binary shows complete miscibility [26]. However, based on a combination of 
Table 2

Comparison of unit cell parameters and bond distances from literature [27] and calculated data. Four configurations at 25 mol\% $\mathrm{CeO}_{2}$ in $\mathrm{ZrO}_{2}$ were calculated, and configuration d is most similar to EXAFS measurements.

\begin{tabular}{|c|c|c|c|c|c|c|c|}
\hline & Measured [27] & Calculated (config. a) & Calculated (config. b) & Calculated (config. c) & Calculated (config. d) & Difference & Difference (config. d only) \\
\hline a $(\AA)$ & 5.169 & 5.193 & 5.280 & 5.196 & 5.171 & $<2.15 \%$ & $0.04 \%$ \\
\hline c $(\AA)$ & 5.253 & 5.323 & 5.303 & 5.313 & 5.255 & $<1.33 \%$ & $0.04 \%$ \\
\hline $\mathrm{c} / \mathrm{a}$ & 1.016 & 1.025 & 1.004 & 1.023 & 1.016 & $<1.17 \%$ & $0.00 \%$ \\
\hline$<\mathrm{Ce}-\mathrm{O}>(\AA)$ & 2.30 & 2.33 & 2.33 & 2.33 & 2.33 & $\sim 1.20 \%$ & $1.20 \%$ \\
\hline$<\mathrm{Zr}-\mathrm{O}>_{1}{ }^{\mathrm{a}}(\AA)$ & 2.10 & 2.07 & 2.09 & 2.08 & 2.05 & $<2.37 \%$ & $2.37 \%$ \\
\hline$<\mathrm{Zr}-\mathrm{O}>2^{\mathrm{a}}(\AA)$ & 2.35 & 2.47 & 2.42 & 2.45 & 2.36 & $<5.04 \%$ & $0.44 \%$ \\
\hline$<\mathrm{Me}-\mathrm{Me}>(\AA)$ & 3.63 & 3.70 & 3.70 & 3.71 & 3.60 & $<2.14 \%$ & $0.88 \%$ \\
\hline
\end{tabular}

${ }^{\text {a }}$ EXAFS data shows two unique $<\mathrm{Zr}-\mathrm{O}>$ distances. Similarly, calculations show distorted $\mathrm{ZrO}_{8}$ octahedra.

XRD and EMPA analyses from the same study, YSZ has minimal solubility in $\mathrm{ThO}_{2}$. Thus, the $\mathrm{Ce}^{4+}$ may be reduced to $\mathrm{Ce}^{3+}$ and cause the stabilization of isometric $\mathrm{ZrO}_{2}$, particularly at high temperatures where the high oxygen partial pressures would be needed to keep $\mathrm{Ce}^{4+}$ oxidized. In addition, the difference in the ionic radii of $\mathrm{Ce}^{4+}$ and $\mathrm{Zr}^{4+}$ has been shown to stabilize tetragonal $\mathrm{ZrO}_{2}[27]$.

The solid-solution calculations presented here were performed without symmetry constraints (P1). Uniaxial elongation was observed in several quantum-mechanical structural optimizations for isometric solid solutions containing $\mathrm{Zr}$, which indicates that the binary is tetragonal. The largest $c / a$ ratio $(c / a=1.011)$ for the isometric $\mathrm{Th}_{x} \mathrm{Zr}_{1-x} \mathrm{O}_{2}$ binary calculations is for the configuration with a concentration $x=0.5$, in which the cations (i.e., Th and $\mathrm{Zr}$ ) exsolve || to $\left(\begin{array}{lll}0 & 0 & 1\end{array}\right)$ and form lamellae that are two cation layers thick. For this configuration, the final unit cell parameter in the $a$ and $b$ directions is $5.334 \AA$, while the final unit cell parameter in the $c$ direction is $5.393 \AA$. The same configuration from the tetragonal $\mathrm{Th}_{0.5} \mathrm{Zr}_{0.5} \mathrm{O}_{2}$ binary and the tetragonal $\mathrm{Ce}_{0.5} \mathrm{Zr}_{0.5} \mathrm{O}_{2}$ binary calculations have $c / a=1.029$ and 1.022 , respectively. Increasing dopant concentrations (i.e., Th or $\mathrm{Ce}$ ) decrease the deviation from the isometric symmetry (i.e., $c / a$ not equal to 1 ) of the binary for the tetragonal binaries calculated, which is in agreement with EXAFS results on $\mathrm{CeO}_{2}$-stabilized tetragonal $\mathrm{ZrO}_{2}$ with $\mathrm{CeO}_{2}$ concentrations of $11 \mathrm{~mol} \%, 16 \mathrm{~mol} \%$, and $25 \mathrm{~mol} \%$ [28]. At $25 \mathrm{~mol} \%$, some isometric peaks appear in the XRD spectra, which may indicate phase separation of cubic- $\mathrm{CeO}_{2}$ from tetragonal- $\mathrm{ZrO}_{2}$. The calculated $\langle\mathrm{Ce}-\mathrm{O}\rangle,\langle\mathrm{Zr}-\mathrm{O}\rangle$, and $<$ Me-Me $>$ bond distances are $\sim 1-2 \%$ of the experimental bond distances for the $25 \mathrm{~mol} \% \mathrm{CeO}_{2}$-tetragonal $\mathrm{ZrO}_{2}$ solid solutions, with the exception of the $\sim 5 \%$ difference for the long $\langle\mathrm{Zr}-\mathrm{O}\rangle$ bond distance (Table 2). Configuration d, for which the Ce atoms lie in the (110) plane, has unit cell parameters and bond distances most similar to the EXAFS measurements. The distortion of the $\mathrm{ZrO}_{8}$ octahedra was observed in the present calculations and compares well with EXAFS results [28]. The lengthening of the $<\mathrm{Ce}-\mathrm{O}>$ bond distance is due to compensation for the larger ionic radius of $\mathrm{Ce}(\mathrm{IV})$ as compared with $\mathrm{Zr}(\mathrm{IV})(1.11 \AA$ and $0.98 \AA$, respectively). The same change in $\langle\mathrm{Me}-\mathrm{O}\rangle$ bond distances is also observed for the $\mathrm{Th}_{x} \mathrm{Zr}_{1-x} \mathrm{O}_{2}$ binaries.

\subsection{Miscibility limit}

Typically, the limit of incorporation is determined based on a tangent line connecting the minima of a Gibbs free energy curve. The $x$ values where the tangent meets the curve are the limits of miscibility. For example, the tangent to the $\mathrm{Th}_{x} \mathrm{Ce}_{1-x} \mathrm{O}_{2}$ curve at $400 \mathrm{~K}$ meets the curve at $x=0.1$ and $0.9 \mathrm{M}$ fraction of $\mathrm{ThO}_{2}$. Thus, based solely on the Gibbs free energy of mixing, $10 \mathrm{~mol} \%$ of $\mathrm{CeO}_{2}$ can be incorporated into $\mathrm{ThO}_{2}$ and $10 \mathrm{~mol} \%$ of $\mathrm{ThO}_{2}$ can be incorporated into $\mathrm{CeO}_{2}$. The minima of Gibbs free energy curves for the $\mathrm{Ce}_{x} \mathrm{Zr}_{1-x} \mathrm{O}_{2}$ and $\mathrm{Th}_{x} \mathrm{Zr}_{1-x} \mathrm{O}_{2}$ binaries are very close to the end members; therefore, a graphical approach, such as the use of a tangential line, is not a reliable estimate of the incorporation limit.

The minima of the Gibbs free energy of mixing for the $\mathrm{Ce}_{x} \mathrm{Zr}_{1-x} \mathrm{O}_{2}$ and $\mathrm{Th}_{x} \mathrm{Zr}_{1-x} \mathrm{O}_{2}$ binaries are beyond the resolution of the calculations. Therefore, an estimate for the incorporation limit can be made based on an approximation of the Gibbs free energy curve, $\Delta G_{m i x}$, which can be written in terms of $\Delta H_{\text {mix }}$ and $\Delta S_{\text {mix }}$ (Eq. 3). $\Delta H_{\text {mix }}$ is approximated with a symmetric Margules function $\left[\Delta H_{m i x}=A x(1-x)\right.$ ], and $\Delta S_{m i x}$ is approximated with the Stirling's approximation $\left[\Delta S_{\text {mix }}=-R\{x \ln x+(1-x) \ln (1-x)\}\right]$. The $A$ parameter is determined based on the enthalpy of mixing at the lowest concentration $x$ calculated. For the quantum-mechanical calculations the lowest concentration is $x=0.125$, while the smallest concentration possible in the Monte-Carlo simulation is $x=0.0005$. This approximation becomes especially useful if only quantum-mechanical calculations are available (e.g., [29]). The condition for the minimum of the $\Delta G_{\text {mix }}$ becomes Eq. 4 , where the first derivative with respect to $x$ of Eq. 3 is set equal to zero. Eq. 5 , which is the solution to Eq. 4, has the form $x=\mathrm{f}(x)$ and can be solved numerically using the Banach fixed point theorem. With a suitable starting value for $x$ on the right hand side, Eq. 5 is adequately solved after a few iterations, such that $x_{n+1, i n c}=x_{n, i n c}$.

$\Delta G_{\text {mix }}(x)=A x(1-x)+R T(x \ln x+(1-x) \ln (1-x))$

$\frac{\partial}{\partial x}[A x(1-x)+R T(x \ln x+(1-x) \ln (1-x))]_{x=x_{\text {inc }}}=0$

$x_{n+1, i n c}=\left(1-x_{n, i n c}\right) \exp \left(\frac{-A\left[1-2 x_{n, i n c}\right]}{R T}\right)$

Alternatively, the limit of incorporation, $x_{i n c}$, can be approximated based on the assumption that the $\Delta H_{\text {mix }}$ curve is linear at low $x$, where $x_{\min }$ is the minimum concentration evaluated in the Monte-Carlo simulation [11,30]. Again, $\Delta G_{\text {mix }}$ is written in terms of $\Delta H_{\text {mix }}$ and $\Delta S_{\text {mix }}$, where the $\Delta H_{\text {mix }}$ term is described by a linear approximation, i.e., $\Delta H_{\text {mix }}(x)=\left(\Delta H_{1} / x_{1}\right) x$. The solution to the minimum of the $\Delta G_{\text {mix }}$, based on the linear approximation of $\Delta H_{m i x}$, is an analytical expression (Eq. 6), where $\Delta H_{1}$ is the enthalpy of mixing corresponding to a specific temperature $T$ and concentration $x_{1}$.

$x_{i n c}=\frac{e^{\left(-\Delta H_{1} / x_{1}\right)(1 / R T)}}{\left(1+e^{\left(-\Delta H_{1} / x_{1}\right)(1 / R T)}\right)}$

The estimated $x_{i n c}$ can be used to generate a temperaturecomposition phase diagram (Fig. 5). The phase diagram shows the composition $x_{\text {inc }}$ versus temperature based on an average of the $x_{i n c}$ estimated by the Margules and the linear approximations. The dotted line above the average is the result from the Margules approximation, while the dotted line below the average is the 
result from the linear approximation. The calculated miscibility limits are significantly lower than those measured experimentally. For example, at $1400{ }^{\circ} \mathrm{C}(1673 \mathrm{~K})$, Grover and Tyagi [21] found $5 \mathrm{~mol} \% \mathrm{ZrO}_{2}$ in $\mathrm{ThO}_{2}$ and $20 \mathrm{~mol} \% \mathrm{ZrO}_{2}$ in $\mathrm{CeO}_{2}$, based on the refinement of unit cell parameters from powder X-ray diffraction patterns across the solid-solution. However, the calculated miscibility limit of $\mathrm{ZrO}_{2}$ in $\mathrm{ThO}_{2}$ and $\mathrm{CeO}_{2}$ at $1673 \mathrm{~K}$ is 0.25 and $3.98 \mathrm{~mol} \%$, respectively. Incorporation limits increase from 0.25 to $1.09 \%$ when calculated for the tetragonal $\mathrm{Th}_{x} \mathrm{Zr}_{1-x} \mathrm{O}_{2}$ binary. Similar discrepancies between calculated and measured miscibility limits were noted for solid solutions with zircon, e.g., $\mathrm{Me}_{x} \mathrm{Zr}_{1-x} \mathrm{SiO}_{4}$, where $\mathrm{Me}=\mathrm{Ce}, \mathrm{Hf}, \mathrm{Th}, \mathrm{U}$, and $\mathrm{Pu}$ [30]. Both computational and experimental limitations are noted as the sources of possible error. Computationally, the limited number of configurations evaluated quantum-mechanically may be insufficient to capture the complexity of long-range cation-cation interactions. Experimentally, solid solutions may not have reached thermodynamic equilibrium. In addition, solid solutions may be exsolved on the nanoscale, such as the $\mathrm{Ce}_{0.5} \mathrm{Zr}_{0.5} \mathrm{O}_{2}$ nanocrystallite exsolution [18] described above.

\section{Conclusions}

The thermodynamic properties of mixing, specifically the enthalpy of mixing $\left(\Delta H_{\text {mix }}\right)$, Gibbs free energy of mixing $\left(\Delta G_{\text {mix }}\right)$, and configurational entropy $\left(\Delta S_{\text {mix }}\right)$, were calculated for the $\mathrm{Th}_{x} \mathrm{Ce}_{1-x} \mathrm{O}_{2}, \mathrm{Ce}_{x} \mathrm{Zr}_{1-x} \mathrm{O}_{2}$, and $\mathrm{Th}_{x} \mathrm{Zr}_{1-x} \mathrm{O}_{2}$ binaries across the complete solid solution within an isometric framework $(\mathrm{Fm} \overline{3} \mathrm{~m})$ and $\mathrm{Th}_{x} \mathrm{Zr}_{1-x} \mathrm{O}_{2}$ was also calculated within the tetragonal framework $\left(\mathrm{P}_{2} / \mathrm{nmc}\right)$. As determined experimentally, the Th-Ce binary forms a complete solid solution, and no cation ordering or nanoscale exsolution was observed. Below room temperature at $x=0.5$, nanoscale exsolution with lamellae $\|$ to $(10 \overline{2})$ are observed for the $\mathrm{Ce}_{x} \mathrm{Zr}_{1-x} \mathrm{O}_{2}$ binary. At the same conditions, a complex cation-ordering scheme is observed for the Th- $\mathrm{Zr}$ binary. Exsolution is expected for the solid solutions containing $\mathrm{Zr}$ based on the large miscibility gap observed in the Gibbs free energy of mixing, as well as the instability of isometric and tetragonal $\mathrm{ZrO}_{2}$ at room temperature. However, $\mathrm{ZrO}_{2}$ does have limited solubility for both $\mathrm{CeO}_{2}$ and $\mathrm{ThO}_{2}$. Calculated estimates of the miscibility limit within the $\mathrm{Ce}_{x} \mathrm{Zr}_{1-x} \mathrm{O}_{2}$ and $\mathrm{Th}_{x} \mathrm{Zr}_{1-x} \mathrm{O}_{2}$ binaries are significantly lower than measured limits. Results from the tetragonal $\mathrm{Th}_{x} \mathrm{Zr}_{1-x} \mathrm{O}_{2}$ binary solid solution show decreased enthalpy of mixing due to increased stability of tetragonal $\mathrm{ZrO}_{2}$. Thus, the discrepancy between measured and calculated estimates of miscibility may be reduced by further calculations in which the $\mathrm{ZrO}_{2}$ end-member is considered to be monoclinic $\left(P 2_{1} / c\right)$, which is the most stable form at room temperature. Further analysis of the complete range of $\mathrm{Ce}_{x} \mathrm{Zr}_{1-x} \mathrm{O}_{2}$ and $\mathrm{Th}_{x} \mathrm{Zr}_{1-x} \mathrm{O}_{2}$ solid solutions within a monoclinic framework is necessary in order to understand the impact of the structural stability on the solid-solution properties of these binaries.

\section{Acknowledgments}

The authors acknowledge support from the US Department of Energy, Nuclear Energy University Program grant (DE-AC0705ID14517).

\section{Appendix A. Supporting information}

Supplementary data associated with this article can be found in the online version at http://dx.doi.org/10.1016/j.jssc.2012.08.033.

\section{References}

[1] M. Kazimi, Amer. Sci. 91 (2003) 408.

[2] IAEA, IAEA-TECDOC-1155 (2000)

[3] E.C. Subbarao, in: A.H. Heuer, L.W. Hobbs (Eds.), Adv. Ceram., American Ceramic Society, Ohio, 1981, pp. 1-24.

[4] M. Yashima, M. Kakihana, M. Yoshimura, Solid State Ion. 86-8 (1996) 1131-1149.

[5] J. Kaspar, P. Fornasiero, M. Graziani, Catal. Today 50 (1999) 285-298.

[6] C. Degueldre, J.M. Paratte, J. Nucl. Mater. 274 (1999) 1-6.

[7] L.C. Shuller, R.C. Ewing, U. Becker, J. Nucl. Mater. 412 (2011) 13-21.

[8] T.Y. Shvareva, V. Alexandrov, M. Asta, A. Navrotsky, J. Nucl. Mater. 419 (2011) $72-75$

[9] M.D. Segall, P.J.D. Lindan, M.J. Probert, C.J. Pickard, P.J. Hasnip, S.J. Clark, M.C. Payne, J. Phys. -Condens. Matter 14 (2002) 2717-2744.

[10] J.P. Perdew, K. Burke, M. Ernzerhof, Phys. Rev. Lett. 77 (1996) 3865-3868.

[11] E.D.A. Ferriss, E.J. Essene, U. Becker, Eur. J. Mineral. 20 (2008) 745-755.

[12] M. Reich, U. Becker, Chem. Geol. 225 (2006) 278-290.

[13] E.R. Myers, Phys. Chem. Miner. 25 (1998) 465-468.

[14] P. Duwez, F.H. Brown, F. Odell, J. Electrochem. Soc. 98 (1951) 356-362.

[15] A.A. Bukaemskiy, D. Barrier, G. Modolo, J. Alloys and Compd. 485 (2009) 783-788.

[16] J.G. Pepin, G.J. McCarthy, J. Am. Ceram. Soc. 64 (1981) 511-516.

[17] A. Navrotsky, C. Capobianco, Am. Miner. 72 (1987) 782-787.

[18] E. Mamontov, R. Brezny, M. Koranne, T. Egami, J. Phys. Chem. B 107 (2003) 13007-13014.

[19] R.G. Haire, P.E. Raison, Z. Assefa, J. Nucl. Sci. Tech. Suppl. 3 (2002) 616-619.

[20] Y. Nagai, T. Yamamoto, T. Tanaka, S. Yoshida, T. Nonaka, T. Okamoto, A. Suda, M. Sugiura, Catal. Today 74 (2002) 225-234.

[21] V. Grover, A.K. Tyagi, J. Nucl. Mater. 305 (2002) 83-89.

[22] P. Duwez, E. Loh, J. Am. Ceram. Soc. 40 (1957) 321-324.

[23] G. Vlaic, R. Di Monte, P. Fornasiero, E. Fonda, J. Kaspar, M. Graziani, J. of Catal. 182 (1999) 378-389.

[24] S. Otsuka-Yao-Matsuo, T. Yao, T. Omata, High Temp. Mater. Process. 22 (2003) 157-164.

[25] G. Vlaic, R. Di Monte, P. Fornasiero, E. Fonda, J. Kaspar, M. Graziani, Catal. Automot. Pollut. Control IV 116 (1998) 185-195.

[26] M. Yashima, H. Arashi, M. Kakihana, M. Yoshimura, J. Am. Ceram. Soc. 77 (1994) 1067-1071.

[27] V. Grover, P. Sengupta, A.K. Tyagi, Mater. Sci. Eng. B-Solid State Mater. for Adv. Technol. 138 (2007) 246-250.

[28] P. Yi, I.W. Chen, J.E. Penner-Hahn, J. Am. Ceram. Soc. 77 (1994) 1281-1288

[29] L.C. Shuller, R.C. Ewing, U. Becker, J. Nucl. Mater. (2011)

[30] E.D.A. Ferriss, R.C. Ewing, U. Becker, Am. Mineral 95 (2010) 229-241. 


\title{
Thermodynamic mixing properties of the $\mathrm{UO}_{2}-\mathrm{HfO}_{2}$ solid solution: density functional theory and Monte Carlo simulations
}

\author{
Ke Yuan ${ }^{\mathrm{a}}$, Rodney C. Ewing ${ }^{\mathrm{a}, \mathrm{b}, \mathrm{c}}$, Udo Becker ${ }^{\mathrm{a}}$ \\ ${ }^{a}$ Department of Earth and Environmental Sciences, University of Michigan, Ann Arbor, MI, \\ 48109, USA; ${ }^{\mathrm{b}}$ Department of Nuclear Engineering and Radiological Sciences, University of \\ Michigan, Ann Arbor, MI, 48109, USA; ' Department of Materials Science and Engineering, \\ University of Michigan, Ann Arbor, MI, 48109, USA
}

\begin{abstract}
$\mathrm{HfO}_{2}$ is a neutron absorber and has been mechanically mixed with $\mathrm{UO}_{2}$ in nuclear fuel in order to control the core power distribution. During the nuclear fission, the temperature at the center of the fuel pellet can reach above $1300 \mathrm{~K}$, where hafnium may substitute uranium and form the binary solid solution of $\mathrm{UO}_{2}-\mathrm{HfO}_{2} \cdot \mathrm{UO}_{2}$ adopts cubic fluorite structure, but $\mathrm{HfO}_{2}$ can occur in monoclinic, tetragonal and cubic structures. Thus, the influence of the $\mathrm{UO}_{2}-\mathrm{HfO}_{2}$ binary on the thermal conductivity and melting point of the fuel will vary as a function of its atomic structure and composition. However, experimental data on the $\mathrm{UO}_{2}-\mathrm{HfO}_{2}$ binary are limited. The enthalpy, free energy, and entropy of mixing of the $\mathrm{UO}_{2}-\mathrm{HfO}_{2}$ binary with three different structure frameworks were calculated using density functional theory and Monte Carlo simulations based on the scheme of thermodynamic integration. The calculated $\mathrm{UO}_{2}-\mathrm{HfO}_{2}$ binary forms extensive solid solution across the entire compositional range but has various exsolution phenomena in different structures. As the structure of the $\mathrm{HfO}_{2}$ end member adopt lower symmetry and become less similar to the cubic $\mathrm{UO}_{2}$, the miscibility gap region on the phase diagram expanded, accompanied by increase in cell volume up to $10 \%$. Close to the $\mathrm{UO}_{2}$ end member, which is relevant to the nuclear fuel, uranium-rich solid solutions exsolve as the fuel cools, and there is a tendency to form the monoclinic hafnium-rich phase in the matrix of isometric uranium-rich solid solution.
\end{abstract}

\section{Introduction}

Neutron absorbers are used in fuel rods in order to depress the power level of freshly loaded nuclear fuel $\mathrm{UO}_{2}$ and to permit higher loading of fuel to achieve longer core life [1]. Neutron poison materials can be coated surrounding fuel pellets, doped into the cladding materials or mixed with $\mathrm{UO}_{2}[2]$. Incorporation of burnable neutron poison materials into the fuel can level 
the power distribution such that power is generated across the core region rather than produced only close to the small area where control rods have been removed. Hafnium is a neutron absorber which has five stable isotopes, ${ }^{176} \mathrm{Hf}$ (natural isotopic abundance 5.2\%, thermal capture cross section 23 barns), ${ }^{177} \mathrm{Hf}\left(18.6 \%, 373\right.$ barns), ${ }^{178} \mathrm{Hf}\left(27.1 \%, 84\right.$ barns), ${ }^{179} \mathrm{Hf}(13.7 \%, 41$ barns), and ${ }^{180} \mathrm{Hf}$ (35.2\%, 13 barns). The removal of one isotope of hafnium by absorption of neutrons leads to the production of another neutron absorber, and continues through a chain of five absorbers. Among all $\mathrm{Hf}$ isotopes, ${ }^{177} \mathrm{Hf}$ has the highest cross section, and further transmutation to other isotopes will decrease the neutron cross sections. Therefore, enriching hafnium in ${ }^{177} \mathrm{Hf}$ will lead to a lower residual reactivity burden than that of hafnium with natural isotopic ratio, and experimental research has been conducted to investigated the potential benefits of using ${ }^{177} \mathrm{Hf}$ as the burnable neutron poison where ${ }^{177} \mathrm{HfO}_{2}$ was mixed with $\mathrm{UO}_{2}$ to make homogenously distributed ${ }^{177} \mathrm{Hf}$ fuel pellets [1].

During the fission of the fuel, the temperature at the center of the fuel pellets can reach above $1300 \mathrm{~K}$ [3], where $\mathrm{Hf}$ may disperse into the atomic structure of $\mathrm{UO}_{2}$ to form a solid solution, and the $\mathrm{UO}_{2}-\mathrm{HfO}_{2}$ binary with different structures could exit. $\mathrm{UO}_{2}$ has the cubic fluorite structure, but $\mathrm{HfO}_{2}$ exists in three different phases at ambient pressure. At room temperature, $\mathrm{HfO}_{2}$ exists in the baddeleyite monoclinic structure, which is the most stable phase. At about $2000 \mathrm{~K}, \mathrm{HfO}_{2}$ undergoes the phase transition to the tetragonal phase, and at around $2900 \mathrm{~K}$, tetragonal hafnia transforms to the high-temperature cubic fluorite structure [4-10]. As a result, the influence of the $\mathrm{UO}_{2}-\mathrm{HfO}_{2}$ binary solid solution on the thermal conductivity and melting temperature of the fuel depends both on the atomic structure and composition of the $\mathrm{UO}_{2}-\mathrm{HfO}_{2}$ binary. The thermodynamic mixing properties of the $\mathrm{UO}_{2}-\mathrm{HfO}_{2}$ solid solution were calculated and the phase diagram of the $\mathrm{UO}_{2}-\mathrm{HfO}_{2}$ binary was derived. In addition, exsolution phenomena, the energetics of cation ordering and the similarity to the $\mathrm{UO}_{2}-\mathrm{ZrO}_{2}$ binary were investigated.

\section{Methods}

\subsection{Calculation of total energy}

Energy calculations are based on density functional theory (DFT) using the CASTEP program. The generalized gradient approximation (GGA) with Perdew and Wang's 1991 (PW91) was used for the electron exchange-correlation energy. In addition, the excess energies (equation 5) 
obtained using GGA-PW91 with ultrasoft pseudopotentials for core electrons of U, Hf, and O, which are the parameters used for current calculations, were compared with results using GGAPBE, on the fly pseudopotentials, and less than $7 \%$ difference was found. Configurations with different Hf-U ratios were calculated within the monoclinic, tetragonal, and cubic frameworks without symmetry constraints. A $2 \times 1 \times 1$ supercell was used for all structures, each containing 8 exchangeable cation sites for U-Hf substitution (Fig1). Spin polarization was included in the calculation for the configurations containing $\mathrm{U}^{4+}$ cations. All the spins of $\mathrm{U}^{4+}$ cations have the same orientation in order to avoid any additional effect from possible spin ordering. The planewave cut-off energy was $500 \mathrm{eV}$ and $9 \mathrm{k}$-points were used for all calculations.
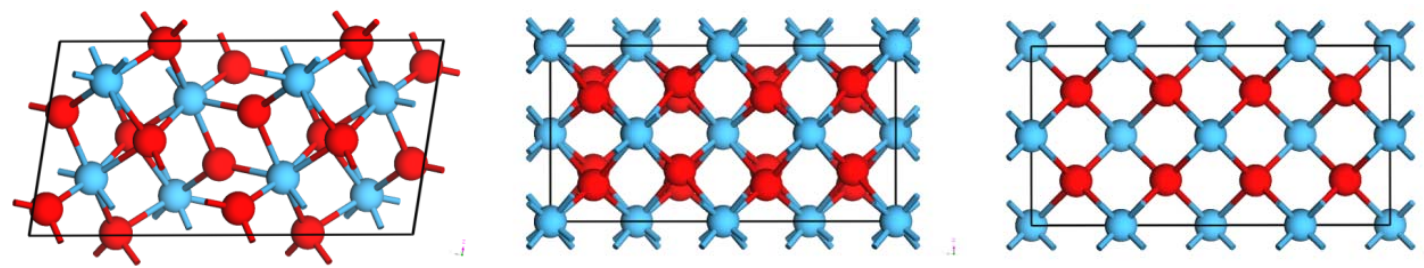

Fig1. $2 \times 1 \times 1$ supercell of monoclinic $\left(P 2_{1} / C\right.$, left $)$, tetragonal $\left(P 4_{2} / n m c\right.$, middle) and cubic (Fm3m, right) $\mathrm{HfO}_{2}$, projections along [010]. Red balls and blue balls denote $\mathrm{O}$ and $\mathrm{Hf}$ atoms, respectively.

\subsection{Fitting of the cation exchange parameters}

After geometry optimization of a number of different configurations (about 25 for each structure types), a series of ground-state energies at $0 \mathrm{~K}$ were obtained from the CASTEP calculations. The excess energy of mixing of the different $\mathrm{UO}_{2}-\mathrm{HfO}_{2}$ solid solutions was determined by using equation 1 .

$$
E_{\text {excess }}=E\left(U_{x} H f_{1-x} O_{2}\right)-x E\left(U O_{2}\right)-(1-x) E\left(H f O_{2}\right)
$$

The excess energy term is defined as the differences between the energies of a certain cation configuration (CASTEP energy) from the mechanical mixture of the end members with the same concentration. The energy related to the cation exchange can be extracted from the total lattice energy calculated for many different configurations. The $J$ formalism replaces the energies of mixing obtained from geometry optimizations using quantum-mechanical calculations by defining a set of effective pair energies, the $J$. It permits the expression of the excess energy of a 
solid solution as a combination of the excess energy terms related to pairs of interacting atoms [11-15]. In general, the excess total energy of mixing can be expressed as equation 2, taking into account separate pairs of interactions for two cations, $U$ and Hf respectively.

$$
E_{\text {excess }}=E_{0}+\sum_{i}\left(n_{U-H f}^{i} E_{U-H f}^{i}+n_{U-U}^{i} E_{U-U}^{i}+n_{H f-H f}^{i} E_{H f-H f}^{i}\right)
$$

Where $i$ indicates the type of neighboring interaction between pairs of cations (i.e., first, second, and third nearest-neighbor interactions), $n$ represents the number of cation-cation interactions for each type, and $E$ is the interaction energy between U-Hf, U-U, and Hf-Hf. The individual cation interaction energies $E$ can be combined into a single term $J$ called the cation exchange potential in equation 3.

$$
J_{U-H f}^{i}=E_{U-H f}^{i}-\frac{1}{2}\left(E_{U-U}^{i}+E_{H f-H f}^{i}\right)
$$

For simulating solid solutions using the cation interaction model, cation interaction energies $\left(E_{U \text { - }}\right.$ $\left.{ }_{U}, E_{H f-H f} E_{U-H f}\right)$ are not independent of the chemical composition. Therefore, $E_{0}$ in equation 2 is a concentration-dependent, but not configuration dependent term that is used to capture the asymmetry of the excess energy curves. $E_{0}$ is approximated by a Margules function in equation 4.

$$
E_{0}=x(1-x)\left[m_{1} x+m_{2}(1-x)\right]
$$

The energy of mixing can be fit by the following equation:

$$
E_{\text {excess }}=E_{0}+\sum_{i} n_{U-H f}^{i} J_{U-H f}
$$

The calculated excess energy of mixing were fit by both Margules terms and cation exchange potential $J$ (U-Hf) $[12,13]$. First $\left(J_{1}\right)$, second $\left(J_{2}\right)$, and third $\left(J_{3}\right)$ nearest neighbor interactions of U-Hf were considered in the fitting (Table 1).

Table1. Margules terms $\left(m_{1}, m_{2}\right)$ and cation exchange parameters $J_{i}(\mathrm{~kJ} / \mathrm{mol})$ in cubic, tetragonal and monoclinic frameworks of the $\mathrm{UO}_{2}-\mathrm{HfO}_{2}$ solid solution.

\begin{tabular}{ccccccc}
\hline Structure & $m_{1}$ & $m_{2}$ & $J_{1}$ & $J_{2}$ & $J_{3}$ & $R^{2}$ \\
\hline & & & & & & \\
cubic & 8.736 & 1.784 & 0.0078 & -0.0249 & -0.0023 & 0.975
\end{tabular}




\begin{tabular}{ccccccc} 
tetragonal & 7.229 & 2.922 & -0.0007 & 0.0154 & -0.0006 & 0.945 \\
monoclinic & 8.021 & 6.462 & 0.0075 & -0.0236 & 0.0047 & 0.974 \\
\hline
\end{tabular}

\subsection{Monte Carlo simulation and thermodynamic integration}

In the Monte Carlo simulation, the Markov chain was generated as follows. For each swapping attempt, two cations in the $8 \times 4 \times 4$ supercell are randomly switched. The cation exchange parameters $J_{i}$ obtained from fitting can be used to calculate the excess energy of mixing of different configurations. The probability of whether to accept the new configuration can be determined by either of the two criteria. (1) If the new energy is lower than the previous one, then accept the new configuration. (2) If the energy is higher than the previous one, using the following equation 6 to calculate the probability, and if the probability is greater than a random number between 0 and 1, then the new configuration is accepted for the next swap.

$$
P=e^{\frac{-\Delta E_{S w a p}}{k_{B} T}}
$$

Where $k_{B}$ is the Boltzmann factor and $T$ is the temperature in Kelvin. The calculations follow an annealing process from $3000 \mathrm{~K}$ to $298 \mathrm{~K}$.

Thermodynamic integration computes the difference of the free energy of the system between the reference state and the state of interest. The free energy expression is equation 7, in which the free energy of interest $F$ can be calculated by integration from the reference states (completely disordered structure) with free energy $F_{0}$ to state of interest at certain temperature and composition by continuously change the parameter $\lambda$ from 0 to 1 . The role of Monte Carlo is to calculate the average free energy of mixing $\langle H\rangle$ at each step of $\lambda$ with reasonable accuracy but without sampling the complete set of configurations [16].

$$
F=F_{0}+\int_{0}^{1}<H>_{\lambda} d \lambda
$$

Calculation of the total excess entropy of mixing can be derived directly from standard thermodynamics by using equation 8 .

$$
\Delta S_{\text {mixing }}=\frac{\Delta H_{\text {mixing }}-\Delta G_{\text {mixing }}}{T}
$$




\section{Results}

\subsection{Comparison of experimental and calculated cell parameters}

The calculated unit cell parameters of the end members all have larger values than the experimental results. The calculated cell parameters give small deviation of $0.7 \%$ for cubic $\mathrm{UO}_{2}$. For $\mathrm{HfO}_{2}$, which has three different structures, the deviations of cell parameters vary from $1.9 \%$ to $4.5 \%$ in a relatively large scale. This may be due to some of the hafnia structures are hightemperature phases, whereas the calculations were conducted at $0 \mathrm{~K}$. The calculated lattice parameters decrease approximately linearly with the increase in Hf concentration in both cubic and monoclinic frameworks (Fig 1, a, c). For the tetragonal framework, lattice parameter $a$ adopts the linearly decreasing trend, but parameter $c$ has a nonlinear relationship with the $\mathrm{Hf}$ mole fraction (Fig 1, b). The cell volume increases by $7-10 \%$ as the structure changes from cubic to monoclinic (Fig 1, d).

Table 2. Comparison between calculated and measured unit cell parameters of $\mathrm{UO}_{2}$ (cubic) [17] and $\mathrm{HfO}_{2}$ (cubic, tetragonal and monoclinic) [18-20].

\begin{tabular}{|c|c|c|c|c|}
\hline lattice parameters & $a(\AA)$ & $b(\AA)$ & $c(\AA)$ & $\beta\left(^{\circ}\right)$ \\
\hline \multicolumn{5}{|l|}{ cubic $\mathrm{UO}_{2}$} \\
\hline Measured & 5.4682 & & & \\
\hline Calculated & 5.508 & & & \\
\hline Deviation (\%) & 0.73 & & & \\
\hline \multicolumn{5}{|l|}{ cubic $\mathrm{HfO}_{2}$} \\
\hline Measured & 5.115 & & & \\
\hline Calculated & 5.257 & & & \\
\hline Deviation (\%) & 2.77 & & & \\
\hline \multicolumn{5}{|l|}{ tetragonal $\mathrm{HfO}_{2}$} \\
\hline Measured & 5.14 & & 5.25 & \\
\hline Calculated & 5.265 & & 5.348 & \\
\hline Deviation (\%) & 2.43 & & 1.87 & \\
\hline
\end{tabular}


monoclinic $\mathrm{HfO}_{2}$

\begin{tabular}{lllll} 
Measured & 5.1156 & 5.1722 & 5.2948 & 99.18 \\
Calculated & 5.333 & 5.406 & 5.467 & 99.78 \\
Deviation (\%) & 4.25 & 4.52 & 3.25 & 0.60 \\
\hline
\end{tabular}
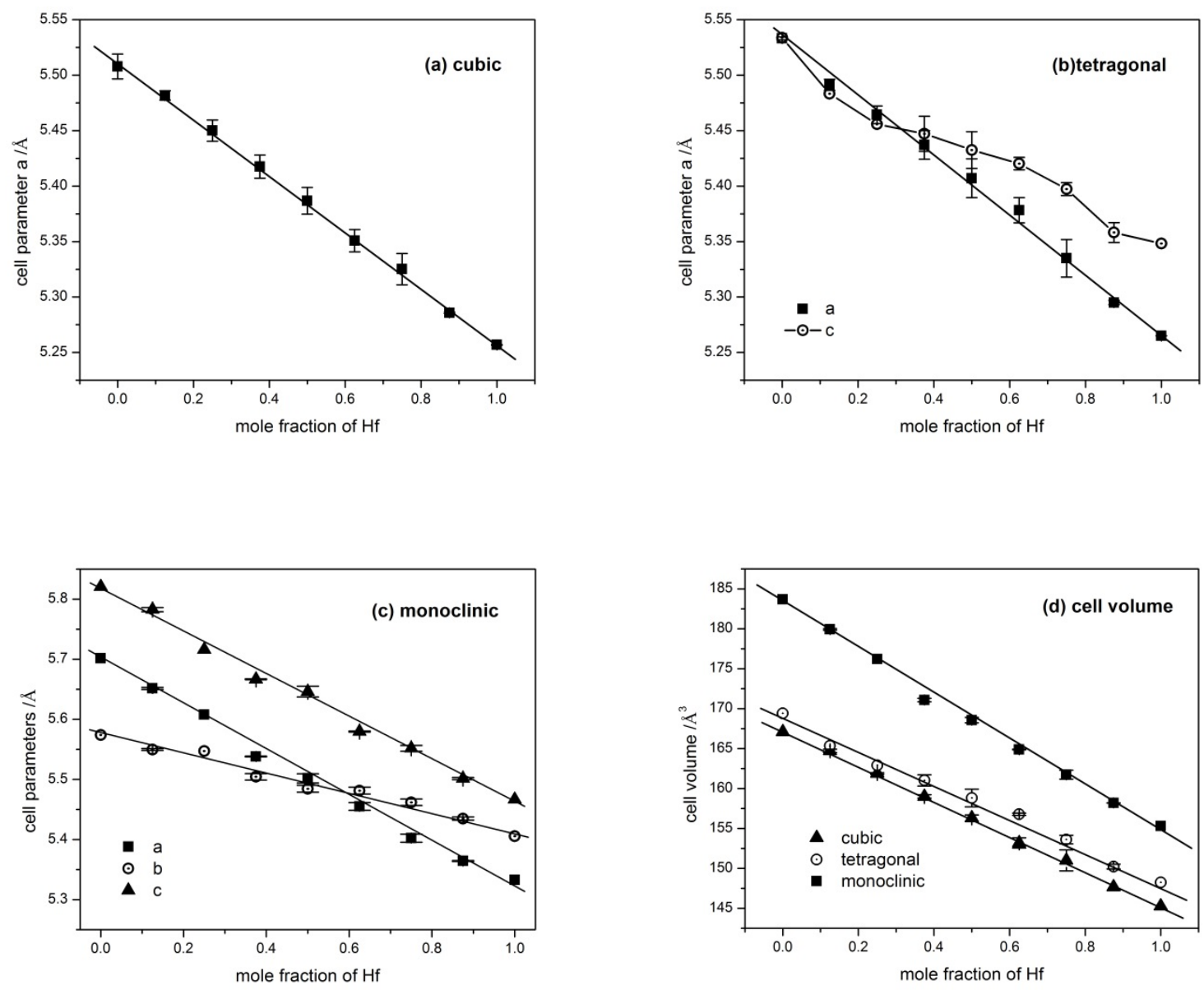

Fig1. Calculated cell parameters as a function of the $\mathrm{Hf}$ mole fraction of the $\mathrm{UO}_{2}-\mathrm{HfO}_{2}$ solid solution in (a) cubic, (b) tetragonal, (c) monoclinic frameworks and (d) cell volume change. Each point is the average value from the same composition, and the error bars represent the standard deviation.

\subsection{Enthalpy of mixing}


The enthalpy of mixing curves becomes less symmetric as the structures adopt higher symmetry (cubic, tetragonal, monoclinic, Fig 2). For the cubic series, the peak value of the enthalpy of mixing is at the Hf mole fraction of around 0.67 which shifted towards 0.5 for tetragonal and monoclinic structures.

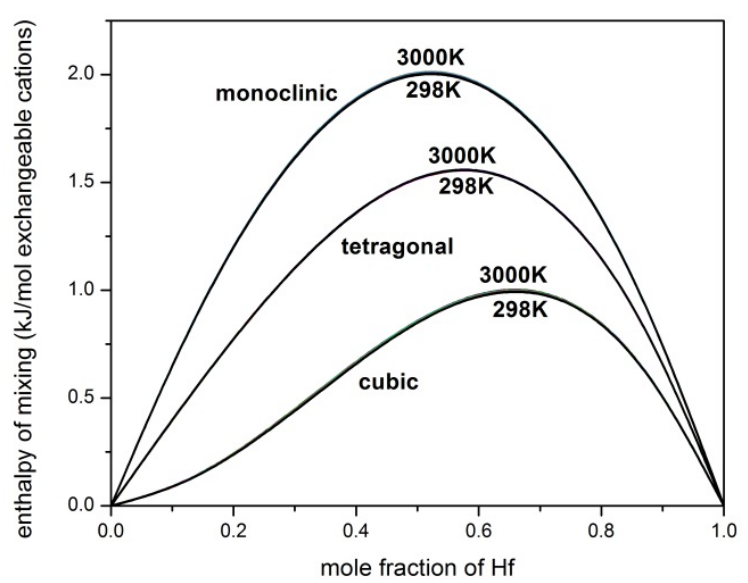

Fig2. Enthalpy of mixing of the $\mathrm{UO}_{2}-\mathrm{HfO}_{2}$ solid solution calculated in cubic, tetragonal and monoclinic frameworks with respect to end members adopted the corresponding structures as 0 enthalpy of mixing.

\subsection{Entropy of mixing}

Entropies of mixing obtained were within $\pm 0.2 \mathrm{~J} / \mathrm{K} \cdot \mathrm{mol}$ exchangeable cations difference of the configurational entropy of mixing and were nearly independent of temperature in all three frameworks. 


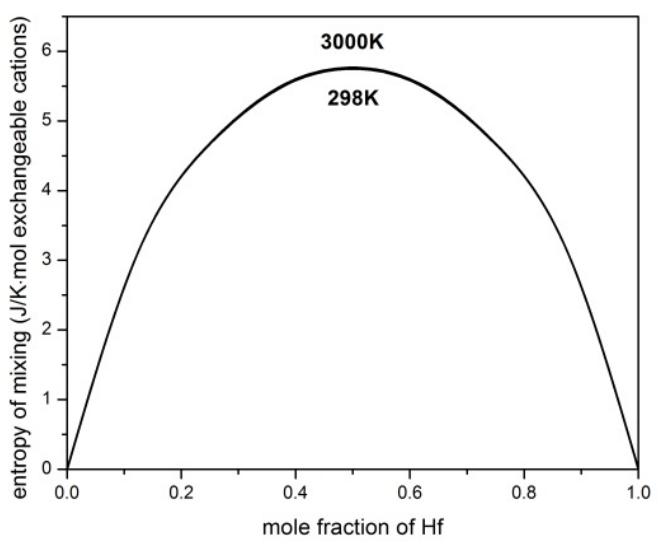

Fig3. Entropy of mixing of the $\mathrm{UO}_{2}-\mathrm{HfO}_{2}$ solid solution

\subsection{Gibbs free energy of mixing}

When both end members $\mathrm{UO}_{2}$ and $\mathrm{HfO}_{2}$ were calculated in cubic framework, negative free energies of mixing was found across the whole temperature range, indicating the mixing is energetically favorable (Fig4. a). When end members were cubic $\mathrm{UO}_{2}$ and tetragonal $\mathrm{HfO}_{2}$, two sets of free energies of mixing curves intersect with each other, where the common tangent lines touched with two points on the free energies of mixing curves of the same temperature, indicating exsolve into two stable phases (Fig4. b). In the cubic and monoclinic case, large energy differences was found for both end members (Fig4. c).
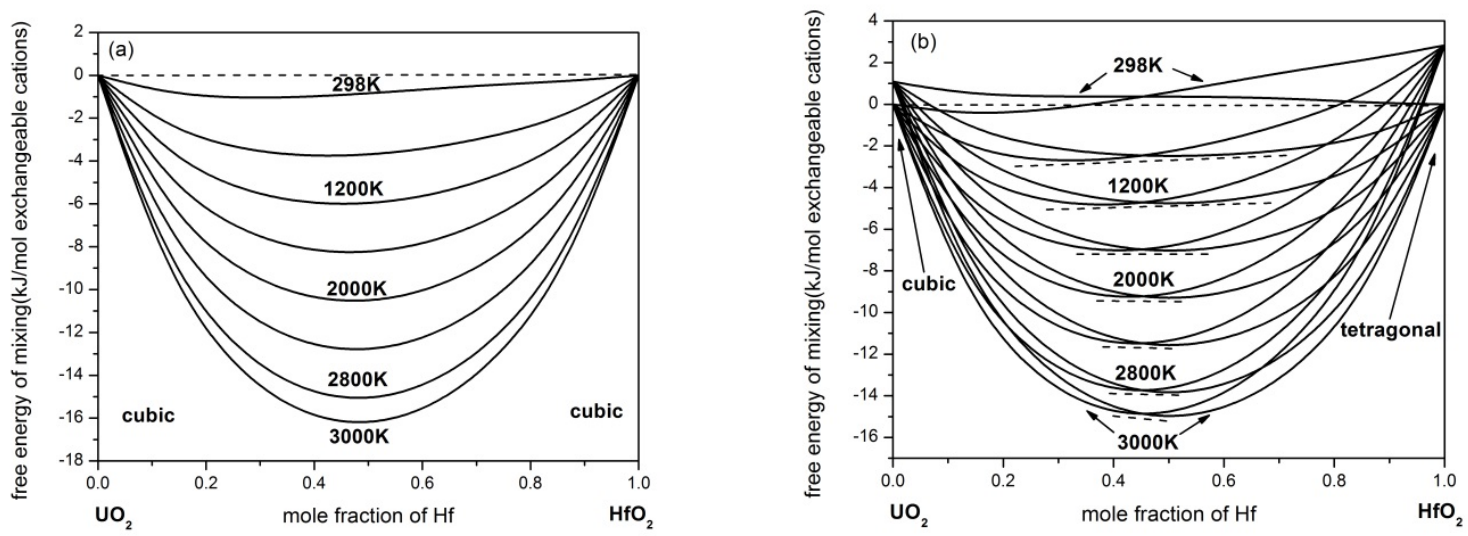


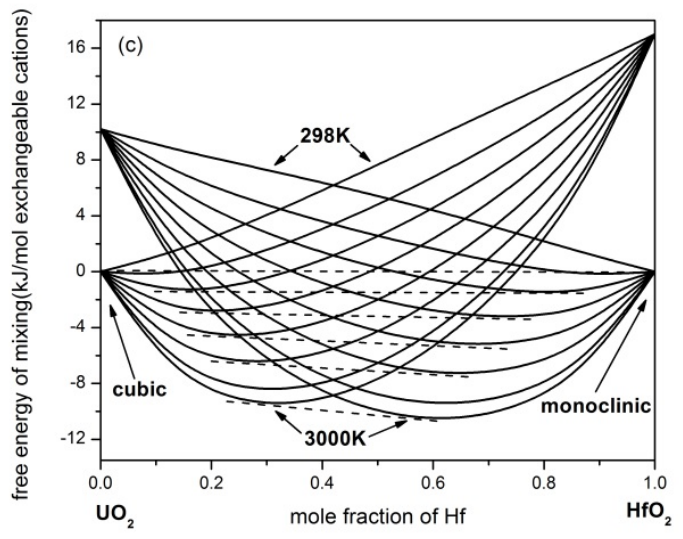

Fig4. Free energy of mixing curves calculated with respect to combinations of cubic-cubic (a), cubic-tetragonal (b) and cubic-monoclinic (c) end members of $\mathrm{UO}_{2}$ and $\mathrm{HfO}_{2}$. Dashed lines are the common tangent line of the free energy of mixing curves of the same temperature for two structures.

\section{Discussion}

\subsection{Cation exchange parameters and ordering}

The cation exchange parameter $\left(J_{2}\right)$ obtained for three structures all have relatively larger absolute values than $J_{1}$ and $J_{3}$ (Table 1), which indicates the second nearest neighbor interaction between $\mathrm{U}$ and $\mathrm{Hf}$ is the dominant interactions. Previous reported cation exchange parameters for similar cation interactions such as $\mathrm{U}$ and $\mathrm{Zr}$ are $J_{I}=0.484 \mathrm{~kJ} / \mathrm{mol}, J_{2}=-0.061 \mathrm{~kJ} / \mathrm{mol}$ and $J_{3}=-0.166$ $\mathrm{kJ} / \mathrm{mol}$ in the zircon structure [21]. Positive values of $J$ mean U-Hf avoidance is preferred (equation 3). Thus, formation of U-Hf interactions in the structure will raise the total energy. As U-Hf interactions are energetically unfavorable to form, clustering of similar cations will be preferred result in increase of the number of U-U and Hf-Hf interactions [22]. In contrast, when $J$ is negative, formation of the U-Hf interaction is favorable. It is possible for the structure to form ordered layers of $\mathrm{U}$ and $\mathrm{Hf}$ cations, which tend to maximize the number of U-Hf interactions leading to the decrease of the total energy. Since first, second and third nearest neighbor cation interactions are included in the fitting, combined effects from three types of interaction energies should be considered. Although some of the $J$ values are negative, entropy of mixing results did not show any tendency towards ordering at various temperatures (Fig 3). Clustering of similar 
cations was visually found in the cubic structure in the lowest energy configuration (Fig 5). The nano-clusters in the $\mathrm{UO}_{2}-\mathrm{ThO}_{2}$ binary indicates that nano-exsolution is more energetically favorable than the exsolved lamellar structure, which can be explained as the exsolution of the lamellae composed of the same cations requires temperatures that are too low for diffusion to actually occur $[21,23,24]$.
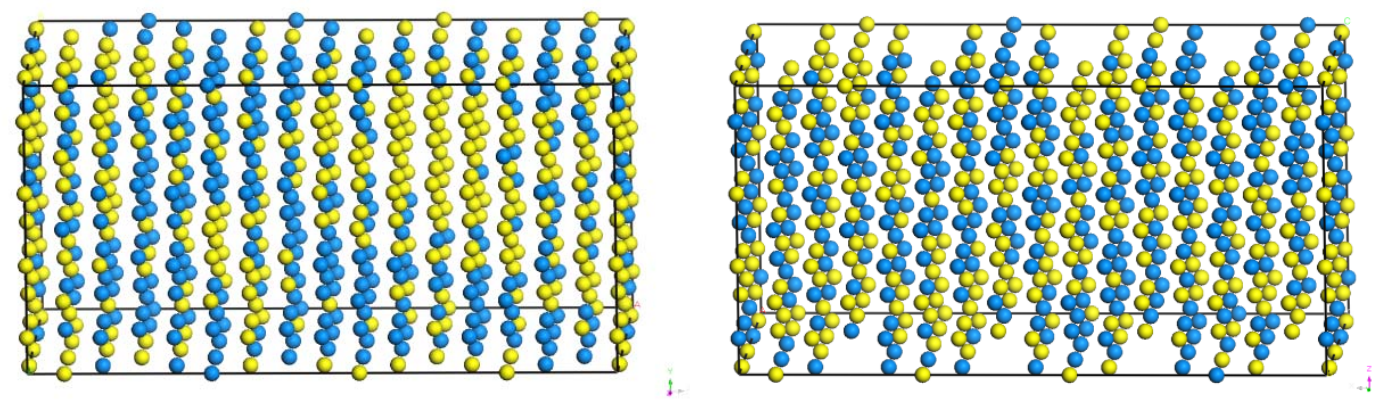

Fig5. Monte Carlo simulation results of the $\mathrm{UO}_{2}-\mathrm{HfO}_{2}$ binary in the cubic framework (Hf mole fraction $=0.5$ ) in a $8 \times 4 \times 4$ supercell of the most energetically favorable configurations at $100 \mathrm{~K}$ (left) and $3000 \mathrm{~K}$ (right). Only uranium (blue balls) and hafnium (yellow balls) are shown in the figure.

\subsection{Enthalpy and free energy of mixing}

The maximum enthalpies of mixing calculated are about $2.0 \mathrm{~kJ} / \mathrm{mol}$ exchangeable cations in cubic framework (Fig2). Previous reported maximum values of enthalpies of mixing calculated by similar method are $2.7 \mathrm{~kJ} / \mathrm{mol}$ exchangeable cations $\left(\mathrm{UO}_{2}-\mathrm{ThO}_{2}\right), 10 \mathrm{~kJ} / \mathrm{mol}$ exchangeable cations $\left(\mathrm{USiO}_{4}-\mathrm{ThSiO}_{4}\right)$ and $30 \mathrm{~kJ} / \mathrm{mol}$ exchangeable cations $\left(\mathrm{ZrSiO}_{4}-\mathrm{ThSiO}_{4}\right)$ [21, 23]. Small mixing enthalpy values are more likely to lead to small or negative values of the Gibbs free energy of mixing due to the $-\mathrm{T} \Delta \mathrm{S}$ term. For cubic-tetragonal series, the energy of the cubic $\mathrm{UO}_{2}$ end member is very close to the supposed tetragonal $\mathrm{UO}_{2}$, which can be explained by the similarity between their cell parameters (Fig 1, a, b). For the other end member $\mathrm{HfO}_{2}$, however, large energy difference is found, which is resulted from the large differences in the cell parameters. For cubic-monoclinic series, as the difference of the structure for the two end members become even larger than the cubic-tetragonal case, energy differences of the two end members is as large as $16 \mathrm{~kJ} / \mathrm{mol}$ exchangeable cations. In general, the energy difference of the 
end member increase as their structures become less similar with each other, which leads to intersection of the free energy of mixing curves at various compositional range.

\subsection{Phase diagram of $\mathrm{UO}_{2}-\mathrm{HfO}_{2}$}

The free energy of mixing curves can be used to derive the phase diagram of the solid solution. The miscibility gap is the region in the phase diagram where two stable phases will form instead of one. On the free energy of mixing curves where the tangent lines connected two local $\Delta \mathrm{G}$ minima for a given temperature, can be used to determine the minimum and maximum amount of $\mathrm{Hf}$ incorporated into $\mathrm{UO}_{2}$. In cubic structure, the $\mathrm{UO}_{2}-\mathrm{HfO}_{2}$ binary forms a complete solid solution. But the $\mathrm{UO}_{2}-\mathrm{HfO}_{2}$ binary has a wide area of miscibility gap on the phase diagram if solid solutions of two different structures are considered. In cubic-tetragonal series, the miscibility gap was centered at $\mathrm{Hf}$ mole fraction of 0.45 , and the gap becomes narrower with increase of temperature (Fig 6, a). In cubic-monoclinic series (Fig 6, b), the miscibility gap is wider than that of cubic-tetragonal series. Because the results only concerned with the free energies of mixing instead of the absolute values of the free energies on their corresponding structures, the stable structures with certain composition at different temperature cannot be determined from current calculations. But the expected situation is a wider miscibility gap of cubic-monoclinic phase at low temperature, a narrow miscibility gap of cubic-tetragonal phases at medium temperature and complete mixing of cubic phase at high temperature. In order to estimate the temperature range for a stable phase in the phase diagram of $\mathrm{UO}_{2}-\mathrm{HfO}_{2}$ binary, the calculated $\mathrm{UO}_{2}-\mathrm{HfO}_{2}$ phase diagram was compared with the experimental $\mathrm{UO}_{2}-\mathrm{ZrO}_{2}$ phase diagram. 

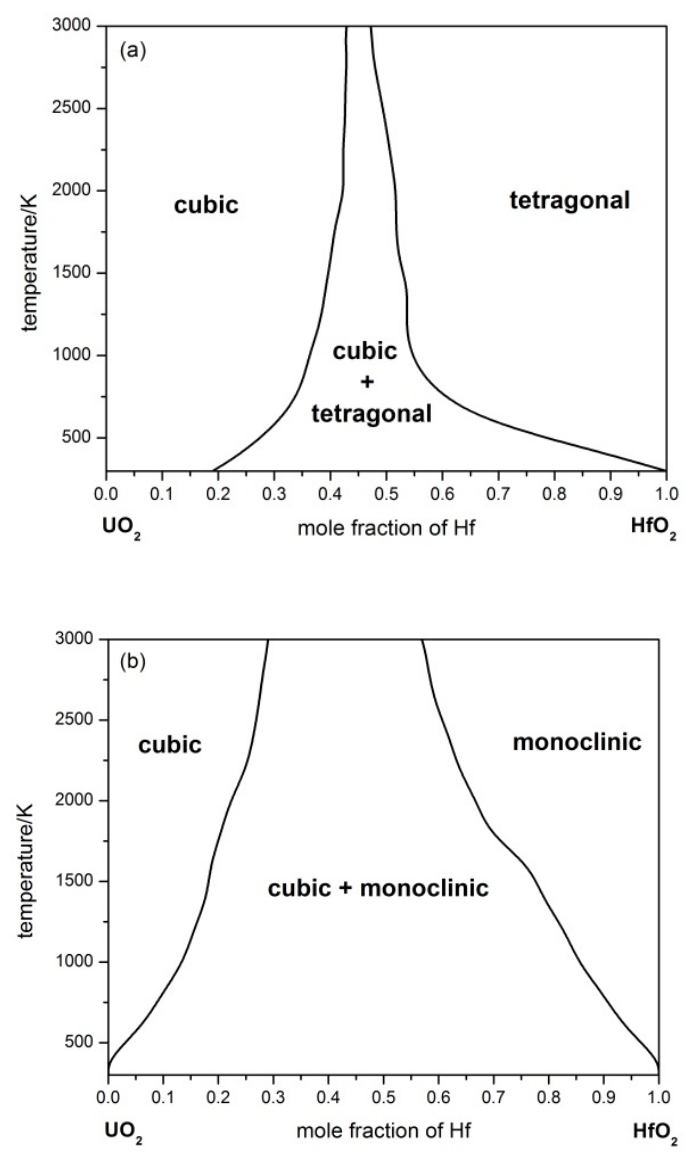

Fig 6. Phase diagrams of $\mathrm{UO}_{2}-\mathrm{HfO}_{2}$ solid solutions with respect to two types of combinations of the end members (cubic-tetragonal (a) and cubic-monoclinic (b)).

\subsection{Comparison to the $\mathrm{UO}_{2}-\mathrm{ZrO}_{2}$ binary solid solution}

Hafnium and zirconium belong to the same group in the periodic table, and the ionic radii and charge of both cations are nearly the same leading to similar chemical behaviors [18, 25]. This can be verified by the occurrence of hafnium in nature where the major source of hafnium is zircon $\left(\mathrm{ZrSiO}_{4}\right)$, in which hafnium take the site of zirconium [26]. High-hafnium zircon with a ratio of $\mathrm{Hf} / \mathrm{Zr}$ about 0.6 has been found in natural zircon minerals [27]. The oxides of hafnium and zirconium, Hafnia and zirconia are considered as similar oxides due to their similar structure and they have been considered to form an ideal solid solution [28]. The $\mathrm{UO}_{2}-\mathrm{ZrO}_{2}$ phase diagram was obtained by quenching samples with a series of chemical compositions and then followed by $\mathrm{XRD}$ to determine the structure. The $\mathrm{UO}_{2}-\mathrm{ZrO}_{2}$ binary forms complete solid solution but the 
details of the phase diagram vary considerably [29]. Within the complete solid solution of $\mathrm{UO}_{2}$ $\mathrm{ZrO}_{2}$, several areas of different phases are presented. The dominant miscibility gap contains cubic and tetragonal phases, and the mixed phase area varies from $\mathrm{Zr}$ mole fraction of $0.4 \sim 0.8$ to as wide as $0.1 \sim 0.9$ at temperature below $2273 \mathrm{~K}$ [30-34]. On the $\mathrm{ZrO}_{2}$ side, small miscibility gap contains monoclinic and tetragonal phases is also presented. Masatomo Yashima [29] compared calculated and experimental phase diagrams of $\mathrm{UO}_{2}-\mathrm{ZrO}_{2}$ binary and obtained the optimized equilibrium phase boundaries, which shows coexistence of both monoclinic and cubic phases at temperature below $1400 \mathrm{~K}$ across the whole composition, immiscible region of tetragonal and cubic phases between 1400 2000K and the complete solid solution of cubic phase with a small miscibility gap close to $\mathrm{ZrO}_{2}$ side at temperature above $2000 \mathrm{~K}$. The ionic radius of $\mathrm{U}^{4+}, \mathrm{Zr}^{4+}$ and $\mathrm{Hf}^{4+}$ are $0.97 \AA, 0.79 \AA$ and $0.78 \AA$, respectively [31]. It is more favorable to substitute small cations with large cations other than introduce large cations into a small lattice. Therefore, the substitution of Hf with $U$ will be energetically more favorable than $\mathrm{Zr}$ substituting $U$ due to its small size, which could result in smaller miscibility gap in $\mathrm{UO}_{2}-\mathrm{HfO}_{2}$ system. Moreover, formation of solid solution decreases the phase transformation temperatures from monoclinic to tetragonal and from tetragonal to cubic. Based on the comparison with $\mathrm{UO}_{2}-\mathrm{ZrO}_{2}$ solid solution, the estimated phase diagrams of $\mathrm{UO}_{2}-\mathrm{HfO}_{2}$ binary are obtained (Fig 7).

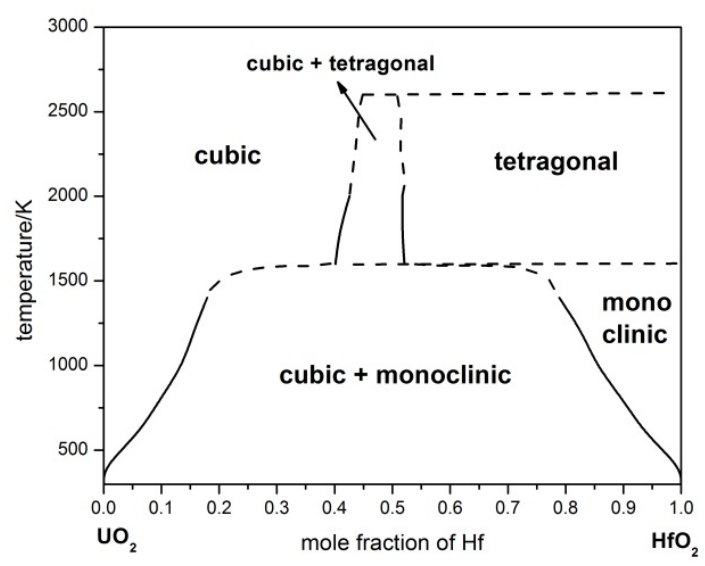

Fig 7. Phase diagram of $\mathrm{UO}_{2}-\mathrm{HfO}_{2}$ solid solution with consideration of phase transition. The dash lines indicate the estimated phase boundary through comparison with the phase diagram of the $\mathrm{UO}_{2}-\mathrm{ZrO}_{2}$ binary solid solution. 
Solid solutions close to the $\mathrm{HfO}_{2}$ side undergo three stages of phase transitions. At temperatures below $1400 \mathrm{~K}$, the wide miscibility gap region of $\mathrm{UO}_{2}-\mathrm{HfO}_{2}$ binary with cubic and monoclinic structures was found (Fig 7), which is narrower than the situation of the $\mathrm{UO}_{2}-\mathrm{ZrO}_{2}$ binary. When the temperature is above $1500 \mathrm{~K}$, a small miscibility gap between $\mathrm{Hf}$ mole fractions of $0.40 \sim 0.52$ exists, followed by the complete solid solution with the cubic structure at temperature above $2600 \mathrm{~K}$. In this strongly non-ideal $\mathrm{UO}_{2}-\mathrm{HfO}_{2}$ binary solid solution, the phase transition regions between monoclinic to cubic and tetragonal to cubic are obscured by the miscibility gap. The reported phase diagram of the $\mathrm{UO}_{2}-\mathrm{ZrO}_{2}$ binary has a wider miscibility gap, reflecting the more covalent bonding of $\langle\mathrm{Zr}-\mathrm{O}\rangle$ as compared with that of $\langle\mathrm{Hf}-\mathrm{O}>$.

\section{Conclusions}

The thermodynamic mixing properties, including enthalpy, free energy and entropy of mixing were calculated in the $\mathrm{UO}_{2}-\mathrm{HfO}_{2}$ binary within cubic, tetragonal and monoclinic frameworks, respectively. The estimated phase diagrams of the $\mathrm{UO}_{2}-\mathrm{HfO}_{2}$ binary with consideration of phase transition were obtained by comparing to the phase diagram of the $\mathrm{UO}_{2}-\mathrm{ZrO}_{2}$. The calculated phase diagram of the $\mathrm{UO}_{2}-\mathrm{HfO}_{2}$ binary indicates the complete mixing of the solid solution with the cubic structure at high temperature. Close to the compositional range of the $\mathrm{UO}_{2}$ end member, uranium-rich solid solutions exsolve as the temperature decrease into the miscibility gap. The calculated phase diagram indicates a tendency to form the monoclinic hafnium-rich phase in the matrix of isometric uranium-rich solid-solution as the fuel cools. Cubic to monoclinic phase transitions of the $\mathrm{UO}_{2}-\mathrm{HfO}_{2}$ binary also involves about $7-10 \%$ increase in the cell volume. The smaller miscibility gap found in the $\mathrm{UO}_{2}-\mathrm{HfO}_{2}$ binary comparing with that of the $\mathrm{UO}_{2}-\mathrm{ZrO}_{2}$ reflects the more covalent bonding of $<\mathrm{Zr}-\mathrm{O}>$ than that of $<\mathrm{Hf}-\mathrm{O}>$.

\section{Acknowledgements}

This work is supported by the US Department of Energy, Nuclear Energy University Programs (NEUP) grant (DE-AC07-05ID14517). 


\section{References}

[1] J.P.A.R. M.L. Grossbeck, Tim Bigelow, Development of improved burnable poisons for commercial nuclear power reactors, University of Tennessee, Knoxville, 2003, 9-12; 69-72.

[2] G.F. C.Collette, F, Abadia, and A. Darraud, Operating experience of the Fragema Gadolinia Bearing Fuel Assembly, in, IAEA, Vienna, 1987, 225.

[3] R. Nijsing, TEMPERATURE AND HEAT FLUX DISTRIBUTION IN NUCLEAR FUEL ELEMENT RODS, Nuclear Engineering and Design, 4 (1966) 11-17.

[4] X.H. Luo, W. Zhou, S.V. Ushakov, A. Navrotsky, A.A. Demkov, Monoclinic to tetragonal transformations in hafnia and zirconia: A combined calorimetric and density functional study, Physical Review B, 80 (2009) 1-11.

[5] R. Terki, G. Bertrand, H. Aourag, C. Coddet, Cubic-to-tetragonal phase transition of HfO2 from computational study, Materials Letters, 62 (2008) 1484-1486.

[6] R. Ruh, H.J. Garrett, R.F. Domagala, N.M. Tallan, SYSTEM ZIRCONIA-HAFNIA, Journal of the American Ceramic Society, 51 (1968) 23-27.

[7] R. Ruh, P.W. Corfield, CRYSTAL STRUCTURE OF MONOCLINIC HAFNIA AND COMPARISON WITH MONOCLINIC ZIRCONIA, Journal of the American Ceramic Society, 53 (1970) 127-129.

[8] G.M. Wolten, DIFFUSIONLESS PHASE TRANSFORMATIONS IN ZIRCONIA AND HAFNIA, Journal of the American Ceramic Society, 46 (1963) 418-422.

[9] M.F. Trubelja, Ionic conductivity in the hafnia-rare earth systems and phase equilibria in the system hafnia-zirconia-yttria: Materials science and engineering, Pennsylvania state university, 1987,1-7.

[10] G.S. Cerefice, Environmental behavior of hafnium: The impact on the disposition of weapons-grade plutonium: Nuclear Engineering, Massachusetts Institute of Technology, Massachusetts, 2001.

[11] V.L. Vinograd, M.H.F. Sluiter, B. Winkler, A. Putnis, U. Halenius, J.D. Gale, U. Becker, Thermodynamics of mixing and ordering in pyrope-grossular solid solution, Mineralogical Magazine, 68 (2004) 101-121.

[12] U. Becker, A. Fernandez-Gonzalez, M. Prieto, R. Harrison, A. Putnis, Direct calculation of thermodynamic properties of the barite/celestite solid solution from molecular principles, Physics and Chemistry of Minerals, 27 (2000) 291-300.

[13] U. Becker, K. Pollok, Molecular simulations of interfacial and thermodynamic mixing properties of grossular-andradite garnets, Physics and Chemistry of Minerals, 29 (2002) 52-64.

[14] M.T.D. A. Bosenick, E.R. Myers, E.J. Palin, C.I. Sainz-Diaz, B.S. Guiton, M.C. Warren, M.S. Craig, S.A.T. Redfern, Computational methods for the study of energies of cation distributions: applications to cationordering phase transitions and solid solutions, Mineralogical Magazine, 65 (2001) 193-219.

[15] M.T. Dove, Computer simulations of solid solutions, EMU notes in mineralogy, 2001,225-230.

[16] M.T.D. M.C. Warren, E.R. Myers, A.Bosenick, E.J. Palin, C.I. Sainz Diaz, B.S. Guiton and S.A.T. Redfern, Monte carlo methods for the study of cation ordering in minerals, Mineralogical Magazine, 65 (2001) 221-248.

[17] R.A.B. John W. Anthony, Kenneth W. Bladh, and Monte C. Nichols, Uraninite: Handbook of mineralogy, Mineralogical Society of America, Chantilly, VA, 2001.

[18] J. Adam, M.D. Rogers, THE CRYSTAL STRUCTURE OF ZRO2 AND HFO2, Acta Crystallographica, 12 (1959) 951-951.

[19] C.E. Curtis, L.M. Doney, J.R. Johnson, SOME PROPERTIES OF HAFNIUM OXIDE, HAFNIUM SILICATE, CALCIUM HAFNATE, AND HAFNIUM CARBIDE, Journal of the American Ceramic Society, 37 (1954) 458465.

[20] W. R.W.G., Crystal structures 1, second ed., Interscience Publishers, New York, 1963. 
[21] E.D.A. Ferriss, R.C. Ewing, U. Becker, Simulation of thermodynamic mixing properties of actinidecontaining zircon solid solutions, American Mineralogist, 95 (2010) 229-241.

[22] M. Reich, U. Becker, First-principles calculations of the thermodynamic mixing properties of arsenic incorporation into pyrite and marcasite, Chemical Geology, 225 (2006) 278-290.

[23] L.C. Shuller, R.C. Ewing, U. Becker, Thermodynamic properties of $\mathrm{Th}(\mathrm{x}) \mathrm{U}(1-\mathrm{x}) \mathrm{O}(2)(0<\mathrm{x}<1)$ based on quantum-mechanical calculations and Monte-Carlo simulations, J. Nucl. Mater., 412 (2011) 13-21.

[24] L.C.E. Shuller-Nickles, R. C. Becker, U, Atomistic calculations of the thermodynamic properties of mixing for tetravalent metal dioxide solid solutions: (Zr, Th, Ce)O2, Journal of Solid State Chemistry, 197 (2013) 550-559.

[25] W.J. Zheng, K.H. Bowen, J. Li, I. Dabkowska, M. Gutowski, Electronic structure differences in $\mathrm{ZrO}(2)$ vs HfO(2), Journal of Physical Chemistry A, 109 (2005) 11521-11525.

[26] N. Korte, M. Kollenbach, S. Donivan, THE DETERMINATION OF URANIUM, THORIUM, YTTRIUM, ZIRCONIUM AND HAFNIUM IN ZIRCON, Analytica Chimica Acta, 146 (1983) 267-270.

[27] A.A. Levinson, R.A. Borup, HIGH HAFNIUM ZIRCON FROM NORWAY, American Mineralogist, 45 (1960) 562-565.

[28] C. Wang, M. Zinkevich, F. Aldinger, The zirconia-hafnia system: DTA measurements and thermodynamic calculations, Journal of the American Ceramic Society, 89 (2006) 3751-3758.

[29] M. Yashima, T. Koura, Y. Du, M. Yoshimura, Thermodynamic assessment of the zirconia-urania system, Journal of the American Ceramic Society, 79 (1996) 521-524.

[30] G.M. Wolten, SOLID PHASE TRANSITIONS IN THE UO2-ZRO2 SYSTEM, Journal of the American Chemical Society, 80 (1958) 4772-4775.

[31] W.A. Lambertson, M.H. Mueller, URANIUM OXIDE PHASE EQUILIBRIUM SYSTEMS .3. UO2-ZRO2, Journal of the American Ceramic Society, 36 (1953) 365-368.

[32] G.M. Wolten, A NOTE ON THE SYSTEM UO2-ZRO2, Journal of the American Ceramic Society, 44 (1961) 148-148.

[33] P.E. Evans, INTERPRETATION OF THE SYSTEM UO2-ZRO2, Journal of the American Ceramic Society, 44 (1961) 631-631.

[34] I. Cohen, B.E. Schaner, A METALLOGRAPHIC AND X-RAY STUDY OF THE UO2-ZRO2 SYSTEM, J. Nucl. Mater., 9 (1963) 18-52. 


\title{
Activation energetics of actinide diffusion in $\mathrm{UO}_{2}$ from first-principles calculations
}

\author{
Jianwei Wang*, Udo Becker \\ Department of Earth and Environmental Sciences, University of Michigan, 1100 N. University Ave., Ann Arbor, MI 48109-1005, USA
}

\section{A R T I C L E I N F O}

\section{Article history:}

Received 27 April 2012

Accepted 10 October 2012

Available online 18 October 2012

\begin{abstract}
A B S T R A C T
Diffusion of actinides in uranium dioxide plays an important role in determining thermodynamic and mechanic properties of the material. Activation energies of Th, $U, N p$, and Pu diffusion in uranium dioxide were systematically studied using first-principles calculations. The generalized gradient approximation and projector-augmented wave methods with on-site Coulomb repulsive interaction were applied within Density Functional Theory and Plane Wave framework. Two diffusion paths, one along the lattice $\langle 110\rangle$ direction and the other along the lattice $\langle 100\rangle$ direction, were examined in the face-centered cubic $\mathrm{UO}_{2}$ structure. The results show that the $\langle 110\rangle$ path has lower migration energy than the $\langle 100\rangle$ path. Under the assumption of a vacancy-assisted jump diffusion mechanism, the major contribution to the activation energy is the migration energy, followed by the vacancy formation energy and vacancy binding energy, where the last has the lowest contribution. However, differences in the activation energies among different actinides stem from both the migration and vacancy binding energies, both of which decrease with atomic number. While discrepancies between the absolute values of the calculated and experimentally observed activation energies remain, this study shows a correlation between activation energy and atomic number and an asymptotic relation between activation energy and ionic radius of the actinides. The present study suggests that the migration of the actinides through the uranium dioxide lattice is closely correlated to the number of $5 \mathrm{f}$ electrons and the size of the diffusing atoms.
\end{abstract}

(c) 2012 Elsevier B.V. All rights reserved.

\section{Introduction}

Used nuclear fuel from commercial reactors is composed of 9599\% uranium dioxide and other actinides. These actinide elements are expected to form solid solutions with $\mathrm{UO}_{2}$ [1-6]. Stabilities of these solid solutions will affect their properties, e.g., chemical and mechanical stabilities, relevant to reprocessing or geologic disposal of the used nuclear fuel. Therefore, it is important to know whether phase separation or phase aggregation of the different oxides of these solid solutions will form and to what extent the ordered phases will form in these solid solutions. Recently, theoretical calculations have been performed to understand thermodynamic stabilities of a range of solid solution series related to the nuclear fuel including $(\mathrm{U}, \mathrm{Th}) \mathrm{O}_{2}$ and $(\mathrm{U}, \mathrm{Np}) \mathrm{O}_{2}$ solid solution series $[1,2]$. Such studies significantly improve the understanding of the thermodynamically stable compositions of these different solid solutions at different temperatures. If a solid solution is thermodynamically favorable, the oxide may form a single phase with mixed actinide elements. If a solid solution is not thermodynamically favorable, the oxide may or may not form separate phases, which is largely controlled by the kinetics of the phase separation or exsolution process. In this process, actinide diffusion in uranium dioxide plays an essential role in determining the kinetics whether

\footnotetext{
* Corresponding author. Tel.: +1 734615 4076; fax: +1 7347634690 .

E-mail address: jwwang@umich.edu (J. Wang).
}

phase separation actually occurs in case the thermodynamics is favorable for such a separation. In addition, diffusion of actinides in $\mathrm{UO}_{2}$ fuel also plays a role in determining the kinetics of the microstructure evolution such as structural deformation and void formation of the fuel at operation conditions in nuclear reactors. Fission gas diffusion and associated gas bubble formation in $\mathrm{UO}_{2}$ have been shown to be largely coupled or controlled by diffusion of $U$ atoms in the structure $[7,8]$. Thus, in order to fully understand phase stability and separation of actinide solid solutions, microstructure revolution, and fission gas diffusion in the fuel, kinetics of the actinide diffusion is necessary and essential.

Experiments have been performed to measure actinide diffusion properties in $\mathrm{UO}_{2}$ [9-14]. However, the measured diffusion properties for the same species vary greatly among different experimental observations. For instance, the experimental activation energy of $\mathrm{U}$ diffusion in $\mathrm{UO}_{2}$ and $\mathrm{UO}_{2+x}$ covers a wide range from $2.3 \mathrm{eV}$ to $4.6 \mathrm{eV}$, reported in a review paper [15]. This variation is largely caused by different stoichiometries of $\mathrm{UO}_{2+x}$ (hyperstoichiometric vs. hypostoichiometric), different samples (single crystal vs. polycrystal) used in those experiments, and different experimental conditions and techniques for the measurements. Because the mechanism of actinide metal diffusions in $\mathrm{UO}_{2}$ may vary at different conditions, it is a great challenge to understand and interpret those experimental observations $[11,14]$. Different mechanisms, such as bulk diffusion, surface diffusion, and grain boundary diffusion have been recognized $[9,16,17]$. However, there is still 
a lack of understanding of the diffusion mechanisms at the atomic scale. In addition, it is unknown how different kinds of defects (e.g., vacancies, interstitial, Schottky defects, and defect clusters) in $\mathrm{UO}_{2}$ would affect the activation energies of actinide diffusions in $\mathrm{UO}_{2}$ and how chemical bonding around these defects would affect the diffusion properties. Theoretical calculations including molecular dynamics simulations and $a b$ initio calculations have advantages not only in calculating fundamental physical properties of $\mathrm{UO}_{2}$ but also in providing atomic-scale mechanisms and insights for the understanding of the diffusion processes. Although it is still a great challenge to accurately describe actinide dioxides using theoretical calculations, DFT methods have been recently applied to understand the basic properties of actinide dioxides including the electronic structure [7,18-27], defects [7,18,20,28-33], thermodynamics [1,34], thermal properties [35-37], and diffusion properties $[7,8,38-40]$ of $\mathrm{UO}_{2}$. The activation energy of oxygen diffusion in $\mathrm{UO}_{2}$ has been calculated by DFT methods [38-40] and molecular dynamics simulations [41]. These studies have provided essential basics to further address the challenges in the understanding of defects and diffusion properties of $\mathrm{UO}_{2}$. However, actinide diffusions in $\mathrm{UO}_{2}$ have been largely underexplored except for some early experimental studies [12,14]. The stoichiometric effect of $\mathrm{UO}_{2+x}$ on the activation energy of $U$ diffusion in uranium dioxide has been formulated thermodynamically and the Arrhenius activation energy varies with $\mathrm{x}: \sim 2.6 \mathrm{eV}$ for $\mathrm{UO}_{2+x}(x \geqslant 0.0), \sim 5.6 \mathrm{eV}$ for $\mathrm{UO}_{2}$, and $\sim 7.8 \mathrm{eV}$ for $\mathrm{UO}_{2-x}(x \leqslant 0.02)[14,42]$. This large variation of the activation energy cannot be explained by a mechanism involving only one $U$ vacancy $[7,8]$. Further investigations reveal that $U$ vacancy diffusion may involve clusters of at least two $U$ vacancies, probably coordinated with additional $O$ vacancies as suggested in a recent article [7]. Thus, the details of atomic-scale mechanisms to explain the experimental activation energies are still underdeveloped.

In this study, first-principles calculations were performed to understand diffusion energetics of actinide impurities in $\mathrm{UO}_{2}$. A vacancy-assisted mechanism was employed for the purpose in this study. This mechanism is also assumed in most theoretical studies in the literature $[7,43,44]$, although in general, both vacancyassisted diffusion and interstitial diffusion mechanisms are possible. The mechanism may involve an impurity atom and a U vacancy or vacancy clusters such as $\mathrm{UO}$ and $\mathrm{UO}_{2}$ [7,43]. For simplicity, a vacancy-assisted mechanism involving one $U$ vacancy and an actinide impurity atom was modeled in this study. The focus is on the trend of the diffusion energetics of different actinides including Th, $\mathrm{U}, \mathrm{Np}$, and Pu. Two different diffusion paths were examined. The variations of the calculated activation energies are rationalized by considering atomic number and ionic radius of the diffusion impurity actinide atoms. Discussions are also made to understand discrepancies between the theoretical calculations and experimental observations on the activation energies and challenges in understanding the diffusion processes in uranium dioxide.

\section{Methods}

Quantum-mechanical calculations were performed using Density Functional Theory (DFT) framework and Plane Wave basis sets as implemented in VASP [45]. The Projector-Augmented Wave method [46] and exchange-correlation as parameterized by Perdew-Wang functional $[47,48]$ were applied in the generalized gradient approximation [49]. The pseudopotentials supplied by VASP were used in the calculations. The $5 \mathrm{f}, 6 \mathrm{~s}, 6 \mathrm{p}, 6 \mathrm{~d}$, and $7 \mathrm{~s}$ electrons in $U$ are treated as valence electrons (total of 14 valence electrons) and the core electrons have [Xe] configuration. For Th, $\mathrm{Np}$, and $\mathrm{Pu}$, the core also has [Xe] configuration with 12,15 , and 16 electrons, respectively, treated as valence electrons. For $\mathrm{O}$, the core has [He] configuration, and the rest $(2 \mathrm{~s}, 2 \mathrm{p})$ are treated as valence electrons (six electrons). The on-site Coulomb interaction with rotationally invariant DFT $+U$ (italicized $U$ stands for Hubbard $U$, plain $U$ for uranium) method introduced by Liechtenstein et al. [50] and a fully relativistic calculation for the core-electrons and scalar relativistic approximation for the valence electrons were employed to approximate relativistic effects and electron correlation effects for the $5 \mathrm{f}$ electrons. The on-site Coulomb interaction parameters are $U=3.8$ and $J=0.4$, which were tuned to reproduce the experimentally observed band gap width of $\mathrm{UO}_{2}$ [51]. Similar methods have been used in a previous study and reasonably reproduced a number of experimentally observed properties of uranium dioxide, including the crystal structure, electronic structure, and phase transition pressure [52].

The computations were performed based on a $2 \times 2 \times 2$ supercell of $\mathrm{UO}_{2}$. Fig. 1 shows the crystal structure of $\mathrm{UO}_{2}$. Energy cutoff for the plane-wave basis set was $520.00 \mathrm{eV}$ (or $38.2 \mathrm{Ry}$ ) for all calculations. The Monkhorst-Pack scheme for integration in the Brillouin zone was adopted, and a $2 \times 2 \times 2 k$-point grid was used. Using a $3 \times 3 \times 3 k$-point grid did not improve the convergence. A $1 k$ antiferromagnetic order was used with alternating spins on $U$ parallel to the $\langle 100\rangle$ directions. Although a recent experimental result supports a $3 k$ magnetic order with spins on $U$ parallel to $\langle 111\rangle$ [53], the $1 k$ spin order still is a good approximation of the $3 k$ order with a difference in total energy of $\sim 2.4 \mathrm{meV} / 12$ atoms cell (i.e., $0.2 \mathrm{meV} /$ atom) as demonstrated in the previous study [28]. Thus, the results based on the $1 k$ order are not expected to strongly affect the conclusions. Collinear magnetic calculations were performed. Spin-orbit coupling was not included in the calculations. All the calculations were performed without allowing a change of the cell shape but the volumes were pre-relaxed to their equilibrium volumes, which constrains the computational cell to be cubic while allowing local structure changes around defects. Fixing the computational cell shape only causes a small error in the calculated energy with respect to relaxing the cell shape as demonstrated in a previous study [52].

Electronic metastable states of cubic fluorite $\mathrm{UO}_{2}$ have been documented and methods to achieve low energy states have been proposed in the literature [21,54-56]. In a method of enumerating the initial occupation matrix of the $U 5 f$ electrons, it was suggested that monitoring the $U 5 \mathrm{f}$ occupation matrices and allowing the $5 \mathrm{f}$ electrons to break the cubic symmetry are necessary [21]. In a

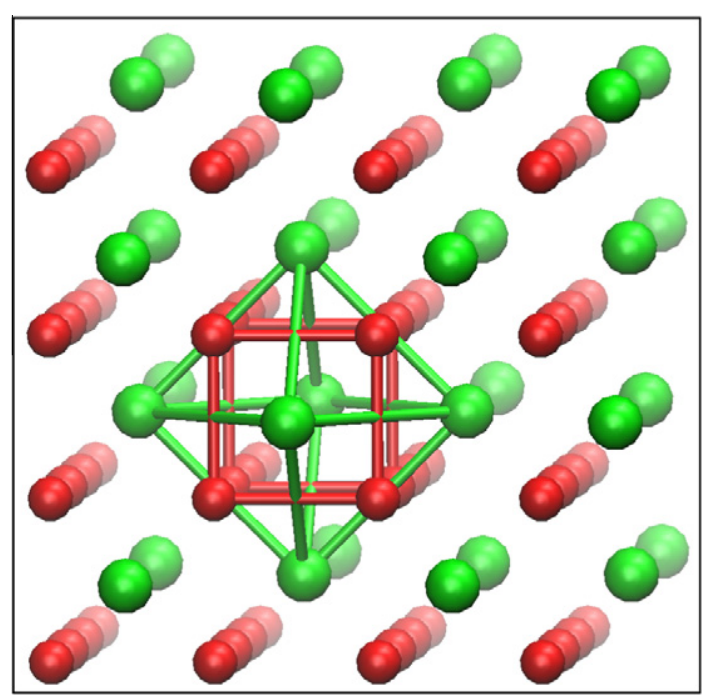

Fig. 1. Crystal structure of $\mathrm{UO}_{2}$ in 96 atoms cell. The $\mathrm{U}$ octahedron and $\mathrm{O}$ cube surrounding the lattice interstitial site are highlighted by connecting the neighboring $\mathrm{U}$ and $\mathrm{O}$ atoms. 
Hubbard $U$-ramping scheme, the $U$ parameters are gradually ramped up from zero to a desired value in a series of calculations [55]. In this study, the initial occupation matrix for $U$ atoms was not controlled but the occupation matrix was checked for metastability by following the change of the occupation matrices during the convergence, and by comparing the converged occupation matrices with the published ground state occupation matrices in the literature [28] and with the converged occupation matrices using one of literature proposed methods for obtaining the ground state of $\mathrm{UO}_{2}$ such as the U-ramping method [55]. In order for the electronic structure being allowed to break symmetry, the supercell symmetry was switched off in all calculations. As demonstrated in previous calculations [21,28,52], switching off the symmetry and using the rotationally invariant Liechtenstein scheme [50] help the systems reaching their ground states. Even if the electronic ground states were not reached, the converged states are expected to have a small energy difference from the true ground states. The average of this energy difference, proposed in this study as the error of the calculations without using the ramping scheme, was estimated by comparing the energy of the asconverged states with the results using the Hubbard $U$-ramping method for a number of different structural configurations. The error from such calculations is $\sim 0.2 \mathrm{eV} /(96$ atom cell) (i.e., $\sim 2 \mathrm{meV} /$ atom).

\section{Results and discussion}

\section{1. $D F T+U$ description of $U O_{2+x}$}

In order to reasonably predict diffusion properties of actinides in $\mathrm{UO}_{2+\chi}$, the crystal structure, electronic structure, and defect properties of $\mathrm{UO}_{2}$ have to be calculated as accurately as possible. In addition, the phase behavior of $\mathrm{UO}_{2}$ under pressure also needs to be predicted reasonably well. At high pressures, inter-atomic bond distances are shorter than at low pressures. A better prediction on the energetics at high pressures provides confidence on the calculated energetics of the diffusion, especially energies where the diffusing atom migrating away from its equilibrium position with shorter bond distances.

The basic crystal structure and electronic structure of $\mathrm{UO}_{2}$ were well reproduced using the methods described in the previous section. The calculated band gap is $2.1 \mathrm{eV}$, consistent with the experimental value $2.1 \mathrm{eV}$ [51]. The calculation suggests that the $\mathrm{U}$ formal oxidation state is +4 with two unpaired $5 \mathrm{f}$ electrons. The calculated density of states shows that at the top of the valence band is mainly $\mathrm{U}-5 \mathrm{f}$ band, and the $0-2 \mathrm{p}$ band is $\sim 3-6 \mathrm{eV}$ below the Fermi level, consistent with the result from photoemission spectroscopic experiments [57]. The calculated unit cell constant is $5.54 \AA$ with the GGA+U $(U=3.8, J=0.4)$ methods, $\sim 1 \%$ higher than $5.47 \AA$ of an experimental value at ambient temperature [58]. Similar results have been obtained in a previous study using similar methods [20]. The bulk modulus, 193.4 GPa, calculated by fitting a dataset of the total energy as a function of volume using the Birch-Murnaghan equation of state [59], is in agreement with 207 GPa from a previous experiment [60] and 195.4 GPa from a recent DFT calculation [20]. At high pressure, $\mathrm{UO}_{2}$ with a cubic fluorite structure is transformed to a high-pressure phase with an orthorhombic cotunnite structure. The phase transition pressure was estimated by the slope of the cotangent of the two-phase curves from a plot of the calculated total energy as a function of volume. For stoichiometric $\mathrm{UO}_{2}$, the calculated transition pressure is $17 \mathrm{GPa}$, much below the experimentally observed transition pressures of $29 \mathrm{GPa}$ [61] and $42 \mathrm{GPa}$ [60]. This discrepancy leads to question if $\mathrm{DFT}+U$ can be used to accurately describe $\mathrm{UO}_{2}$ at high pressures $[20,22]$. For $\mathrm{UO}_{2 \cdot 03}$, our calculations show that the calcu- lated transition pressure is $27 \mathrm{GPa}$. This result suggests that the phase transition pressure is very sensitive to a small amount of excess oxygen in hyperstoichiometric $\mathrm{UO}_{2+x}$. The details of these calculations are reported in a separate paper and are summarized in this contribution for completeness [52].

\subsection{Activation process of actinide impurity diffusion in $\mathrm{UO}_{2}$}

Under the assumption of a vacancy-assisted diffusing mechanism, for an impurity diffusing atom (i.e., Th, $\mathrm{U}, \mathrm{Np}$, and $\mathrm{Pu}$ ) to $\mathrm{mi}-$ grate, a vacancy has to be formed, and the vacancy has to be neighboring the diffusing atom, driven by an association energy or binding energy between the vacancy and the diffusing atom. Populations of $U$ vacancies and those binding to the diffusing atoms obey statistical mechanics principles and the Boltzmann distribution, which are thermally activated. Once there is a vacancy neighboring the diffusing atom, the diffusing atom can migrate from its lattice site to the vacancy site. As illustrated in Fig. 2, there are three components to the activation energy, which is defined as [62]:

$\Delta H_{a}=\Delta H_{v}-\Delta H_{v b}+\Delta H_{m}$

where $\Delta H_{v}$ is the vacancy formation energy, $\Delta H_{v b}$ is the vacancy binding energy, and $\Delta H_{m}$ is the migration energy.

For the vacancy formation energy calculation, one $U$ atom is deleted from its lattice site, resulting in 95 atoms in the computational supercell. Recent studies show that charged defects in $\mathrm{UO}_{2}$ could be more stable than their neutral ones $[7,18]$. However, charged-state calculations need corrections by artificially assuming a homogeneous background charge [63] and may also cause complications in correctly calculating migration energies. For simplicity, all calculations in this study were done with a charge neutral system, which results in the stoichiometry of the system slightly off $\mathrm{UO}_{2}$. Note that such a configuration is a simplified model to simulate the impurity diffusion. For more accurate models, other types of defects involving one or more $U$ and $O$ vacancies such as Frenkel defects, Schottky defects, and/or defect clusters have to be considered. However, implementing these defect types (i.e., involving more than one site) would make multiple models unavoidable in order to account for the effect of different

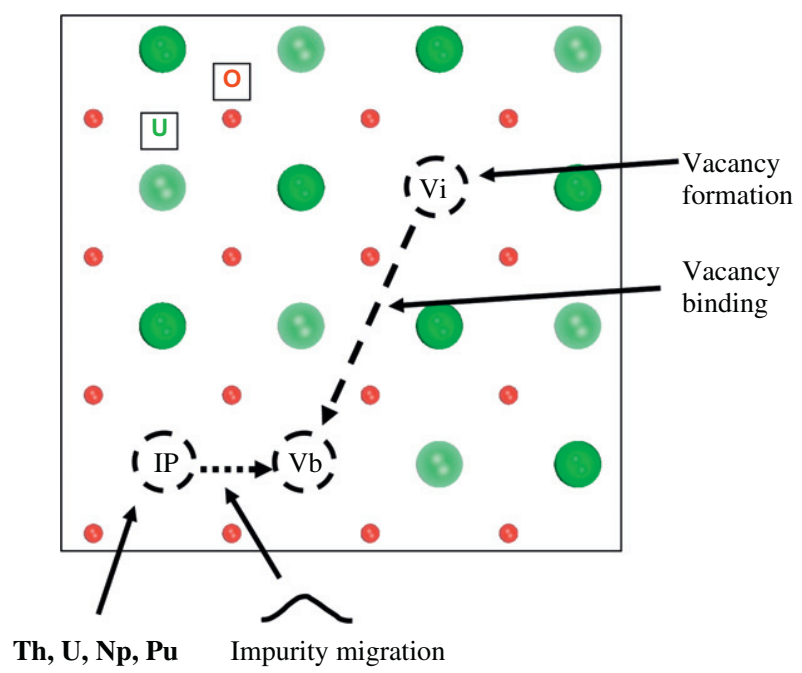

Fig. 2. A schematic view of a interstitial-assisted actinide diffusion mechanism in $\mathrm{UO}_{2}$ lattice projected on (001) plane. Only two layers of $U$ atoms and one layer of $\mathrm{O}$ atoms are shown. Larger balls are $U$ atoms. The small balls are oxygen atoms. Different cueing balls for $\mathrm{U}$ atoms indicate the atoms below and above the oxygen atom layer. Dashed circles are the locations of vacancies or actinide atoms. Dashed arrows show moving direction of the vacancy or diffusion actinide atom. 
configurations of the defect sites in the diffusion calculations. Thus, for a qualitative understanding of the trend of diffusion energetics of an actinide impurity in uranium dioxide as a function of atomic number, in this study, the simplified model with a neutral charge was employed.

In the dilute limit where the concentration of an impurity actinide is small, the effect of the impurity atom on the U vacancy formation is ignored because, on average, the impurity is more than a few bonds away from the vacant site. The vacancy formation energy is calculated based on the following equation:

$29 / 32 \mathrm{U}_{32} \mathrm{O}_{64}+2 \delta-\mathrm{UO}_{3}=\mathrm{U}_{31} \mathrm{O}_{64}$

where $\delta-\mathrm{UO}_{3}$ was used as a reference phase. Using $\delta-\mathrm{UO}_{3}[64]$ as a reference phase, the calculated vacancy formation energy is $2.1 \mathrm{eV}$ (Table 1). It needs to be mentioned that for a charge-neutral defect, defect formation energies depend on the reference phase or chemical potentials of $U$ and $O$ [65]. If $\alpha$-U was used as a reference phase, the formation energy would be $4.9 \mathrm{eV}$, which is consistent with the value $(4.48 \mathrm{eV})$ in the literature using similar methods and the same reference phase [20]. At oxygen-rich conditions, however, the formation energy of a U vacancy was calculated to be $-6.50 \mathrm{eV}$ [20]. The vacancy formation energy also depends on the stoichiometry of $\mathrm{UO}_{2+\chi}$, the charge state, how symmetry is controlled, if the calculation is done in one computational supercell or two separate supercells, and the size of the supercell being used (i.e., dilution) as demonstrated in a recent study [7]. The calculated values in that study vary widely from $+11.9 \mathrm{eV}$ to $-1.1 \mathrm{eV}$. For the purpose of this study, the defect formation energy of $2.1 \mathrm{eV}$ is used here to understand the trend across the actinides for consistency and simplicity.

Since $\mathrm{O}$ atoms are expected to maintain their oxidation states in $\mathrm{U}_{31} \mathrm{O}_{64}$, removing a $\mathrm{U}$ atom from the system (e.g., $\mathrm{U}_{32} \mathrm{O}_{64}$ ) will result in some of the $U$ atoms donating more electrons to the oxygen atoms and increasing their oxidation states. This can be checked by monitoring the magnetic moments of the $U$ atoms. The calculated results show that there are four $U$ atoms with a magnetic moment of $\sim 1.0 \mu_{B}$ and the rest with a magnetic moment of $\sim 2.0 \mu_{B}$. This partial loss of the magnetic moment can be interpreted as an oxidation state change of the $U$ atoms from $\mathrm{U}^{4+}$ to $\mathrm{U}^{5+}$. It is interesting to observe that none of the $U$ atoms is oxidized from $4+$ to $6+$. The oxidized $U$ atoms are not necessary to be the nearest neighbors of the defect (i.e., the $U$ vacancy). Similar results have been reported in previous studies where $U$ oxidations from $U^{4+}$ to $U^{5+}$ state is induced by interstitial oxygen $[38,66]$. It is expected that structures with four $\mathrm{U}^{5+}$ atoms at different locations with respect to the $U$ vacancy site have different vacancy formation energies. In the present study, a series of calculations were carried out and each has a $U$ vacancy initially located at a different location. The optimizations resulted in the $\mathrm{U}^{5+}$ atoms being located at different locations with respect to the vacancy and the total energy differences are in a range between $0.1 \mathrm{eV}$ and $0.5 \mathrm{eV}$. It is speculated that the energy differences may be caused by all or any combinations of the differences in electrostatic interactions of the defect sites (i.e., the vacancy site and $\mathrm{U}^{5+}$ sites), spin-spin interactions, and chemical bonding of the $\mathrm{U}^{5+}-\mathrm{O}$ bonds. In this study, for a qualitative understanding, the lowest-energy structure of all the possible $\mathrm{U}^{5+}$ config- urations was not sought, which would be a subject of future studies. Instead, an error of $0.3 \mathrm{eV}$ was estimated to the energy of the as-converged structure. In one of the as-converged structures for $\mathrm{U}_{31} \mathrm{O}_{64}, \mathrm{U}^{5+}$ atoms are located two to three bonds away from the vacancy site. Considering together with the error associated with electronic metastability of $0.2 \mathrm{eV} / 96$ atom cell, the error of the calculated vacancy formation energy is $\sim 0.5 \mathrm{eV}$, resulted from the errors related to electronic metastability and $\mathrm{U}^{5+}$ configurations. The error of vacancy binding energies is expected to be at the same level. The error of the calculated migration energies is expected to be on the order of $0.2 \mathrm{eV}$, based on the error associated with the electronic metastability. Thus, the error of the calculated activation energies based on Eq. (1) is $\sim 0.7 \mathrm{eV}$, propagated from its components.

For vacancy binding energy calculations, one $U$ atom was substituted by an impurity atom $\mathrm{M}(\mathrm{M}=\mathrm{Th}, \mathrm{Np}$, and $\mathrm{Pu})$, resulting in a system with a formula of $\mathrm{M}_{1} \mathrm{U}_{30} \mathrm{O}_{64}$. As Fig. 2 illustrates, the binding energy is calculated as the energy difference between a system with a vacancy not bound to the impurity (IP) (Vi, three bonds away from the diffusion impurity atom) and that bound to the impurity ( $\mathrm{Vb}$, a vacancy adjacent to the impurity). A positive value indicates an impurity attractive interaction to the vacancy. The calculated binding energy for each impurity actinide is listed in Table 1. As shown, the binding energy is $-0.3 \mathrm{eV}$ for $\mathrm{Th}, 0.0$ for $\mathrm{U}, 0.3 \mathrm{eV}$ for $\mathrm{Np}$, and $0.4 \mathrm{eV}$ for Pu. Such a trend is counterintuitive because larger impurity atoms (e.g., Th) are expected to be energetically favorable if adjacent to the vacancy than smaller atoms (e.g., Pu). Thus, the binding energy may be largely caused by electronic interactions involving the $5 f$ electrons. For Th at $4+$ charge state without $5 \mathrm{f}$ electrons, the binding energy is negative, and for $\mathrm{Np}$ and $\mathrm{Pu}$, the binding energies are positive, suggesting an increased attractive interaction of the impurities with vacancy as the number of $5 \mathrm{f}$ electrons increases. However, the binding energy contribution to the activation energy is relatively small for the actinides, suggesting that the interactions of the impurities with the vacancies are weak. The calculated magnetic moments of impurity atoms are $\sim 0.0 \mu_{B}$ for Th, $\sim 2.0 \mu_{B}$ for $\mathrm{U}, \sim 3.1 \mu_{B}$ for $\mathrm{Np}$, and $\sim 4.2 \mu_{B}$ for $\mathrm{Pu}$, suggesting that the oxidation states of the impurity atoms (i.e., Th, $\mathrm{Np}$, and $\mathrm{Pu}$ ) do not change when a vacancy is at the neighboring location.

\subsection{Two diffusion paths}

In the face-centered cubic lattice, there are two possible paths for a $U$ atom to migrate to its next neighboring sites. As shown in Fig. 3, one way for the atom to migrate is to jump through the interstitial vacancy site in the lattice. This path is possible because the vacancy may provide some space needed for a diffusing atom to move from one site to the other. The moving direction is along the edge of the cubic lattice or $\langle 001\rangle$ directions, parallel to the principle axes of the crystal structure. The path distance (P-iv) is $5.47 \AA$, which is not the shortest. The shortest-distance path is to move to its nearest $U$ neighboring sites, which is $3.87 \AA$ (P-s). This path is along the diagonal direction of the two principle axes (Figs. 2 and 3). Calculations were performed for both of these two paths for U diffusion.

Table 1

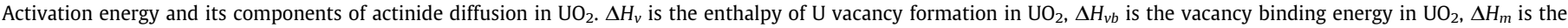
migration energy of actinide in $\mathrm{UO}_{2}$, and $\Delta H_{a}$ is the activation energy of actinide in $\mathrm{UO}_{2}$.

\begin{tabular}{|c|c|c|c|c|c|}
\hline Systems/energy (eV) & $\Delta H_{v}( \pm 0.5)$ & $\Delta H_{v b}( \pm 0.5)$ & $\Delta H_{m}( \pm 0.2)$ & $\Delta H_{a}( \pm 0.7)$ & Experiment $^{\mathrm{a}}$ \\
\hline Th in $\mathrm{UO}_{2}$ & 2.1 & -0.2 & 5.4 & 7.7 & $6.5\left(\mathrm{Th}\right.$ in $\left.\mathrm{ThO}_{2}\right)$ \\
\hline $\mathrm{U}$ in $\mathrm{UO}_{2}$ & 2.1 & 0.0 & 5.2 & 7.3 & $\sim 2.6$ to $\sim 7.8$ \\
\hline $\mathrm{Np}$ in $\mathrm{UO}_{2}$ & 2.1 & 0.3 & 4.9 & 6.7 & \\
\hline $\mathrm{Pu}$ in $\mathrm{UO}_{2}$ & 2.1 & 0.4 & 4.0 & 5.7 & \\
\hline
\end{tabular}

a Matzke [42]. 


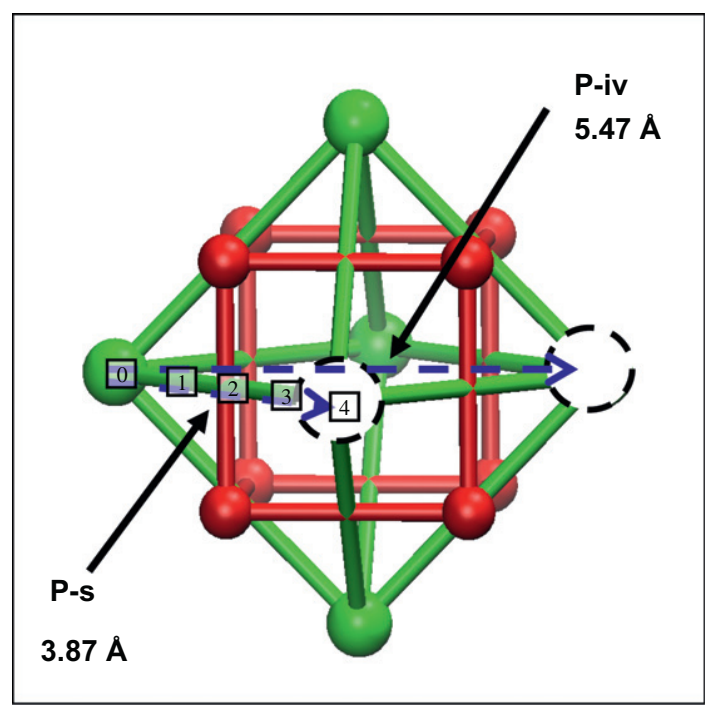

Fig. 3. A representation of two diffusion paths. Only atoms associated with the $U$ octahedron and $O$ cube surrounding the lattice interstitial site are shown. The highlighted atoms and connections with the dashed arrows indicate two diffusion paths, P-iv and P-s. The dashed open circles are the vacant site neighboring the diffusing atom. The numbers indicate the locations of the diffusion impurity for the corresponding NEB images based P-s diffusion path.

There are two methods in the literature to estimate the migration energy of an impurity atom diffusion using static DFT calculations. The Nudged Elastic Band (NEB) method has been widely documented for diffusion calculations [67] and recently has been employed for $\mathrm{O}$ and $\mathrm{U}$ diffusions in $\mathrm{UO}_{2}[7,38,39]$. The other way to calculate the migration energy is step by step moving a diffusion atom from one lattice site to the next along a predefined diffusion path. At each step, the system is optimized (including the coordinates of the diffusion ions perpendicular to the path). In both cases, the energy surface as a function of the diffusion path is generated. The migration energy is estimated as the difference between the maximum and the base of the energy surface. The latter method needs to constrain some atoms in the system, which may cause errors in the calculated results. The climbing image NEB method $(\mathrm{CI}-$ NEB) implemented in VASP was used in the present study [67].

Fig. 4 shows calculated energy surfaces of $\mathrm{U}$ diffusion in $\mathrm{UO}_{2}$ lattice along two diffusion paths. The shortest distance path has actually a lower migration energy $(5.2 \mathrm{eV})$, although, along the diffusion path, there are two oxygen atoms at a very short distance. Counterintuitively, the interstitial vacancy path has a higher migration energy $(8.7 \mathrm{eV})$. Structural analysis shows that along the interstitial path, there are four $U$ atoms at a relatively short distance. The strong repulsion from these $U$ atoms induces a much higher barrier for the $U$ to migrate than that caused by the closeby $\mathrm{O}$ atoms in the case of the shortest diffusion path. Based on this observation, all the rest of the diffusion calculations were carried out only for the shortest distance path. Since the CI-NEB method was used in the calculations, the saddle point is expected to be calculated quite accurately and so is the calculated migration energy. Since $U$-ramping scheme is not implemented in the CI-NEB method with VASP, an error $(0.2 \mathrm{eV})$ is estimated for the migration energy calculations as described in the previous section.

\subsection{Activation energy of actinide diffusion and correlation with atomic number}

The calculated activation energies and their components of different actinides in $\mathrm{UO}_{2}$ are listed in Table 1 . The migration energies
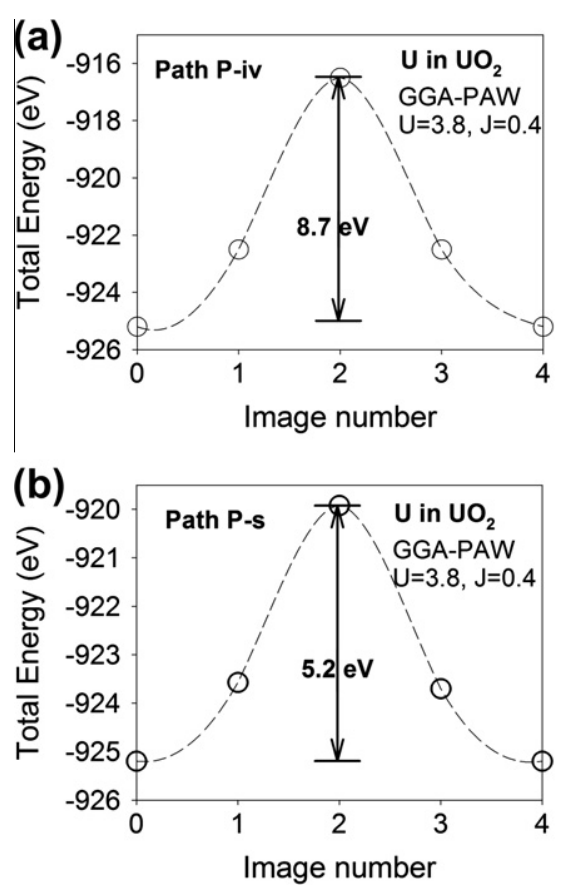

Fig. 4. The energy surface for $U$ diffusion in $\mathrm{UO}_{2}$ along $\langle 100\rangle$ direction through interstitial site, the P-iv path (a), and along $\langle 110\rangle$ direction, the shortest distance, the P-s path (b). The dashed lines are eye-guide. For both of the calculations, five images were used. The horizontal axis is the image numbers in the CI-NEB calculations.

for Th, $\mathrm{U}, \mathrm{Np}$, and $\mathrm{Pu}$ are 5.4, 5.2, 4.9, and $4.0 \mathrm{eV}$, respectively. The migration energy for $\mathrm{U}(5.2 \mathrm{eV})$ is comparable with a previously calculated migration energy of $4.81 \mathrm{eV}$ of $\mathrm{U}$ migrating along the same path and using similar methods [7], but relatively larger than another calculation $(3.09 \mathrm{eV})$, where in the latter case the calculations were performed without the Hubbard $U$ onsite interaction for the $5 \mathrm{f}$ electrons [43]. The calculated migration energy for $\mathrm{Th}$ in $\mathrm{UO}_{2}$ $(5.4 \mathrm{eV})$ is larger than a previous calculation of $\mathrm{Th}$ in $\mathrm{ThO}_{2}(4.47 \mathrm{eV})$ [8]. As shown in Table 1, for different actinide atoms, the migration barrier becomes smaller as atomic number of the actinide atom increases, so is the activation energy from $\mathrm{Th}, \mathrm{U}, \mathrm{Np}$, to $\mathrm{Pu}$. It needs to be mentioned that earlier experimental studies show that the diffusion rates of $\mathrm{Th}, \mathrm{U}$, and $\mathrm{Pu}$ in $\mathrm{ThO}_{2}$ are practically identical [12]. However, in order to compare the calculations with the experimental diffusion rates, attempt frequencies of the actinide diffusions need to be calculated as well, which is a subject of future studies. As shown in Table 1, the major contribution to the activation energy is the migration energy since the binding energy only contributes little to the activation energy for each of the actinides. Nevertheless, the binding energy contributes as much as the migration energy to the difference of the activation energies for most of the actinides. For Th diffusion in $\mathrm{UO}_{2}$, the calculated activation energy, $7.7 \mathrm{eV}$, is higher than the experimental value of $\sim 6.5 \mathrm{eV}$ of $\mathrm{Th}$ in $\mathrm{ThO}_{2}$ [42]. For $\mathrm{U}$ diffusion in $\mathrm{UO}_{2}$, the calculated value of $7.3 \mathrm{eV}$ is near the high end of the experimental values (i.e., 2.6-7.8 eV) using non-irradiated samples of single crystal uranium dioxide, where a tracer was used in the experiment and alpha-energy degradation method was used to measure the tracer penetration $[16,42]$. Although agreement with experiment results is not expected, such discrepancies are a concern for both experiment and calculation. Theoretically, errors may be caused by inaccurate assumptions of the diffusion model and complications from real materials that are difficult to take into consideration in the calculations. For instance, defects near the diffusion path, especially oxygen interstitials, which are common in uranium dioxide, could 
significantly affect all the components of the activation energy as demonstrated in recent theoretical studies [7,8]. Experimentally, bulk diffusion, surface diffusion, and grain boundary diffusion need to be distinguished clearly since each of them should have distinctly different diffusion mechanisms and activation energies. Not all the diffusion experiments are under strictly environmental controls to guarantee the stoichiometry of $\mathrm{UO}_{2}$ during the entire period of the measurements. Such a control is a challenge because $\mathrm{UO}_{2}$ tends to deviate from its stoichiometry at high temperatures, at which most diffusion experiments were conducted. Further theoretical investigations may provide important clues on those discrepancies and valuable insights by considering additional diffusion models and effects of other structural defects and a deviation from $\mathrm{UO}_{2}$ stoichiometry. Even if the above-mentioned problems can be resolved, a comparison of calculated activation energies, especially those based on static energy calculations such as presented here, with experimentally observed Arrhenius activation energies is not straightforward. For a direct comparison between theory and experiment, entropic contributions need to be included in the calculations, finite temperature effects on migration energy calculations should be taken into account, and the entire energy surface along the diffusion path needs to be considered, to name a few.

Fig. 5 shows the calculated activation energy as a function of atomic number, along with ionic radius and metallic radius of the actinides. The activation energy decreases with atomic number and increases as a function of ionic radius (Fig. 6). For actinide metals, localization and delocalization of the $5 \mathrm{f}$ electrons have a strong effect on the sizes and other properties. As the electrons become more delocalized as the series approaches $\mathrm{Pu}$, the size starts to increase. This is not the case for the actinide dioxides. The ionic radius decreases roughly linearly with atomic number as more $5 \mathrm{f}$

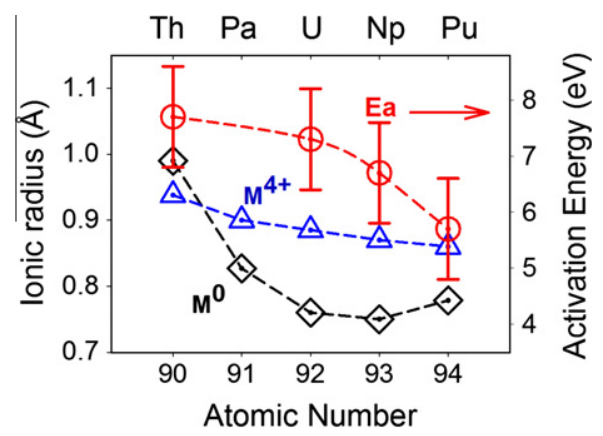

Fig. 5. Activation energy of different actinide atoms in $\mathrm{UO}_{2}$ and ionic radius as a function of atomic number. M0 is for zero valence metal actinide [68], $\mathrm{M}^{4+}$ is for tetravalent actinide cation [68], and Ea stands for the activation energy of the diffusion. The dashed lines are eye-guide and the error bars are the estimated errors.

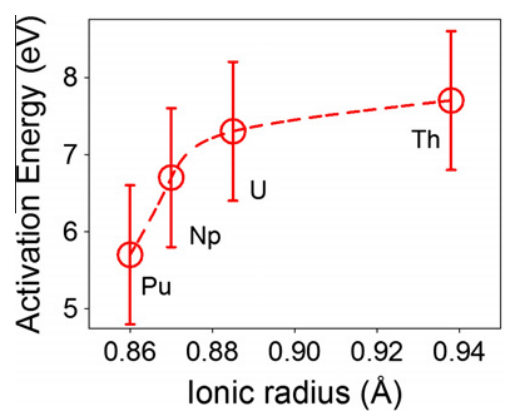

Fig. 6. Activation energy of different actinide atoms in $\mathrm{UO}_{2}$ as a function of ionic radius. The dashed lines are eye-guide and the error bars are the estimated errors. electrons participate in the bonding [68]. Although the absolute values of the calculated activation energies are somewhat higher than the experimental activation energies, the present study shows that the activation energies of actinide diffusions in $\mathrm{UO}_{2}$ have a strong correlation with the atomic number and ionic radius, and such a correlation can be validated by well controlled systematic experimental studies.

\section{Acknowledgements}

This work is supported by the Office of Nuclear Energy, University Program (NEUP) of the U.S. Department of Energy under contract DE-AC07-05ID14517. Computational resources for this project are made available in part by the National Science Foundation through TeraGrid/XSEDE resources from NCSA and NICS under Grants TG-DMR080047 N and TG-DMR100034. This research used resources of the National Energy Research Scientific Computing Center (NERSC), which is supported by the Office of Science of the U.S. Department of Energy under Contract No. DE-AC02$05 \mathrm{CH} 11231$.

\section{References}

[1] L.C. Shuller, R.C. Ewing, U. Becker, Journal of Nuclear Materials 412 (2011) 1321.

[2] L.C. Shuller, N. Pavenayotin, R.C. Ewing, U. Becker, Thermodynamic properties of actinide-oxide solid-solutions, in: Materials for Future Fusion and Fission Technologies, 2009, pp. 95-100.

[3] P. Martin, S. Grandjean, C. Valot, G. Carlot, M. Ripert, P. Blanc, C. Hennig, The Journal of Alloys and Compounds 444 (2007) 410-414.

[4] S. Miwa, M. Osaka, H. Yoshimochi, K. Tanaka, K. Kurosaki, M. Uno, S. Yamanaka, The Journal of Alloys and Compounds 444 (2007) 610-613.

[5] B. Arab-Chapelet, S. Grandjean, G. Nowogrocki, F. Abraham, The Journal of Alloys and Compounds 444 (2007) 387-390.

[6] M. Hirota, K. Kurosaki, D. Setoyama, M. Kato, M. Osaka, T. Narnekawa, M. Uno, S. Yamanaka, Journal of Nuclear Materials 344 (2005) 84-88.

[7] D.A. Andersson, B.P. Uberuaga, P.V. Nerikar, C. Unal, C.R. Stanek, Physical Review B 84 (2011) 054105.

[8] Y. Yun, P.M. Oppeneer, H. Kim, K. Park, Acta Materialia 57 (2009) 1655-1659.

[9] A.C.S. Sabioni, W.B. Ferraz, F. Millot, Journal of Nuclear Materials 278 (2000) 364-369.

[10] A.C.S. Sabioni, W.B. Ferraz, F. Millot, Journal of Nuclear Materials 257 (1998) 180-184.

[11] H. Matzke, Diffusion Processes in Nuclear Fuels, North Holland, Amsterdam, 1992.

[12] H. Matzke, Journal of the Chemical Society, Faraday Transactions 2: Molecular and Chemical Physics 83 (1987) 1121-1142.

[13] H.J. Matzke, Radiation Effects 53 (1980) 219-242.

[14] H. Matzke, Journal of Nuclear Materials 30 (1969) 26-35.

[15] J. Belle, Journal of Nuclear Materials 30 (1969) 3-15.

[16] H. Matzke, Journal of Nuclear Materials 114 (1983) 121-135.

[17] S. Yajima, H. Furuya, T. Hirai, Journal of Nuclear Materials 20 (1966) 162-170.

[18] J.-P. Crocombette, D. Torumba, A. Chartier, Physical Review B 83 (2011) 184107.

[19] R. Devanathan, L. Van Brutzel, A. Chartier, C. Gueneau, A.E. Mattsson, V. Tikare, T. Bartel, T. Besmann, M. Stan, P. Van Uffelen, Energy \& Environmental Science 3 (2010) 1406-1426.

[20] J.G. Yu, R. Devanathan, W.J. Weber, Journal of Physics-Condensed Matter 21 (2009) 435401.

[21] B. Dorado, B. Amadon, M. Freyss, M. Bertolus, Physical Review B 79 (2009) 235125.

[22] H.Y. Geng, Y. Chen, Y. Kaneta, M. Kinoshita, Physical Review B 75 (2007).

[23] I.D. Prodan, G.E. Scuseria, R.L. Martin, Physical Review B 76 (2007) 033101.

[24] J.E. Klepeis, Journal of Materials Research 21 (2006) 2979-2985.

[25] K.N. Kudin, G.E. Scuseria, R.L. Martin, Physical Review Letters 89 (2002)

[26] S.L. Dudarev, D.N. Manh, A.P. Sutton, Philosophical Magazine: Physics of condensed matter, statistical mechanics, electronic, optical, and magnetic properties 75 (1997) 613-628.

[27] V.A. Gubanov, A. Rosen, D.E. Ellis, Solid State Communications 22 (1977) 219223.

[28] B. Dorado, G. Jomard, M. Freyss, M. Bertolus, Physical Review B 82 (2010) 035114.

[29] P. Nerikar, T. Watanabe, J.S. Tulenko, S.R. Phillpot, S.B. Sinnott, Journal of Nuclear Materials 384 (2009) 61-69.

[30] H.Y. Geng, Y. Chen, Y. Kaneta, M. Iwasawa, T. Ohnuma, M. Kinoshita, Physical Review B 77 (2008).

[31] F. Gupta, G. Brillant, A. Pasturel, Philosophical Magazine 87 (2007) 2561-2569.

[32] M. Iwasawa, Y. Chen, Y. Kaneta, T. Ohnuma, H.Y. Geng, M. Kinoshita, Materials Transactions 47 (2006) 2651-2657. 
[33] J.P. Crocombette, F. Jollet, L.N. Nga, T. Petit, Physical Review B 64 (2001) 104107.

[34] E.D.A. Ferriss, R.C. Ewing, U. Becker, American Mineralogist 95 (2010) 229241.

[35] T. Watanabe, S.B. Sinnott, J.S. Tulenko, R.W. Grimes, P.K. Schelling, S.R. Phillpot, Journal of Nuclear Materials 375 (2008) 388-396.

[36] C.B. Basak, A.K. Sengupta, H.S. Kamath, The Journal of Alloys and Compounds 360 (2003) 210-216

[37] P.J.D. Lindan, M.J. Gillan, Journal of Physics-Condensed Matter 3 (1991) 39293939.

[38] B. Dorado, P. Garcia, G.e.l. Carlot, C. Davoisne, M. Fraczkiewicz, B. Pasquet, M. Freyss, C. Valot, G. Baldinozzi, D. Sim\&eacute;one, M. Bertolus, Physical Review B 83 (2011) 035126.

[39] F. Gupta, A. Pasturel, G. Brillant, Physical Review B 81 (2010) 014110.

[40] D.A. Andersson, T. Watanabe, C. Deo, B.P. Uberuaga, Physical Review B 80 (2009) 060101.

[41] K. Govers, S. Lemehov, M. Hou, M. Verwerft, Journal of Nuclear Materials 395 (2009) 131-139.

[42] H. Matzke, Journal of the Less-Common Metals 121 (1986) 537-564.

[43] Y. Yun, H. Kim, H. Kim, K. Park, Journal of Nuclear Materials 378 (2008) 40-44.

[44] B. Dorado, D.A. Andersson, C.R. Stanek, M. Bertolus, B.P. Uberuaga, G. Martin, M. Freyss, P. Garcia, Physical Review B 86 (2012) 035110.

[45] G. Kresse, J. Furthmuller, Vienna ab-initio simulation package, Universitat Wein, 2004.

[46] P.E. Blöchl, Physical Review B 50 (1994) 17953-17979.

[47] J.P. Perdew, Y. Wang, Physical Review B 45 (1992) 13244-13249.

[48] J.P. Perdew, J.A. Chevary, S.H. Vosko, K.A. Jackson, M.R. Pederson, D.J. Singh, C. Fiolhais, Physical Review B 46 (1992) 6671-6687.

[49] J.P. Perdew, Y. Wang, Physical Review B 33 (1986) 8800-8802.

[50] A.I. Liechtenstein, V.I. Anisimov, J. Zaanen, Physical Review B 52 (1995) R5467R5470.
[51] J. Schoenes, Journal of Applied Physics 49 (1978) 1463-1465.

[52] J. Wang, R.C. Ewing, U. Becker, Electronic Structure and Stability of Hyperstoichiometric $\mathrm{UO}_{2+x}$ Under Pressure, (in press).

[53] P. Santini, S. Carretta, G. Amoretti, R. Caciuffo, N. Magnani, G.H. Lander Reviews of Modern Physics 81 (2009) 807-863.

[54] H.Y. Geng, Y. Chen, Y. Kaneta, M. Kinoshita, Q. Wu, Physical Review B 82 (2010) 094106.

[55] B. Meredig, A. Thompson, H.A. Hansen, C. Wolverton, A. van de Walle, Physical Review B 82 (2010) 195128.

[56] F. Jollet, G. Jomard, B. Amadon, J.P. Crocombette, D. Torumba, Physical Review B 80 (2009) 235109.

[57] L.E. Cox, W.P. Ellis, R.D. Cowan, J.W. Allen, S.J. Oh, I. Lindau, B.B. Pate, A.J. Arko, Physical Review B 35 (1987) 5761-5765.

[58] S. Greaux, L. Gautron, D. Andrault, N. Bolfan-Casanova, N. Guignot, J. Haines, American Mineralogist 93 (2008) 1090-1098.

[59] F. Birch, Physical Review 71 (1947) 809-824.

[60] M. Idiri, T. Le Bihan, S. Heathman, J. Rebizant, Physical Review B 70 (2004) 014113.

[61] U. Benedict, G.D. Andreetti, J.M. Fournier, A. Waintal, Journal De Physique Lettres 43 (1982) L171-L177.

[62] M. Mantina, Y. Wang, L.Q. Chen, Z.K. Liu, C. Wolverton, Acta Materialia 57 (2009) 4102-4108.

[63] S. Lany, A. Zunger, Physical Review B 78 (2008) 235104

[64] M.T. Weller, P.G. Dickens, D.J. Penny, Polyhedron 7 (1988) 243-244.

[65] C.G. Van de Walle, J. Neugebauer, Journal of Applied Physics 95 (2004) 3851 3879.

[66] F.N. Skomurski, J.W. Wang, R.C. Ewing, U. Becker, Journal of Nuclear Materials (in press). doi: 10.1016/j.jnucmat.2011.1009.1003.

[67] D. Sheppard, R. Terrell, G. Henkelman, Journal of Chemical Physics 128 (2008) 134106.

[68] N.N. Greenwood, A. Earnshaw, Chemistry of the Elements, second ed., Butterworth-Heinemann, Oxford, 1997. 


\title{
He diffusion and closure temperatures in apatite and zircon: A density functional theory investigation
}

\author{
Amelia Bengtson*, Rodney C. Ewing, Udo Becker \\ Department of Geological Sciences, University of Michigan, Ann Arbor, MI 48109-1005, United States
}

Received 4 August 2011; accepted in revised form 3 March 2012; available online 14 March 2012

\begin{abstract}
Diffusion of $\mathrm{He}$ in zircon and apatite is of fundamental importance in the interpretation of He-loss measurements used in thermochronology. The diffusion of $\mathrm{He}$ in zircon is strongly anisotropic, while experimental measurements find He diffusion in apatite to be nearly isotropic. We present the first calculations for He diffusion in $\mathrm{Ca}_{5}\left(\mathrm{PO}_{4}\right)_{3} \mathrm{~F}$ fluroapatite and re-calculate He diffusivity in zircon, $\mathrm{ZrSiO}_{4}$, in order to make a consistent comparison with the results of the apatite calculations and clarify discrepancies in the literature. Calculated diffusivities for apatite are:

$$
\begin{aligned}
& D_{\left[\begin{array}{ll}
01 \\
0
\end{array}\right]}\left(\mathrm{cm}^{2} / \mathrm{s}\right)=0.014 \exp \left(-84 \mathrm{~kJ} \mathrm{~mol}^{-1} / R T\right) \\
& D_{\left[\begin{array}{ll}
1 & 10]
\end{array}\right]}\left(\mathrm{cm}^{2} / \mathrm{s}\right)=0.024 \exp \left(-104 \mathrm{~kJ} \mathrm{~mol}^{-1} / R T\right)
\end{aligned}
$$

and for zircon:

$$
\begin{aligned}
& D_{\left[\begin{array}{ll}
011 \\
\text { 1 }
\end{array}\right.}\left(\mathrm{cm}^{2} / \mathrm{s}\right)=0.0039 \exp \left(-42 \mathrm{~kJ} \mathrm{~mol}^{-1} / R T\right) \\
& D_{\left[\begin{array}{ll}
100] \\
0
\end{array}\right.}\left(\mathrm{cm}^{2} / \mathrm{s}\right)=0.030 \exp \left(-255 \mathrm{~kJ} \mathrm{~mol}^{-1} / R T\right)
\end{aligned}
$$

He diffusion in ideal zircon is greater than in ideal apatite and anisotropic in both. However, the degree of anisotropy is much more pronounced in zircon. The computational approach allows a comparison of the behavior of the ideal structures (i.e., defect-free) as compared to natural samples that may contain impurities or some level of radiation damage. The calculated diffusivities for the ideal structure are in closer agreement with experimentally determined values for natural apatite than for zircon. The calculations predict that the perfect zircon structure will have high diffusivities due to large uninterrupted "channels" along [001]. However, in natural samples, these channels may be interrupted due to the presence of impurities, e.g., radiogenic $\mathrm{Pb}$, or nanoscale radiation-damage cascades, $5 \mathrm{~nm}$ in diameter, created by the alpha-decay of incorporated $\mathrm{U}$ and $\mathrm{Th}$, thus effectively lowering the diffusivity. The damage microstructure depends on the fluence and thermal history of the sample, and variations in thermal history can lead to variations in the He-loss and the interpreted age and thermal history. Closure temperatures in the ideal structure are extremely low, $-35^{\circ} \mathrm{C}$ for apatite and $-150{ }^{\circ} \mathrm{C}$ for zircon, suggesting the degree of radiation damage plays an important role in attaining closure to He loss.
\end{abstract}

(C) 2012 Elsevier Ltd. All rights reserved.

\section{INTRODUCTION}

Thermochronology is typically used to determine the age and thermal history of rocks within the Earth's crust

\footnotetext{
* Corresponding author.

E-mail address: akbengtson@wisc.edu (A. Bengtson).
}

(Ehlers and Farley, 2003; Ehlers, 2005; Reiners et al., 2005). Apatite and zircon are the most common minerals used in (U-Th)/He thermochronometry (Ehlers and Farley, 2003; Thomson et al., 2010). The age of a mineral can be determined from the ratio of parent nuclides, $\mathrm{U}$ and $\mathrm{Th}$, to their decay products, $\mathrm{Pb}$ and $\mathrm{He}$, assuming this ratio has not been disturbed by alteration or thermal events. 
However, in the (U-Th)/He system, helium can easily be lost by thermally-activated diffusion, resulting in lower measured ages, but also providing information on the timing of thermal events (Reiners and Shuster, 2009). Therefore, a knowledge of the mechanisms and rates of loss of He are essential to interpreting the age and thermal histories of crustal rocks correctly, especially for minerals with anisotropic diffusion (Huber et al., 2011).

In order to extract accurate ages and thermal histories from apatite and zircon, the He loss within these minerals must be well constrained. In zircon, previous experimental and computational studies have shown diffusivity to be strongly anisotropic (Farley, 2007; Reich et al., 2007; Cherniak et al., 2009; Saadoune and de Leeuw, 2009; Saadoune et al., 2009). Experiments indicate He diffusion in apatite is almost isotropic and greater (faster) than in zircon (Farley, 2000; Cherniak et al., 2009). In this study, the transport properties of He are calculated in ideal apatite at the atomic scale using density functional theory (DFT) methods. This approach, using the ideal atomic-scale structure in the absence of impurities and without radiation damage, provides a benchmark for the comparison of diffusion rates among structures and leads to predictions of He loss. Experimental values are obtained from crystals that may have defects or impurities, some level of radiation damage and different thermal histories. The DFT calculations in this paper focus on the determination of the activation energy, $E_{A}$, the diffusion prefactor, $D_{0}$, and closure temperature, $T_{C}$, as a function of crystallographic direction for different He diffusion pathways through the structures. The activation barriers in ideal zircon have been previously calculated (Reich et al., 2007; Saadoune and de Leeuw, 2009; Saadoune et al., 2009). Here, $E_{A}$ is recalculated for zircon in order to compare the results for apatite to zircon using the same computational methods, understand discrepancies in previous studies (Reich et al., 2007; Saadoune and de Leeuw,
2009; Saadoune et al., 2009), and incorporate results into estimates of closure temperatures.

At the atomic level, diffusion is the hop of one He atom from a minimum energy position to the next. The defect model used assumes He diffuses interstitially from one He minimum to another, which is often the case for cation diffusion. Other mechanisms include coupled substitution with others atoms or vacancies (Watson and Baxter, 2007). DFT calculations were used to calculate the activation energy barrier, $E_{A}$, for an atom hopping between He minima and the vibrational modes associated with the hop. Using the actual vibrational modes for the calculation of $D_{0}$ is a more advanced model than deriving $D_{0}$ just from $E_{A}$ as done in Reich et al. (2007), as explained in Section 2.3. From $E_{A}$, models for diffusion and closure temperature were calculated.

Qualitative diffusion behavior can be inferred simply by examining the main features of the ideal structure (Fig. 1). The zircon structure has large continuous channels in the $\left[\begin{array}{lll}0 & 0 & 1\end{array}\right]$ direction. In contrast, along [0 0101$]$ the apatite structure is blocked by intervening $\mathrm{Ca}$ atoms. Thus, greater $\mathrm{He}$ diffusion is expected along [001] in zircon than apatite, in contrast to the results of laboratory measurements.

\section{METHODS}

Density functional theory (DFT) calculations solve the Schrödinger equation, the governing equation of quantum mechanics. The Schrödinger equation is comprised of an energy operator, the Hamiltonian operator, and the wave function (also know as the quantum state); together these describe the fundamental quantum physics of a given system. The Schrödinger equation cannot be solved exactly for a many-electron system. DFT expresses the Schrödinger equation as a function of the electron density, in which all terms can be written and solved explicitly, except for the electron exchange-correlation functional. Exchange correlation

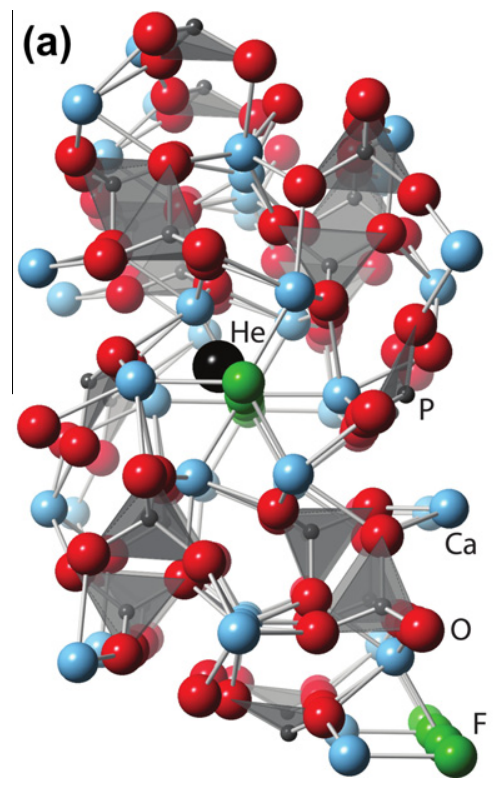

(b)

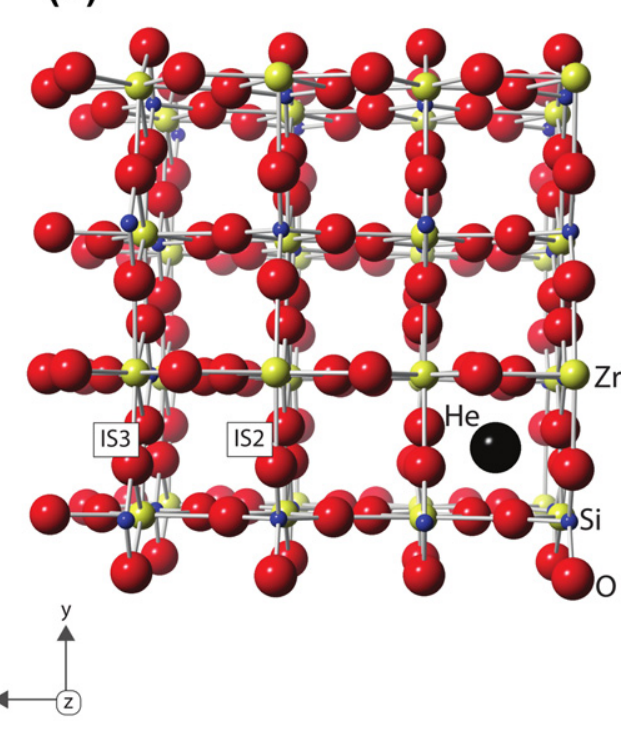

Fig. 1. (a) Apatite and (b) zircon structures. [001] is perpendicular to the page. The atoms shown are representative of the unit cells used in the calculations. Periodic boundary conditions are used in the calculations, thereby creating an extended model of the bulk, crystalline material. 
describes how electrons interact at the quantum level and is typically approximated with either a local density approximation (LDA) or a generalized gradient approximation (GGA). This simpler form of the Schrödinger equation is tractable to solve for real systems (Sholl and Steckel, 2009). As input, DFT calculations require knowledge of the crystal structure and composition. DFT calculations output ground-state energies, densities and wave functions, which can then be used to obtain macroscopic properties (Hafner et al., 2006). DFT calculations are independent of experimental constraints and experimental data, therefore results obtained from DFT can be compared independently with experimental results. For further reading regarding density functional theory in modern research, please see the review by Hafner et al. (2006).

The Vienna $\mathrm{Ab}$ Initio Simulation Package (VASP) (Kresse and Hafner, 1993; Kresse and Furthmuller, 1996a,b) was used for these DFT calculations. The Generalized Gradient Approximation (GGA) with the PerdewBurke-Ernzerhof (PBE) parameterization (Perdew et al., 1996) was used to approximate the exchange-correlation with planewave energy cutoff of $600 \mathrm{eV}^{1}$. The planewaves serve as basis functions for the simulation of the wave function. Structural relaxations were performed using full geometry optimization with the conjugate gradient relaxation technique.

The DFT approximation is only valid for the ground state of a system, therefore the thermal motion of the lattice atoms is not considered in the calculations, except for the diffusing atom, as described by equation 1 in Section 2.3. The overall impact of the atomic vibrations on the He energy barriers should be small. A static-lattice approach for DFT calculations of transport properties has been widely used in the literature (Van der Ven et al., 2001, 2008; Van der Ven and Ceder, 2005; Saadoune et al., 2009; Ammann et al., 2010).

\subsection{Structure}

Calculations were performed for $\mathrm{Ca}_{5}\left(\mathrm{PO}_{4}\right)_{3} \mathrm{~F}$ fluroapatite (hexagonal space group 176: $P \sigma_{3} / \mathrm{m}$ ). Periodic boundary conditions were used; therefore, the structure is repeated infinitely in all directions representing a pure crystalline bulk with repeating $\mathrm{He}$ atoms separated by the spacing of the different superlattices. A 168-atom orthogonal [1 $00 ; 120 ; 002]$ supercell (Fig. 1a) with $a, b, c$ unit cell parameters 9.464, $16.393,13.843 \AA$ and angles $90^{\circ}, 90^{\circ}, 90^{\circ}$ was large enough to avoid $\mathrm{He}-\mathrm{He}$ interactions (from $\mathrm{He}$ atoms in neighboring cells) and to represent dilute He concentrations. The atomic positions in the supercell were fully relaxed. All diffusion calculations containing He were performed with $P_{1}$ symmetry, which allowed for potentially different diffusion constants of He in different crystallographic directions.

Determining diffusion pathways is a two-step process. First, the most energetically favorable locations or local min-

\footnotetext{
${ }^{1}$ The pseudopotentials used were generated in VASP. For reproducibility, the dates of generation are $\mathrm{Ca}$ : 06 Sep 2000, P: 17 Jan 2003, O: 08 Apr 2002, F: 08 Apr 2002, Zr: 08 Apr 2002, Si: s2p2, O (zircon): 07 Sep 2000, He: 05 Jan 2001.
}

ima of helium $(\mathrm{He})$ within the cell must be found. Then, pathways are identified from one local minimum He position to the next symmetrically equivalent minimum. Few data exist in the literature on the interstitial location for $\mathrm{He}$ in apatite. In this study, the minimum energy position for interstitial $\mathrm{He}$ was found by identifying five possible interstitial sites for $\mathrm{He}$ (Fig. 2a). The structure was relaxed and the energy minimized for all five $\mathrm{He}$ sites, in independent calculations with one $\mathrm{He}$ atom/cell. Based on these results, the most energetically favorable locations for a $\mathrm{He}$ atom are close to the axis that connects the F atoms along [0 01 ] (site 1 and site 2; Fig. 2a). Sites 3, 4, and 5 (local minima) are not as energetically favorable as sites 1 and 2 (global minima), therefore diffusion paths in Section 2.2 are only considered to and from site 2 (symmetrically equivalent to site 1 ).

The 192-atom $3 \times 3 \times 3$ supercell of zircon, $\mathrm{ZrSiO}_{4}$, (space group 141: $I 4_{1} / a m d$ ) used in the calculations had $a$, $b$, and $c$ unit cell parameters of 13.370, 13.370, and $12.059 \AA$ with angles of $90^{\circ}, 90^{\circ}$, and $90^{\circ}$. The minimum energy position for $\mathrm{He}$ in zircon (Fig. 1b) was selected based on previous studies (Reich et al., 2007; Saadoune and de Leeuw, 2009; Saadoune et al., 2009).

\subsection{Calculating activation barriers}

The activation barrier, $E_{A}$, is the energy required to move a $\mathrm{He}$ atom from one minimum energy position (site 1 or site 2; Fig. 2a) along a given direction in the structure through activated states to the next minimum energy position. Specifically, $E_{A}$ is the energy difference between $\mathrm{He}$ in the activated state and in the minimum energy position. Potential pathways for He diffusion were identified from the $\mathrm{He}$ site (site 2 ) to the next symmetrically equivalent site (e.g., site $2^{*}$ in Fig. $2 \mathrm{~b}$ and site 1 in Fig. 2c). Interstitial diffusion requires open channels through the crystal structure. Close inspection of the structure reveals two potential pathways for interstitial diffusion, along [001] (Fig. 2b) and [110] (Fig. 2c). These are the two most open channels in apatite and therefore the most probable directions of movement. Pathways from site 2 in other directions are too congested with atoms to make interstitial diffusion likely. In zircon, $E_{A}$ along [ 0001$]$ and [ 100$]$ ] was calculated; consistent with previous literature (Reich et al., 2007; Saadoune and de Leeuw, 2009; Saadoune et al., 2009).

The activation barrier, $E_{A}$, was calculated in previous zircon studies by manually placing the $\mathrm{He}$ atom at even intervals along the path between two energy minima (Reich et al., 2007; Saadoune and de Leeuw, 2009; Saadoune et al., 2009). Using this method, the coordinates of the He atom are fixed at each respective position along the path (though $\mathrm{He}$ is allowed to relax perpendicular to the path along with all of the other atoms in the cell) in order to avoid atoms relaxing into the local minimum position, and the energy is calculated for each step. For some cells, an additional atom, far from the $\mathrm{He}$ atom is fixed to avoid drift of the unit cell during the calculation. Since the He position is not allowed to fully relax during the energy calculation, the activated energy may be artificially high or low. Zircon has evenly spaced barriers in a grid-like pattern that make manually placing $\mathrm{He}$ atoms along the energy barrier path 
(a) Possible interstitial sites for $\mathrm{He}$

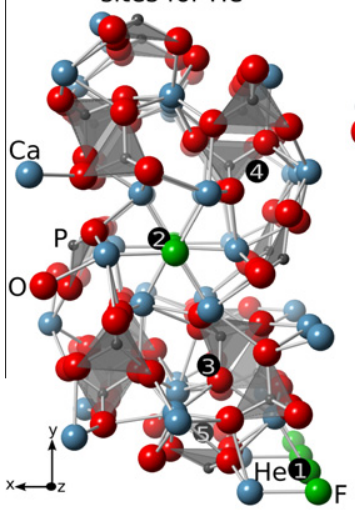

(b) Pathway 1

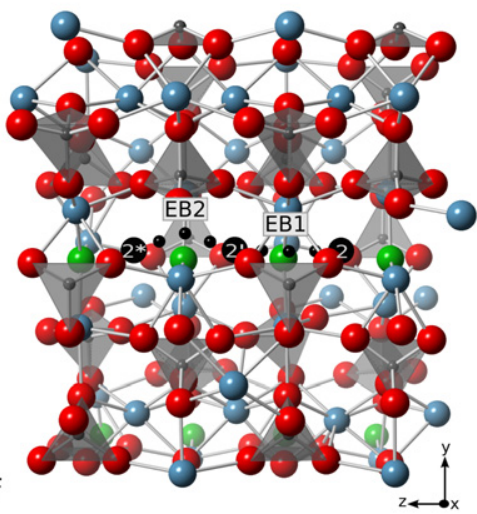

(c) Pathway 2

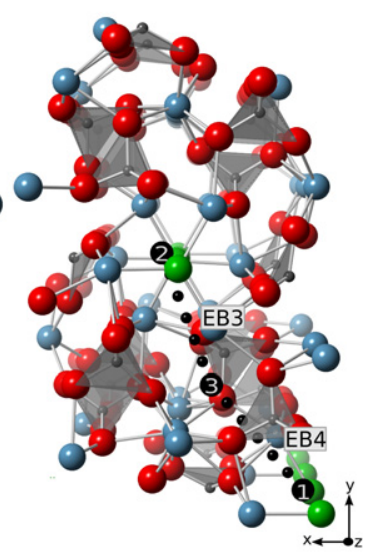

(d) Barriers along Pathway 1

(e) Barriers along Pathway 2
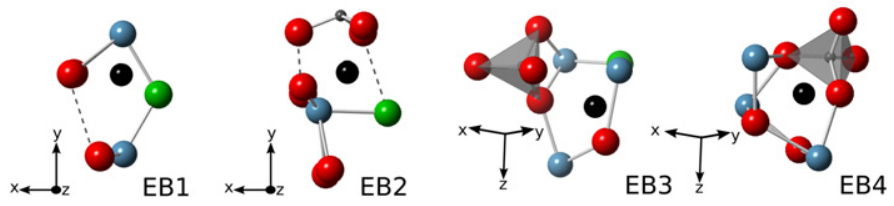

Fig. 2. $169 \mathrm{He}-\left(\mathrm{Ca}_{5}\left(\mathrm{PO}_{4}\right)_{3} \mathrm{~F}\right)_{8}$ apatite supercell used in calculations. (a) Five possible interstitial sites for He were identified (labeled 1 through 5). Based on the results of the calculations, site 2 (equivalent to site 1) is the energetically most favorable site for He. From site 2, potential pathways for He diffusion are identified in all directions. (b) Pathway 1 is along the F column in the [001] direction. He travels from site 2 to the next symmetrically equivalent He site, $2^{*}$, through barriers EB1 and EB2 (d). (c) Pathway 2 is along the [1 10] direction from site 2 to the symmetrically equivalent site 1 via barriers EB3 and EB4 and site 3 (e). In (b) and (c), the large black atoms 2, $2^{*}$ and 1 are global minimum energy positions for $\mathrm{He}$, large black atoms $2^{\prime}$ and 3 are local minimum energy positions, and the smaller black atoms illustrate the activated and intermediate positions for He along the pathway. Although cells are shown with multiple He atoms to illustrate potential He interstitial sites and diffusion pathways, all calculations were performed with one He atom/cell. Barriers (d and e), especially along [1 10$]$ (e) are threedimensional and the axes have been rotated for clarity. Dashed lines fill in the geometric boundary.

fairly obvious (Fig. 1). The complexities of apatite's structure calls for a more sophisticated method of calculating the energy barrier.

Another approach to calculate $E_{A}$ is the Nudged Elastic Band (NEB) method, which allows calculation of the energy of $\mathrm{He}$ in the activated state without fixing the position (Mills et al., 1995; Jónsson et al., 1998). The initial setup is similar to the method described above: unit cells are created with He atoms placed at even intervals between the two energy minima. Each unit cell contains only one $\mathrm{He}$ atom. The NEB method connects the atoms in different unit cells along the path with an imaginary spring. The spring-connected atoms, referred to as images, keep He in the activated state, preventing it from relaxing into the local minimum position. The energy is calculated for all images along the path simultaneously, allowing $\mathrm{He}$ in the activated state to relax fully in all directions to find the lowest activation barrier. Images were equidistant and the center of mass is the same for all images (so the cells do not drift during the calculation). All NEB calculations were run with a spring constant of $-5 \mathrm{eV} / \AA^{2}$. Scripts were used to create the images at equidistant spacing with the same center of mass (Curtarolo et al., 2005). The NEB method was used in this study to calculate barriers for both apatite and zircon.

In apatite, pathway 1 is along [001] from site 2 to the next symmetrically equivalent He location, $2^{*}$, through barriers EB1 and EB2 (Fig. 2b, 2d). He in site $2^{\prime}$ has the same energetics as in site 2, however 2' is not symmetrically equivalent to site 2 . Both barriers are approximately diamond-shaped (Fig. 2d). For the NEB calculations, results are reported for one image between 2 and $2^{\prime}$ and one image between 2' and 2*. Using three images (as shown in Fig. 2) did not change the activation barrier.

Pathway 2 is along [110] from site 2 to the next symmetrically equivalent global $\mathrm{He}$ minimum location, site 1 (Fig. 2c). He travels from site 2 to site 3 through barrier EB3 and from site 3 to site 1 through EB4 (Fig. 2e). As discussed in Section 2.1, He in site 3 is a local minima, not a global minima and is not as energetically favorable as sites 1 or 2 (Fig. 3a). The barriers along [110] resemble three-dimensional cages. For the NEB calculations, 5 images were used between sites 2 and 3 and 5 images were used between sites 1 and 3 for a total of 10 images along the path.

\subsection{Calculating diffusivities}

Tracer diffusion, $D^{*}$, describes the path (aka trace) of the motion of a single particle (Kutner, 1981; Gomer, 1990) and is a function of $E_{A}$ :

$D^{*}=D_{0} \exp \left(\frac{-E_{A}}{R T}\right)$ 

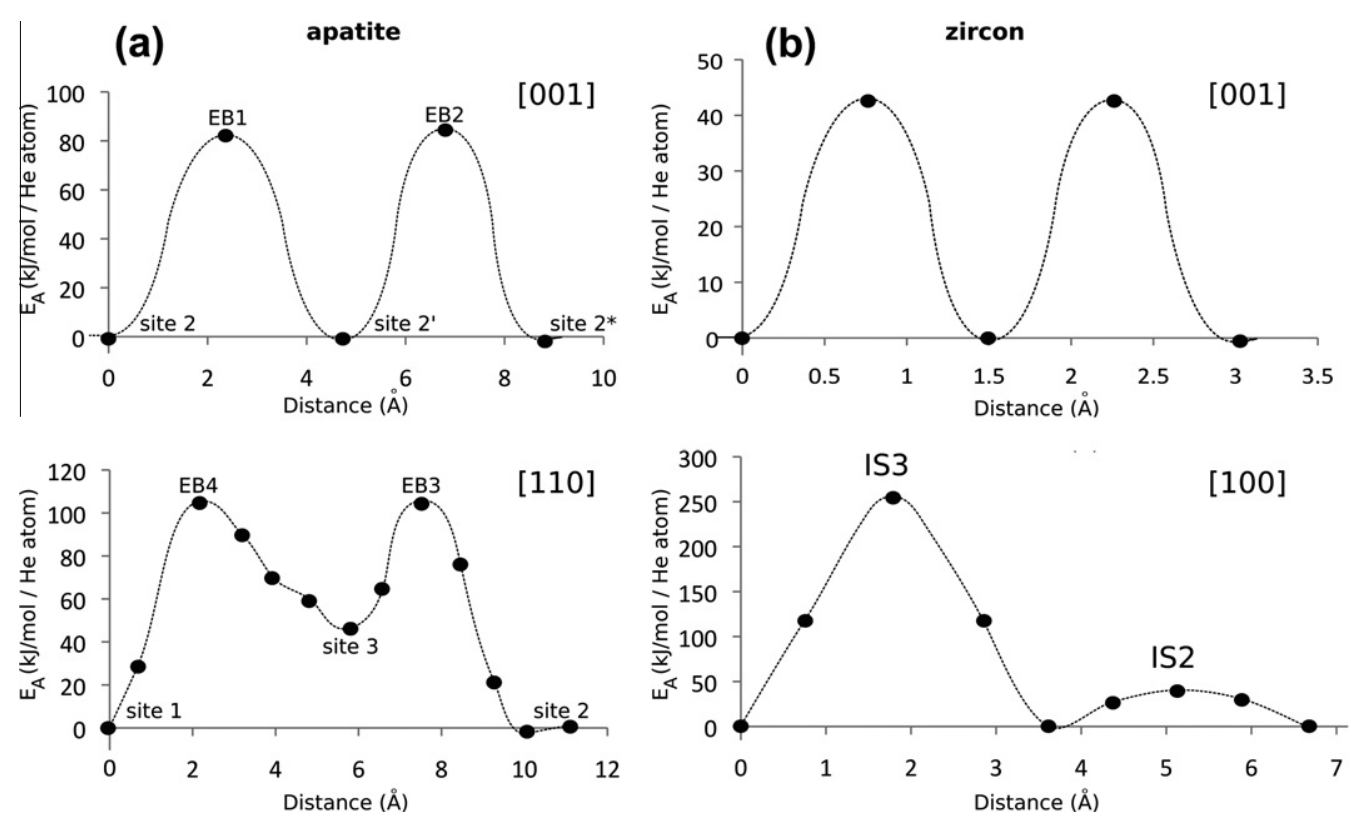

Fig. 3. Energy for each image relative to the minimum energy position in (a) apatite along [001] and [1 10$]$ and (b) zircon along the [00 1] and $[100]$. Sites 1,2 , and 3 are defined in Fig. 2. The activation energy $\left(E_{A}\right)$ for each path is the largest energy along that path. Note, that the vertical scale for $E_{A}$ varies considerably for each figure. EB1-EB4 are the barriers in apatite, shown in Fig. 2. IS2 is an octahedral-shaped interstitial site and IS3 is a smaller diamond-shaped interstitial site (geometry shown in Fig. 3 of (Saadoune et al., 2009)).

$D_{0}=\frac{z a^{2}}{2 d} v$,

where $z$ is the coordination of the atom, $a$ is the hop-distance between two He minima, $d$ is the dimension ( 3 for all calculations considered here), $R$ is the gas constant, and $T$ is the temperature. $E_{A}$ is calculated with the DFT method using the formulas above $(1,2)$. All atoms vibrate at a natural frequency within a crystal. This vibration is the driving force that moves an atom from one point in the structure to another. The attempt frequency (Vineyard, 1957), $v$, was calculated from normal modes of vibration in the DFT calculation by displacing the atom in the activated and ground states. The attempt frequency is the ratio of $v_{i}$ (the normal modes of the stable state) to $v_{i}{ }^{*}$ (the normal modes of the activated state) for $M \mathrm{He}$ atoms (1 in this case) (Allnatt and Lidiard, 1993).

$$
v=\frac{\prod_{i}^{3 M} v_{i}}{\prod_{i}^{3 M-1} v_{i}^{*}}
$$

In VASP, $v_{i}$ and $v_{i}^{*}$ are calculated by slightly displacing the $\mathrm{He}$ atom in all directions at the stable and activated sites. Calculating normal modes with DFT allows direct determination of $v$ from first principles with the only approximation being those made in DFT to solve the Schrödinger equation. Another way to approximate $v$ is to write the frequency as a function of the force constant and assume the potential function is represented by a one-dimensional sinusoidal function $\phi(x)=\phi(0)+E \sin ^{2}$ $\left(\frac{\pi x}{\lambda}\right)$ (Reich et al., 2007; Saadoune and de Leeuw, 2009;
Saadoune et al., 2009). Although simple structures may have a fairly sinusoidal potential function, the shape of apatite's potential curve is more complex and not accurately represented by a one-dimensional sine function (Fig. 3).

Chemical diffusion, $D$, is the product of the self-diffusion term, $D_{J}$, with the thermodynamic factor, $\Theta$, for the system: $D=D_{J} \cdot \Theta$. The self-diffusion term $D_{J}$ is proportional to the mean squared displacement of the atoms of interest in the system (Van der Ven et al., 2008).

In the dilute limit, such as in these calculations ( 0.6 at. $\%$ He in apatite separated by $9.5 \AA, 0.5$ at.\% He in zircon separated by $12.06 \AA$ ), one can assume that $D_{J}=D^{*}$ because there are no cross-correlations between He atoms; thus, $\Theta$ is 1 . Therefore, for the dilute limit, the chemical diffusion is the same as the tracer diffusion $\left(D=D^{*}\right.$ ) (Kutner, 1981; Gomer, 1990; Van der Ven et al., 2008).

\section{RESULTS}

\subsection{Activation barriers}

The lowest activation barrier in apatite is along [0 011$]$ (Table 1, Table 2, Fig. 3) at $84 \mathrm{~kJ} / \mathrm{mol}$. Calculations also clearly show anisotropy in the activation barriers: $E_{A}$ in the [110] direction is $20 \mathrm{~kJ} / \mathrm{mol}$ higher than in the [001] direction.

The lowest activation barrier $(42 \mathrm{~kJ} / \mathrm{mol})$ in zircon is along [ $\left.\begin{array}{ll}0 & 0\end{array}\right]$ (Table 1, Fig. 4a, solid black diamonds). Calculations also show strong anisotropy in the activation barriers; the activation barrier for [100] is $213 \mathrm{~kJ} / \mathrm{mol}$ higher than for the [001]. Energy barriers are lowest along [0 011$]$ for both apatite and zircon. In zircon, $E_{A}$ differs by 
Table 1

Diffusivity parameters in apatite and zircon as a function of direction in the structure. $a$ : hop distance, $E_{A}$ : activation energy, $v$ : attempt frequency, $D_{0}$ : diffusivity. The atomic coordination, $z$, is 4 for all pathways.

\begin{tabular}{|c|c|c|c|c|c|c|}
\hline & & $a(\AA)$ & $E_{A}(\mathrm{~kJ} / \mathrm{mol})$ & $E_{A}(\mathrm{eV})$ & $v(\mathrm{THz})$ & $D_{0}\left(\mathrm{~cm}^{2} / \mathrm{s}\right) \times 10^{-2}$ \\
\hline \multirow[t]{2}{*}{ Apatite } & {$\left[\begin{array}{lll}0 & 0 & 1\end{array}\right]$} & 8.8 & 84 & 0.87 & 2.79 & 1.4 \\
\hline & {$\left[\begin{array}{lll}1 & 1 & 0\end{array}\right]$} & 11.09 & 104 & 1.08 & 2.90 & 2.4 \\
\hline \multirow[t]{4}{*}{ Zircon } & {$\left[\begin{array}{lll}0 & 0 & 1\end{array}\right]$} & 3.02 & 42 & 0.44 & 6.36 & 0.39 \\
\hline & {$\left[\begin{array}{lll}1 & 0 & 0\end{array}\right]-$ IS2 } & 3.06 & 42 & 0.43 & 5.02 & 0.31 \\
\hline & {$\left[\begin{array}{lll}1 & 0 & 0\end{array}\right]-$ IS3 } & 3.59 & 255 & 2.64 & 10.06 & 0.86 \\
\hline & {$\left[\begin{array}{lll}1 & 0 & 0\end{array}\right]$ - Total } & 6.65 & 255 & 2.64 & 10.06 & 3.0 \\
\hline
\end{tabular}

Table 2

Comparison of calculated and measured activation energies for apatite. Bulk step-wise heating measurements are interpreted as isotropic diffusivities (Wolf et al., 1996; Farley, 2000; Shuster and Farley, 2005). Ion beam measurements show a slight anisotropy, (Cherniak et al., 2009). Measurements report barriers along [0 011$]$ and normal to [0 011$]$. Calculated activation energies are along [0 011$]$ and [1 10$]$.

\begin{tabular}{llll}
\hline & $\begin{array}{l}\text { Bulk (isotropic) activation } \\
\text { energy }(\mathrm{kJ} / \mathrm{mol})\end{array}$ & $\begin{array}{l}\text { Activation energy along } \\
{[001](\mathrm{kJ} / \mathrm{mol})}\end{array}$ & $\begin{array}{l}\text { Activation energy along }[110] \\
\text { (normal to }[001](\mathrm{kJ} / \mathrm{mol})\end{array}$ \\
\hline $\begin{array}{l}\text { This work - DFT } \\
\begin{array}{l}\text { Ion beam measurements (Cherniak } \\
\text { et al., 2009) }\end{array}\end{array}$ & - & 84 & 104 \\
$\begin{array}{l}\text { Step-wise heating measurements } \\
\text { (Shuster and Farley, 2005) }\end{array}$ & 124 & $129 \pm 12$ & - \\
$\begin{array}{l}\text { Step-wise heating measurements } \\
\text { (Farley, 2000) }\end{array}$ & $138 \pm 2$ & - & - \\
$\begin{array}{l}\text { Step-wise heating measurements (Wolf } \\
\text { et al., 1996) }\end{array}$ & 151 & - & - \\
\hline
\end{tabular}

\section{(a)}

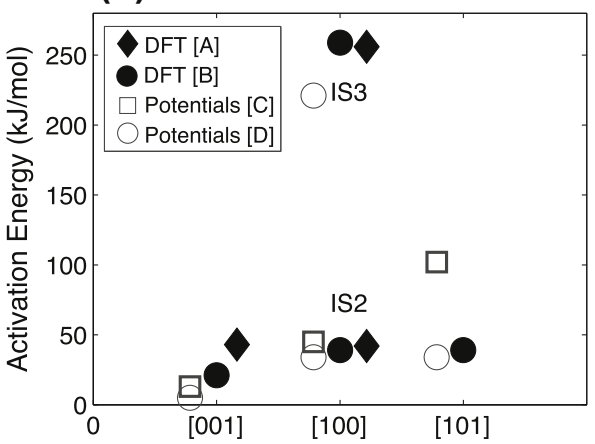

(b)

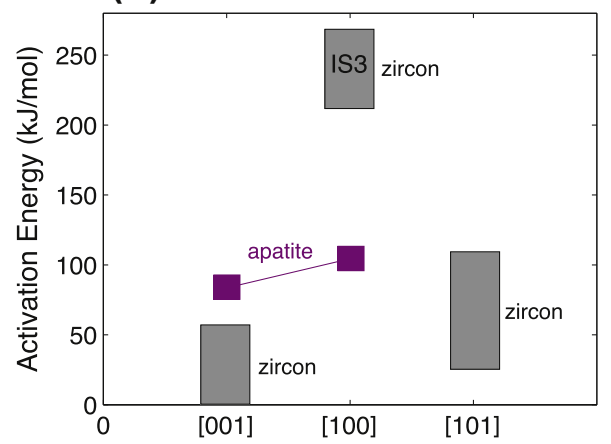

Fig. 4. (a) Computational activation barriers in zircon from this work and the literature. DFT work represented by solid markers, empirical potential work represented by open markers. [A, filled diamonds] $=$ this work, DFT; shifted to right for clarity. [B, filled circles] $=$ DFT work by Saadoune et al. (2009)). [C, open squares]= empirical potential work by Reich et al. (2007); shifted to left for clarity. [D, open circles] = empirical potential work by Saadoune et al. (2009)); shifted to left for clarity. Along [100], there are two different barriers for He mobility. IS2 is an octahedral-shaped interstitial site and IS3 is a smaller diamond-shaped interstitial site (geometry shown in Fig. 3 of (Saadoune et al., 2009)). (b) Summary of the ranges of $E_{A}$ plotted in (a). IS2 and IS3 are two barriers along the same diffusion pathway. IS3 has the highest activation barrier and is the true barrier to mobility along [100], therefore IS2 is not shown in (b). $E_{A}$ values of apatite, calculated with DFT (Tables 1 and 2), are plotted in (b) for comparison with zircon.

$200 \mathrm{~kJ} / \mathrm{mol}$ depending on direction, but for apatite the variation is only about $20 \mathrm{~kJ} / \mathrm{mol}$ (Table 1). Along $[001] E_{A}$ in apatite is clearly larger than in zircon (Fig. 4b).

\subsection{Diffusivities}

The diffusivities of He in apatite (Eqs. (1)-(3), Table 1) as a function of temperature are:

$$
\begin{aligned}
D_{[001]} & =0.014 \exp \left(-84 \mathrm{~kJ} \mathrm{~mol}^{-1} / R T\right) \mathrm{cm}^{2} / \mathrm{s} \\
D_{[110]} & =0.024 \exp \left(-104 \mathrm{~kJ} \mathrm{~mol}^{-1} / R T\right) \mathrm{cm}^{2} / \mathrm{s}
\end{aligned}
$$

In zircon, the diffusivities of $\mathrm{He}$ as a function of temperature are:

$$
\begin{aligned}
& D_{[001]}=0.0039 \exp \left(-42 \mathrm{~kJ} \mathrm{~mol}^{-1} / R T\right) \mathrm{cm}^{2} / \mathrm{s} \\
& D_{[100]}=0.030 \exp \left(-255 \mathrm{~kJ} \mathrm{~mol}^{-1} / R T\right) \mathrm{cm}^{2} / \mathrm{s}
\end{aligned}
$$



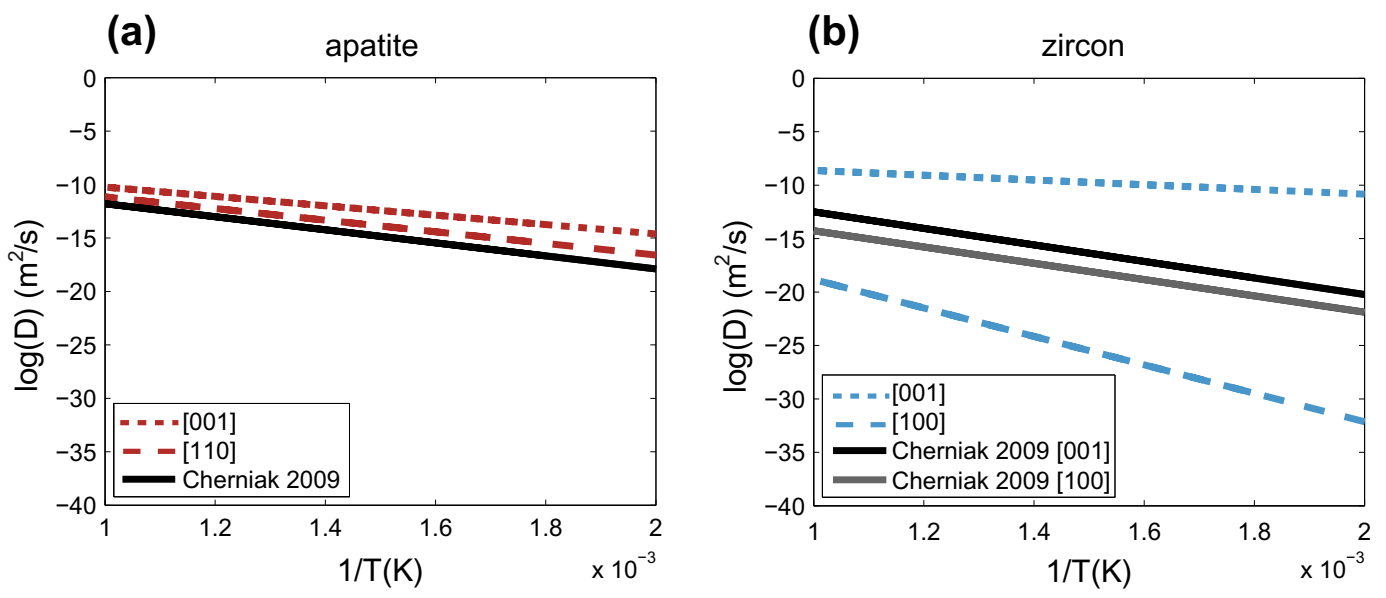

Fig. 5. Diffusivity as a function of temperature for He in (a) apatite and (b) zircon. DFT results from this work in [001] (small dashed line) and normal to [001] (large dashed line) are plotted with experimental ion beam measurements (solid lines, Cherniak 2009 (Cherniak et al., 2009)).

The above diffusivities are plotted in Fig. 5 for apatite (a) and zircon (b). For apatite, the calculated diffusivities are greater than the measured diffusivity (Cherniak et al., 2009) by 1-3 orders of magnitude, depending on direction. Diffusivity is anisotropic; diffusion for [001] is greater than $\left[\begin{array}{lll}1 & 1 & 0\end{array}\right]$ by over an order of magnitude.

The calculated diffusivities for He diffusion in zircon along the [0 011$]$ are also greater than the measured diffusivities (Fig. 5b). Diffusivity is anisotropic in both calculations and measuremens (Cherniak et al., 2009). The calculated diffusivity along [001] is 10-20 order of magnitude greater than along [100]; in contrast, measured diffusivities along different directions only vary by less than 3 orders of magnitude (Cherniak et al., 2009).

\section{DISCUSSION}

\subsection{Comparison of computational results}

For zircon, previous activation barrier calculations have been calculated (Reich et al., 2007; Saadoune and de Leeuw, 2009; Saadoune et al., 2009) and are plotted with the results from this study in Fig. 4a. Care must be taken when comparing calculations performed with different methods. Empirical potential methods and DFT solve completely different equations. As described in Section 2, DFT calculations solve an approximation of the Schrödinger equation and are independent of experimental constraints. While DFT calculations describe electronic interactions, classical force-field methods only deal with the interactions of rigid ions. Empirical potential methods (molecular statics or classical force-fields or molecular-dynamics) are based on solving Newton's equations of motion; atomic interactions are described by potentials that were fit to experimental or quantum-mechanical data. Results can be biased depending on the data used for fitting empirical potentials, and different potentials can lead to very different results. The benefit of empirical potential methods is that they are much faster than DFT calculations, can handle a large number of atoms, and allow for visualization of the diffusion process.
Additionally, molecular-dynamics simulations are run at finite temperature and include the thermal motion of all particles (diffusing and non-diffusing).

Overall, there is good agreement between this work and the previous DFT study of zircon (Saadoune et al., 2009b). One discrepancy is that $E_{A}$ along [001] from this work is $\sim 20 \mathrm{~kJ} / \mathrm{mol}$ higher than of Saadoune et al. (2009). Possible explanations include different choices of the computational parameters, such as the exchange-correlation approximation, and using the NEB method for calculating $E_{A}$. The latter explanation can be excluded because manually fixing He positions along the diffusion path (Saadoune et al., $2009 b$ ), as described in the methods section, yielded the same $E_{A}$ as our $E_{A}$ calculated with the NEB method. Overall, the computational differences along [ 0001 l 1 are not as significant as the differences in $E_{A}$ along different crystallographic directions.

The ideal zircon structure has two successive barriers to He mobility along [ 100$]$ between the two absolute minima, one is an octahedral-shaped interstitial site (IS2), and the other is a smaller diamond-shaped interstitial site (IS3). $E_{A}$ is higher through IS3 than IS2, thus IS3 is the true barrier (diffusion-limiting factor) to mobility along [100]. The DFT calculations and the empirical potential methods for zircon (Fig. 4a) show agreement along [0 01 1] and [1 000$]$ (through IS2), greater than $50 \mathrm{~kJ} / \mathrm{mol}$ difference in [101], and IS3 was not considered in (Reich et al., 2007). Along [001] and [100] (through IS3), activation energies from DFT calculations are slightly higher than results from empirical potentials, suggesting empirical potentials do not consider some of the essential interactions between $\mathrm{He}$ and the atoms in the structure for diffusion. $E_{A}$ along [001] and [100] (through IS2) are similar (Table 1, Fig. 4a) because both of these interstitial sites have very similar nearest-neighbor distances.

Despite small variations due to computational differences, the four computational studies in zircon converge qualitatively to the same result (Fig. 4b): the energy barriers are lowest along [001] $(\sim 0-50 \mathrm{~kJ} / \mathrm{mol})$, slightly higher along [1 01$]$ ( $\sim 25-100 \mathrm{~kJ} / \mathrm{mol})$, and highest along [100] 
( $\sim 220-270 \mathrm{~kJ} / \mathrm{mol}$ ). Noticeably, $E_{A}$ is higher for apatite than zircon, regardless of the computational method used (Fig. 4b), resulting in smaller diffusivities. Currently there are no other computational studies in apatite for comparison.

\subsection{Comparison of computational results to experimental measurements}

Clear trends and agreements emerge when the calculations are compared to experimental measurements. Diffusivity in zircon is anisotropic with the highest values along [001] (Fig. 5), consistent with the presence of open channels along [0 011$]$ (Fig. 1b). Diffusivitiy in apatite is significantly less anisotropic than in zircon.

The largest differences between calculations and experiments are that calculations result in a much higher degree of anisotropy and significantly lower activation energies than experimental measurements. In addition, the calculations indicate diffusion in ideal zircon to be greater (faster) than in ideal apatite, contrary to experimental measurements where He diffusion in apatite is faster than in zircon (Fig. 5). In zircon, [001] (perpendicular to the page in Fig. 1b) has open channels; however, apatite's channels along [0 01 1] (perpendicular to the page in Fig. 1a) are less open, suggesting defect-free zircon has fewer structural barriers to diffusion than apatite and should have greater diffusivity. The calculated diffusivities in zircon along [0 011$]$ are greater than experimental measurements by over 5 orders of mangitude. Diffusion varies by $10-20$ orders of magnitude depending on direction, whereas the degree of anisotropy in experiments is only 2-3 orders of magnitude (Fig. 5b). The DFT method does not include the thermal motion of atoms. Vibrating lattice atoms may act to hinder diffusion along open channels and widen narrow openings along other channels, effectively decreasing the anisotropy of the diffusion rates, however the overall impact on transport properties should be small. The task for future work is to develop a force-field for empirical potential methods that replicates the physical properties of $\mathrm{He}$ in fluroapatite and the energetics of the He diffusion paths as derived from our quantum mechanical calculations. Then, in such an additional study, molecular dynamics simulations will quantify the influence of thermal vibrations on the reduction the anisotropy of diffusion coefficients.

The higher activation barriers in experimental zircon measurements are more likely explained by the presence of radiation damage in natural samples. The differences between calculations and experiments along [00 1] suggest natural zircon may have barriers to the mobility of He that block some of the open channels in the ideal structure, a claim also suggested by Farley (2007). Zircon often has domains of radiation damage that accumulate as long the temperature remains below the annealing temperature (Weber et al., 1997; Ewing, 2001). This damage will create interstitial barriers that can inhibit mobility of He along the [0 01 l] channel and decrease anisotropy by removing barriers in [100]. A recent computational study found that the addition of simple point defects to zircon slows diffusion along $\left[\begin{array}{lll}0 & 0 & 1]\end{array}\right.$ and speeds diffusion along [100] (Saadoune and de
Leeuw, 2009). Natural samples have a more complicated defect structure than the simple defects considered in Saadoune and de Leeuw (2009), and additional studies are needed to fully characterize the role of radiation damage on diffusion.

Typically, radiation-induced transformations cause the change of an anisotropic material to an isotropic material (Ewing et al., 2000). This effect is most evident in the reduced birefringence as a function of increasing dose (Chakoumakos et al., 1987; Palenik et al., 2003). Radiation damage in zircon is remarkably persistent; in the absence of elevated temperatures, most damage is preserved (Lumpkin and Ewing, 1988). The radiation-damaged microstructure of natural zircons will vary widely depending on dose and extent of thermally-activated annealing (Ewing et al., 2000). It is plausible that natural zircon could have varying levels of damage accumulation at elevated temperature allowing cascades to recover or anneal causing fewer barriers along [001], yielding diffusivities as high as those shown in Fig. 5b.

The calculated values in Fig. 5 are the bounding diffusivity values for ideal, defect-free zircon. Values for measured diffusivity in zircon are between the calculated values along $[001]$ and [100] (Fig. 5b). If He were to travel around an area of radiation damage along [001], He would have to diffuse along [ 10101$]$ or [ 100$]$. The high energy barriers along [1 000$]$ make that path unlikely. Therefore, He may either travel around radiation damage, via [101], or through the radiation damage.

Radiation damage in apatite anneals at lower temperatures than in zircon. This has been well demonstrated by systematic studies of ion beam damage in apatite, monazite and zircon as a function of temperature (Fig. 8) (Weber et al., 1998; Ewing, 1999). For all three phases, apatite, monazite and zircon, the dose to cause damage or amorphization is approximately the same. The variations are small but caused by variations in atomic mass and the cross-section for the ion-solid interaction. The critical point is that the phases have very different recovery behaviors. Apatite and monazite recover their crystallinity at much lower temperatures. Zircon generally retains the damage because the recovery is slower and does not occur until one reaches higher temperatures. As a result, the damage that accumulates due to alpha-decay events is more easily annealed in apatite than in zircon; thus, apatite will have fewer remaining defects and a less damaged structure (Weber et al., 1997; Ewing, 2001). Because of the lower annealing temperature, apatite is described as being more "resistant" to radiation damage due to the efficiency of the recovery process (Chaumont et al., 2002). Because apatite will generally retain less radiation damage than zircon, the calculated diffusivities for ideal apatite are in better agreement with those measured for natural apatite. Still, a small amount of radiation damage must be present in natural apatite, and this will result in lower He diffusion rates that are more isotropic as compared with the calculated values. A recent study investigating the effect of radiation damage on He mobility found that as the amount of damage increased, the activation barriers and closure temperatures increased as well, hindering diffusion (Shuster and Farley, 2009). Models in 


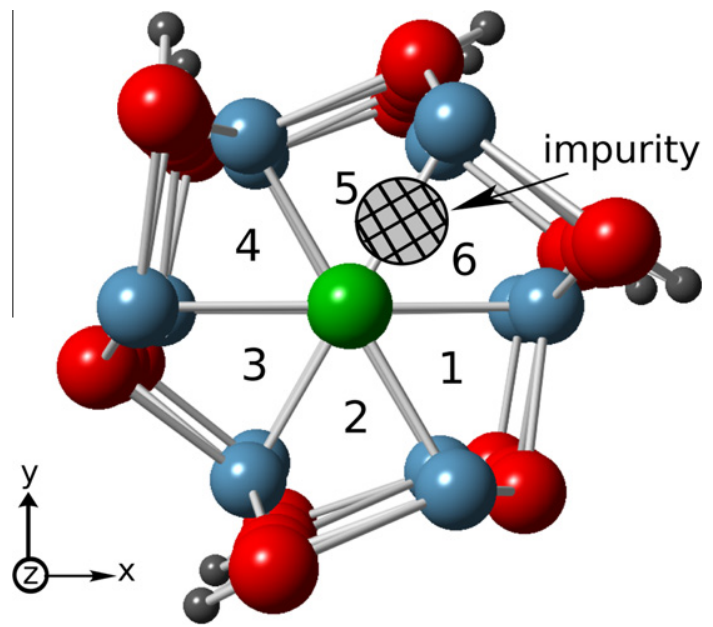

Fig. 6. Close-up of F-column in apatite. He diffusion along the [001] F column is structurally and energetically the same along channels 1 through 6 . A hypothetical impurity is shown along the [001] F-column (large gray circle) blocking channels 5 and 6 .

apatite have shown diffusion to be dependent on radiation damage (Flowers et al., 2009) and He loss to be related to annealing of radiation damage (Gautheron et al., 2009). The key to understanding He loss in natural samples thus depends on a clear knowledge of the damage microstructure, which will depend on the U- and Th-concentration, the age of the sample and its thermal history.

Further research is necessary to characterize the role of defects and impurities on He diffusion in apatite (analogous to zircon (Saadoune and de Leeuw, 2009)). We offer the following explanation for the observed and calculated diffusion mechanisms. A single impurity along the [0 011$]$ F-column may not hinder or significantly alter He diffusion. A hypothetical impurity is shown in Fig. 6. He traveling along channels 5 or 6 would be hindered by the impurity. However, the energy cost of He moving from column 6 to 1 or 5 to 4 would be minimal; He could switch to an unblocked channel and continue traveling unimpeded. For an impurity in apatite to significantly alter He diffusion rates, all six channels of the F column must be blocked. The substitution of a larger atom or group for $\mathrm{F}$ (such as $\mathrm{Cl}$, hydroxide, or carbonate groups) could reduce the interstitial space for $\mathrm{He}$ in all channels on the $[001] \mathrm{F}$ column and decrease the $\mathrm{He}$ diffusion rate.

In general, impurities along any channel will hinder $\mathrm{He}$ transport, while vacancies will speed up He transport. For rigorous simulations on the impact of defects on diffusion, the apatite defect structure, variations of the defect structure from sample to sample, and the size of the radiation damaged areas will all need to be included in the calculations.

\subsection{Calculation of closure temperatures}

The closure temperature $\left(T_{c}\right)$ can be used to constrain the age of a mineral. Above the closure temperature, daughter products of radioactive decay are lost from the mineral; below the closure temperature they are retained because the diffusion rate is so low that they are not released (Dodson, 1973). The ability of a mineral to retain
He depends on the kinetic barriers $\left(E_{A}, D_{0}\right)$ within the mineral, the cooling rate, and the size and shape of the grain. The closure temperature can be calculated from the Dodson equation (Dodson, 1973; Cherniak et al., 2009)

$T_{c}=\frac{E_{A} / R}{\ln \left(\frac{A R T_{c}^{2} D_{0}}{a^{2} E_{A} \mathrm{~d} T / \mathrm{d} t}\right)}$

where $E_{A}$ (activation energy barrier) and $\mathrm{D}_{0}$ (diffusion prefactor) are from the calculations in this study (Table 1) and $R$ is the gas constant. A cooling rate $(\mathrm{d} T / \mathrm{d} t)$ of $10^{\circ} \mathrm{C} / \mathrm{Ma}$ $\left(3.16888 \times 10^{-13} \mathrm{~K} / \mathrm{s}\right)$ was used (consistent with (Cherniak, 2000)). The correct geometric parameter $(A)$ for anisotropic systems is not known, therefore geometric parameters (Dodson, 1973) of 55 (sphere, upper bound) and 8.7 (planar sheet, lower bound) were both used to provide bounding values. If two grains have exactly the same properties, but one grain is larger than the other, $T_{c}$ of $\mathrm{He}$ in the larger grain will be higher because $\mathrm{He}$ has further to diffuse in order to escape the grain. Since grain sizes can vary, $T_{c}$ is plotted as a function of size, $a$, in Fig. 7. If a mineral has a high activation barrier (i.e., low diffusion constants or rates), higher energy is required for He to overcome the energy barrier, and $T_{c}$ will be higher. Conversely, low energy activation barriers (i.e., higher diffusion constants or rates) have a lower $T_{c}$ because less energy is required to overcome the energy barrier. Typical values for $T_{c}$ are $100-200{ }^{\circ} \mathrm{C}$ for zircon and $55-75^{\circ} \mathrm{C}$ for apatite (Cherniak et al., 2009).

At $A=55$ and $a=10^{-4}, T_{c}$ in ideal apatite is $-35^{\circ} \mathrm{C}$ $\left(10^{\circ} \mathrm{C}\right)$ in $\left[\begin{array}{lll}0 & 0 & 1\end{array}\right]\left(\left[\begin{array}{ll}1 & 10\end{array}\right]\right)$. In ideal zircon, $T_{c}$ is $-150^{\circ} \mathrm{C}$ $\left(380^{\circ} \mathrm{C}\right)$ in $[001]([100])$. In both apatite and zircon, $\left[\begin{array}{lll}0 & 0 & 1\end{array}\right]$ has the lowest closure temperature (Fig. 7). Changing

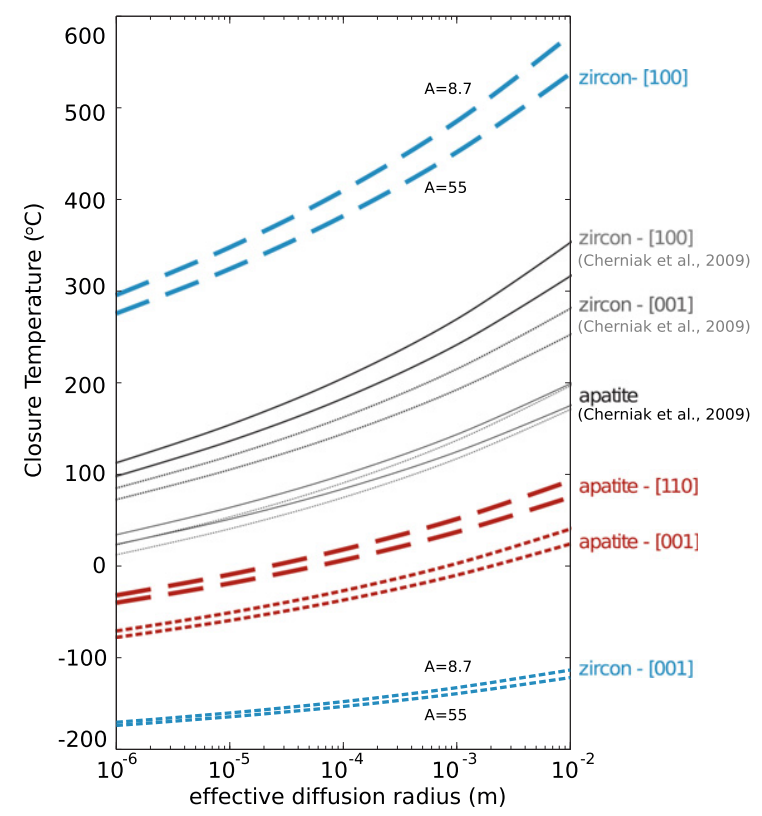

Fig. 7. Calculated closure temperatures, $T_{\mathrm{c}}$, as a function of effective diffusion radius, $a$, plotted in comparison with results from experimental ion beam measurements (Cherniak et al., 2009). Closure temperatures are plotted for both assuming planar geometry (upper bound, $A=8.7$ ) and spherical geometry (lower bound, $A=55$ ) of the crystals. 


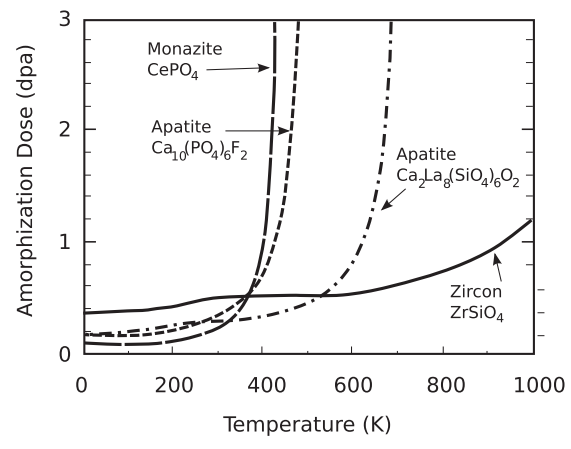

Fig. 8. Amorphization as a function of temperature in apatite, monazite and zircon. Results, obtained from ion irradiation, are reproduced from Fig. 18 of Weber et al. (1998). Plot illustrates the critical temperatures, the temperature at which damage anneals and no damage accumulates, for each mineral.

the geometric parameter for a given crystallographic direction will change $T_{c}$ by at most $30{ }^{\circ} \mathrm{C}$. The calculated $T_{c}$ in zircon is significantly lower in zircon than in apatite, due to the larger diffusivities in ideal zircon as compared with apatite. Conversely, in experimental measurements, $T_{c}$ is higher in zircon than apatite.

The extremely low closure temperatures that were calculated are due to the relatively low energy barriers. In ideal zircon (calculation), $\mathrm{He}$ is retained between two relatively low activation energy barriers, and the resulting $T_{c}$ values are very low. In natural zircon, He would be trapped between two defects, e.g., due to radiation damage, requiring a higher temperature to cause the release of the He. In a recent study, $T_{\mathrm{c}}$ was measured experimentally as a function of increasing radiation damage, and it was found that $T_{\mathrm{c}}$ increases with increasing damage (Shuster and Farley, 2009). Research on synthetic zircon samples with limited radiation damage also found the synthetic zircon structure has a significantly lower $T_{\mathrm{c}}$ than natural zircon structures (Farley, 2007). Both support the hypothesis that an undamaged (ideal) structure would have a lower closure temperature than those observed in natural samples. Interestingly, closure temperatures for synthetic zircon (Farley, 2007) are lower than in natural samples but still significantly higher than $T_{\mathrm{c}}$ of ideal zircon in the calculations (Fig. 7). Synthetic samples should have less radiation damage than natural samples, the difference between synthetic and ideal zircon could possibly also be due to crystallographic defects, impurity substitutions, and lattice distortions.

\section{CONCLUSIONS}

In an ideal structure in which no defects are present, such as those used in the DFT calculations: (i) the diffusivity of $\mathrm{He}$ is greater in zircon than apatite; (ii) the anisotropy of the diffusivity is greater in zircon than apatite; (iii) zircon has a lower closure temperature than apatite. Calculations and measurements show better agreement for apatite than zircon, consistent with experimental results that show that zircon retains its radiation-induced microstructure to higher temperatures. Under most conditions, apatite will retain less radiation damage than zircon.
The differences between the ideal and natural zircon and apatite suggest that the diffusion kinetics and closure depend on the radiation damage microstructure. Individual recoil cascades are generally $5 \mathrm{~nm}$ in diameter and can easily result in interstitial defects that block fast paths for diffusion. However, at very high doses, where recoil cascades overlap, bulk volume changes may be large for zircon $(16 \%)$, and the He diffusivity may increase. Thus, the wide variations in thermochronologic determinations are probably due to the different damage structures that result from the complex interactions between accumulation damage dose and thermal annealing.

The purpose of this study was not to match computational with experimental values; rather, computational simulations were used to understand the role of structure on variations in diffusivity in different structure-types and along different crystallographic directions. Clear differences in diffusivity of He for computed structures and natural samples suggest natural samples are far from ideal in their structure, probably the result of varying degrees of radiation damage.

\section{ACKNOWLEDGEMENTS}

A.B. acknowledges support from the Turner Postdoctoral Fellowship at the University of Michigan and from the Office of Basic Energy Sciences (DE-FG02-973445656). UB and the development of diffusion theory were supported by the DOE/BES grant DEFG02-06ER15783. Jishnu Bhattacharya and Julie Tucker provided guidance on the use of the nudged elastic band method for diffusion calculations. We are grateful to Dane Morgan for the use of the scripting suite, convasp (Curtarolo et al., 2005), and for previous computational diffusion discussions. Computational support was provided by Peter van Keken and Michael Messina through the legato cluster at the University of Michigan. Reviews from Peter Reiners, Richard Ketcham and two anonymous reviewers were much appreciated.

\section{REFERENCES}

Allnatt A. R. and Lidiard A. B. (1993) Atomic Transport in Solids. Cambridge University Press, Cambridge.

Ammann M. W., Brodholt J. P. and Dobson D. P. (2010) Simulating diffusion. In BookSimulating Diffusion (eds. R. Wentzcovitch and L. Stixrude). Mineralogical Soc. Amer., Chantilly, pp. 201-224.

Chakoumakos B. C., Murakami T., Lumpkin G. R. and Ewing R. C. (1987) Alpha-decay - induced fracturing in zircon: the transition from the crystalline to the metamict state. Science 236, 1556-1559.

Chaumont J., Soulet S., Krupa J. C. and Carpena J. (2002) Competition between disorder creation and annealing in fluoroapatite nuclear waste forms. J. Nucl. Mater. 301, 122128.

Cherniak D. J. (2000) Rare earth element diffusion in apatite. Geochim. Cosmochim. Acta 64, 3871-3885.

Cherniak D. J., Watson E. B. and Thomas J. B. (2009) Diffusion of helium in zircon and apatite. Chem. Geol. 268, 155-166.

Curtarolo S., Morgan D. and Ceder G. (2005) Accuracy of ab initio methods in predicting the crystal structures of metals: a review of 80 binary alloys. Calphad 29, 163-211.

Dodson M. H. (1973) Closure temperature in cooling geochronological and petrological systems. Contrib. Mineral. Petrol. 40, 259-274. 
Ehlers T. A. (2005) Crustal thermal processes and the interpretation of thermochronometer data. In Low-Temperature Thermochronology: Techniques, Interpretations, and Applications. (eds. P. W. Reiners, T. A. Ehlers). Rev. Mineral. Geochem. 58, Mineralogical Society of America, pp. 315-350.

Ehlers T. A. and Farley K. A. (2003) Apatite (U-Th)/He thermochronometry: methods and applications to problems in tectonic and surface processes. Earth Planet. Sci. Lett. 206, 1-14.

Ewing R. C. (1999) Nuclear waste forms for actinides. Proc. Nat. Acad. Sci. U.S.A. 96, 3432-3439.

Ewing R. C. (2001) The design and evaluation of nuclear-waste forms: clues from mineralogy. Can. Mineral. 39, 697-715.

Ewing R. C., Meldrum A., Wang L. M. and Wang S. X. (2000) Radiation-induced amorphization. In Transformation Processes in Minerals. (eds. S. A. T. Redfern, M. A. Carpenter). Rev. Mineral. Geochem. 39, Mineralogical Society of America, pp. 319-361.

Farley K. A. (2000) Helium diffusion from apatite: general behavior as illustrated by Durango fluorapatite. J. Geophys. Res. Solid Earth 105, 2903-2914.

Farley K. A. (2007) He diffusion systematics in minerals: evidence from synthetic monazite and zircon structure phosphates. Geochim. Cosmochim. Acta 71, 4015-4024.

Flowers R. M., Ketcham R. A., Shuster D. L. and Farley K. A. (2009) Apatite (U-Th)/He thermochronometry using a radiation damage accumulation and annealing model. Geochim. Cosmochim. Acta 73, 2347-2365.

Gautheron C., Tassan-Got L., Barbarand J. and Pagel M. (2009) Effect of alpha-damage annealing on apatite (U-Th)/He thermochronology. Chem. Geol. 266, 157-170.

Gomer R. (1990) Diffusion of adsorbates on metal-surfaces. Rep. Prog. Phys. 53, 917-1002.

Hafner J., Wolverton C. and Ceder G. (2006) Toward computational materials design: the impact of density functional theory on materials research. MRS Bull. 31, 659-665.

Huber C., Cassata W. S. and Renne P. R. (2011) A lattice Boltzmann model for noble gas diffusion in solids: the importance of domain shape and diffusive anisotropy and implications for thermochronometry. Geochim. Cosmochim. Acta 75, 2170-2186.

Jónsson H., Mills G. and Jacobsen K. W. (1998) Nudged elastic band method for finding minimum energy paths of transitions. In Classical and Quantum Dynamics in Condensed Phase Simulations (eds. B. J. Berne, G. Ciccotti and D. F. Coker). World Scientific, pp. 385-404, <http://www.worldscientific.com/index.html $>$.

Kresse G. and Furthmuller J. (1996a) Efficiency of ab-initio total energy calculations for metals and semiconductors using a plane-wave basis set. Comput. Mater. Sci. 6, 15-50.

Kresse G. and Furthmuller J. (1996b) Efficient iterative schemes for ab initio total-energy calculations using a plane-wave basis set. Phys. Rev. B 54, 11169-11186.

Kresse G. and Hafner J. (1993) Abinitio molecular-dynamics for liquid-metals. Phys. Rev. B 47, 558-561.

Kutner R. (1981) Chemical diffusion in the lattice gas of noninteracting particles. Phys. Lett. A 81, 239-240.

Lumpkin G. R. and Ewing R. C. (1988) Alpha-decay damage in minerals of the pyrochlore group. Phys. Chem. Miner. 16, 2-20.

Mills G., Jonsson H. and Schenter G. K. (1995) Reversible work transition-state theory - application to dissociative adsorption of hydrogen. Surf. Sci. 324, 305-337.

Palenik C. S., Nasdala L. and Ewing R. C. (2003) Radiation damage in zircon. Am. Mineral. 88, 770-781.

Perdew J. P., Burke K. and Ernzerhof M. (1996) Generalized gradient approximation made simple. Phys. Rev. Lett. 77, 38653868 .
Reich M., Ewing R. C., Ehlers T. A. and Becker U. (2007) Lowtemperature anisotropic diffusion of helium in zircon: implications for zircon (U-Th)/He thermochronometry. Geochim. Cosmochim. Acta 71, 3119-3130.

Reiners P. W. and Shuster D. L. (2009) Thermochronology and landscape evolution. Phys. Today 62, 31-36.

Reiners P. W., Ehlers T. A. and Zeitler P. K. (2005) Past, present, and future of thermochronology. In Low-Temperature Thermochronology: Techniques, Interpretations, and Applications (eds. P. W. Reiners, T. A. Ehlers). Rev. Mineral. Geochem. 58, Mineralogical Society of America, pp. 1-18. .

Saadoune I. and de Leeuw N. H. (2009) A computer simulation study of the accommodation and diffusion of He in uraniumand plutonium-doped zircon ( $\mathrm{ZrSiO} 4)$. Geochim. Cosmochim. Acta 73, 3880-3893.

Saadoune I., Purton J. A. and de Leeuw N. H. (2009) He incorporation and diffusion pathways in pure and defective zircon $\mathrm{ZrSiO} 4$ : a density functional theory study. Chem. Geol. 258, 182-196.

Sholl D. and Steckel J. (2009) Density Functional Theory: A Practical Introduction. John Wiley \& Sons, Inc., Hoboken, New Jersey.

Shuster D. L. and Farley K. A. (2005) He-4/He-3 thermochronometry: Theory, practice, and potential complications. In Low-Temperature Thermochronology: Techniques, Interpretations, and Applications (eds. P.W. Reiners, T. A. Ehlers). Rev. Mineral. Geochem. 58, Mineralogical Society of America, pp. 181-203.

Shuster D. L. and Farley K. A. (2009) The influence of artificial radiation damage and thermal annealing on helium diffusion kinetics in apatite. Geochim. Cosmochim. Acta 73, 183-196.

Thomson S. N., Brandon M. T., Tomkin J. H., Reiners P. W., Vasquez C. and Wilson N. J. (2010) Glaciation as a destructive and constructive control on mountain building. Nature 467 , 313-317.

Van der Ven A. and Ceder G. (2005) First principles calculation of the interdiffusion coefficient in binary alloys. Phys. Rev. Lett. 94.

Van der Ven A., Ceder G., Asta M. and Tepesch P. D. (2001) Firstprinciples theory of ionic diffusion with nondilute carriers. Phys. Rev. B 64, 184307-1-184307-17.

Van der Ven A., Thomas J. C., Xu Q. C., Swoboda B. and Morgan D. (2008) Nondilute diffusion from first principles: Li diffusion in LixTiS2. Phys. Rev. B 78, 104306.

Vineyard G. (1957) Frequency factors and isotope effects in solid state rate processes. J. Phys. Chem. Solids 3, 121-127.

Watson E. B. and Baxter E. F. (2007) Diffusion in solid-Earth systems. Earth Planet. Sci. Lett. 253, 307-327.

Weber W. J., Ewing R. C. and Meldrum A. (1997) The kinetics of alpha-decay-induced amorphization in zircon and apatite containing weapons-grade plutonium or other actinides. $J$. Nucl. Mater. 250, 147-155.

Weber W. J., Ewing R. C., Catlow C. R. A., de la Rubia T. D., Hobbs L. W., Kinoshita C., Matzke H., Motta A. T., Nastasi M., Salje E. K. H., Vance E. R. and Zinkle S. J. (1998) Radiation effects in crystalline ceramics for the immobilization of high-level nuclear waste and plutonium. J. Mater. Res. 13, 1434-1484.

Wolf R. A., Farley K. A. and Silver L. T. (1996) Helium diffusion and low-temperature thermochronometry of apatite. Geochim. Cosmochim. Acta 60, 4231-4240.

Associate editor: Peter Reiners 


\section{SciVerse ScienceDirect}

Geochimica et Cosmochimica Acta xxx (2012) xxx-xxx

\section{Geochimica et \\ Cosmochimica \\ Acta}

www.elsevier.com/locate/gca

\section{Corrigendum}

\section{Corrigendum to "He diffusion and closure temperatures in apatite and zircon: A density functional theory investigation" [Geochim. Cosmochim. Acta 86 (2012) 228-238]}

The authors regret the grant information in the second sentence of the acknowledgements section is incorrect. The corrected sentence appears below within the full text of the acknowledgement paragraph:

A.B. acknowledges support from the Turner Postdoctoral Fellowship at the University of Michigan and from the Office of Basic Energy Sciences (DE FG02 97 ER45656). UB and the development of diffusion theory were supported by the DOE/NEUP Grant DE AC07 05 ID14517. Jishnu Bhattacharya and Julie Tucker provided guidance on the use of the nudged elastic band method for diffusion calculations. We are grateful to Dane Morgan for the use of the scripting suite, convasp (Curtarolo et al., 2005), and for previous computational diffusion discussions. Computational support was provided by Peter van Keken and Michael Messina through the legato cluster at the University of Michigan. Reviews from Peter Reiners, Richard Ketcham and two anonymous reviewers were much appreciated.

The authors would like to apologise for any inconvenience caused.

Amelia Bengtson, Rodney C. Ewing, Udo Becker Department of Earth and Environmental Sciences,

University of Michigan, Ann Arbor, MI 48109-1005,

United States

DOI of original article: 10.1016/j.gca.2012.03.004. 